\title{
No Difference in Orbital Parameters of RV-detected Giant Planets between 0.1 and 5 au in Single versus Multi-stellar Systems
}

\author{
Henry $\mathrm{Ngo}^{1}$, Heather A. Knutson ${ }^{1}$, Marta L. Bryan ${ }^{2}$, Sarah Blunt ${ }^{3,4,5}$, Eric L. Nielsen ${ }^{3,5}$, Konstantin Batygin ${ }^{1}$, \\ Brendan P. Bowler ${ }^{6,10}$, Justin R. Crepp ${ }^{7}$, Sasha Hinkley ${ }^{8}$, Andrew W. Howard ${ }^{2}$, and Dimitri Mawet ${ }^{2,9}$ \\ ${ }^{1}$ Division of Geological and Planetary Sciences, California Institute of Technology, Pasadena, CA, USA; hngo@caltech.edu \\ ${ }^{2}$ Department of Astronomy, California Institute of Technology, Pasadena, CA, USA \\ ${ }^{3}$ SETI Institute, Carl Sagan Center, Mountain View, CA, USA \\ ${ }^{4}$ Department of Physics, Brown University, Providence, RI, USA \\ ${ }^{5}$ Kavli Institute for Particle Astrophysics and Cosmology, Stanford University, Stanford, CA, USA \\ ${ }^{6}$ McDonald Observatory and the Department of Astronomy, The University of Texas at Austin, Austin, TX, USA \\ ${ }^{7}$ Department of Physics, University of Notre Dame, Notre Dame, IN, USA \\ ${ }^{8}$ Department of Physics and Astronomy, University of Exeter, Exeter, UK \\ ${ }^{9}$ Jet Propulsion Laboratory, California Institute of Technology, Pasadena, CA, USA \\ Received 2017 February 11; revised 2017 April 5; accepted 2017 April 6; published 2017 May 4
}

\begin{abstract}
Our Keck/NIRC2 imaging survey searches for stellar companions around 144 systems with radial velocity (RV) detected giant planets to determine whether stellar binaries influence the planets' orbital parameters. This survey, the largest of its kind to date, finds eight confirmed binary systems and three confirmed triple systems. These include three new multi-stellar systems (HD 30856, HD 86081, and HD 207832) and three multi-stellar systems with newly confirmed common proper motion (HD 43691, HD 116029, and HD 164509). We combine these systems with seven RV planet-hosting multi-stellar systems from the literature in order to test for differences in the properties of planets with semimajor axes ranging between 0.1 and 5 au in single versus multi-stellar systems. We find no evidence that the presence or absence of stellar companions alters the distribution of planet properties in these systems. Although the observed stellar companions might influence the orbits of more distant planetary companions in these systems, our RV observations currently provide only weak constraints on the masses and orbital properties of planets beyond $5 \mathrm{au}$. In order to aid future efforts to characterize long-period RV companions in these systems, we publish our contrast curves for all 144 targets. Using four years of astrometry for six hierarchical triple star systems hosting giant planets, we fit the orbits of the stellar companions in order to characterize the orbital architecture in these systems. We find that the orbital plane of the secondary and tertiary companions are inconsistent with an edge-on orbit in four out of six cases.
\end{abstract}

Key words: binaries: close - binaries: eclipsing - methods: observational - planetary systems planets and satellites: dynamical evolution and stability - techniques: high angular resolution

Supporting material: machine-readable tables, tar.gz file

\section{Introduction}

Gas giant exoplanets have been found to orbit their host stars over a wide range of orbital separations, spanning more than four orders of magnitude from close-in "hot Jupiters" to distant directly imaged planetary mass companions (Fischer et al. 2014a; Bowler 2016). Conventional core accretion models (e.g., Pollack et al. 1996) have argued that giant planet formation is most favorable just beyond the water ice line, where the increased density of solids allows for the rapid formation of cores large enough to accrete a significant gas envelope. If correct, this would suggest that most short-period gas giant planets formed at intermediate separations and then migrated inward to their present-day locations (e.g., Lin et al. 1996). However, new modeling work motivated by the numerous close-in super-Earth exoplanetary systems (e.g., Fressin et al. 2013; Mulders et al. 2015) has suggested that it may be possible to form closein gas giant planets in situ, providing an alternative to the migration-driven hypothesis (Bodenheimer et al. 2000; Batygin et al. 2016; Boley et al. 2016). We note that the conglomeration of the rocky core itself is a separate process from the accretion of the gaseous envelope. In other words, local formation of the core,

\footnotetext{
${ }^{10}$ Hubble fellow.
}

followed by extended gas accretion as well as long range migration of the core followed by rapid gas accretion at close-in separations both represent viable in situ formation scenarios. It is unclear what role, if any, stellar companions might play in these processes. However, the fact that approximately $44 \%$ of field stars (Duquennoy \& Mayor 1991; Raghavan et al. 2010) are found in multiple star systems makes this a crucial question for studies of giant planet formation and/or migration.

Many recent imaging surveys have been carried out to determine the frequency of outer stellar companions in systems with close-in $(a<0.1 \mathrm{au})$ transiting giant planets (Ngo et al. 2015, 2016; Wang et al. 2015b; Wöllert \& Brandner 2015; Wöllert et al. 2015; Evans et al. 2016). However, there are relatively few studies that have examined the architectures of systems with intermediate separation (0.1-5 au) planets. Giant planets at these intermediate separations have a different migration history than their short-period counterparts. Some dynamical interactions depend strongly on orbital separations. For example, close-in planets are more tightly coupled to the host star and would therefore experience more rapid tidal circularization than planets on more distant orbits. In addition, the environment of the protoplanetary disk varies as a function of radial separation so these intermediate planets may be the 
Table 1

Summary of NIRC2 AO Observations

\begin{tabular}{|c|c|c|c|c|c|c|c|}
\hline Target & $N_{c c}$ & UT Obs. Date & Filter & Array & $T_{\text {int }}(\mathrm{s})$ & $N_{\text {fit }}$ & $N_{\text {stack }}$ \\
\hline \multicolumn{8}{|c|}{ RV planet host stars } \\
\hline GJ 317 & 0 & 2014 Nov 07 & $K_{c}$ & 1024 & 12.0 & $\cdots$ & 12 \\
\hline GJ 433 & 0 & 2015 Jan 09 & $K_{c}$ & 1024 & 13.6 & $\cdots$ & 12 \\
\hline GJ 667C & 0 & 2014 Jul 12 & $K_{c}$ & 1024 & 13.6 & $\cdots$ & 12 \\
\hline \multirow[t]{3}{*}{ GJ 876} & 0 & 2013 Aug 19 & $K_{c}$ & 512 & 10.6 & $\cdots$ & 12 \\
\hline & & 2013 Oct 17 & $J_{c}$ & 512 & 10.6 & $\cdots$ & $\ldots$ \\
\hline & & 2013 Oct 17 & $K_{c}$ & 512 & 10.6 & $\cdots$ & 12 \\
\hline HD 1461 & 0 & 2014 Jul 12 & $K_{c}$ & 512 & 15.9 & $\cdots$ & 12 \\
\hline HD 1502 & 0 & 2013 Aug 19 & $K_{c}$ & 1024 & 9.0 & $\cdots$ & 12 \\
\hline HD 3651 & 0 & 2013 Aug 19 & $K_{c}$ & 256 & 9.0 & $\cdots$ & 12 \\
\hline HD 4203 & 0 & 2013 Aug 19 & $K_{c}$ & 1024 & 9.0 & $\ldots$ & 12 \\
\hline HD 4208 & 0 & 2013 Nov 17 & $K_{c}$ & 1024 & 10.0 & $\cdots$ & 15 \\
\hline HD 4313 & 0 & 2013 Oct 17 & $K_{c}$ & 512 & 10.6 & $\ldots$ & 12 \\
\hline HD 5319 & 0 & 2014 Jul 12 & $K_{c}$ & 1024 & 13.6 & $\cdots$ & 12 \\
\hline \multirow[t]{3}{*}{ HD 5891} & 0 & 2013 Oct 17 & $J_{c}$ & 512 & 10.6 & $\ldots$ & $\ldots$ \\
\hline & & 2013 Oct 17 & $K_{c}$ & 512 & 10.6 & $\ldots$ & 12 \\
\hline & & 2015 Dec 19 & $K_{s}$ & 1024 & 15.0 & $\cdots$ & 12 \\
\hline HD 7924 & 0 & 2014 Oct 03 & $K_{c}$ & 1024 & 13.6 & $\ldots$ & 12 \\
\hline HD 8574 & 0 & 2015 Jan 09 & $K_{c}$ & 1024 & 13.6 & $\cdots$ & 12 \\
\hline HD 10697 & 0 & 2015 Dec 19 & $K_{c}$ & 1024 & 15.0 & $\ldots$ & $\ldots$ \\
\hline HD 11506 & 0 & 2013 Nov 17 & $K_{c}$ & 1024 & 10.0 & $\cdots$ & 15 \\
\hline HD 11964A & 0 & 2013 Nov 17 & $K_{c}$ & 512 & 10.6 & $\cdots$ & 12 \\
\hline HD 12661 & 0 & 2013 Aug 19 & $K_{c}$ & 1024 & 9.0 & $\cdots$ & 12 \\
\hline HD 13931 & 0 & 2015 Jan 09 & $K_{c}$ & 1024 & 13.6 & $\cdots$ & 12 \\
\hline HIP 14810 & 0 & 2013 Oct 17 & $K_{c}$ & 1024 & 9.0 & $\ldots$ & 10 \\
\hline HD 16141 & 0 & 2013 Aug 19 & $K_{c}$ & 512 & 10.6 & $\ldots$ & 12 \\
\hline HD 17156 & 0 & 2014 Oct 03 & $K_{c}$ & 1024 & 15.0 & $\ldots$ & 12 \\
\hline HD 22049 & 0 & 2016 Sep 13 & $K_{c}$ & 512 & 15.9 & $\cdots$ & 12 \\
\hline HIP 22627 & 0 & 2015 Jan 10 & $K_{c}$ & 1024 & 15.0 & $\ldots$ & 12 \\
\hline HD 24040 & 0 & 2015 Jan 10 & $K_{c}$ & 1024 & 13.6 & $\cdots$ & 12 \\
\hline HD 28678 & 0 & 2014 Oct 04 & $K_{c}$ & 1024 & 13.6 & $\cdots$ & 12 \\
\hline \multirow[t]{5}{*}{ HD 30856} & 1 & 2014 Oct 04 & $J_{c}$ & 1024 & 13.6 & 12 & $\cdots$ \\
\hline & & 2014 Oct 04 & $K_{c}$ & 1024 & 13.6 & 12 & 12 \\
\hline & & 2014 Dec 07 & $J_{c}$ & 1024 & 15.0 & 11 & $\ldots$ \\
\hline & & 2014 Dec 07 & $K_{c}$ & 1024 & 13.6 & 12 & 12 \\
\hline & & 2015 Oct 26 & $K_{c}$ & 1024 & 15.0 & 12 & 12 \\
\hline HD 32963 & 0 & 2014 Oct 04 & $K_{c}$ & 1024 & 13.6 & $\ldots$ & 12 \\
\hline HD 33142 & 0 & 2014 Oct 04 & $K_{c}$ & 1024 & 13.6 & $\cdots$ & 12 \\
\hline HD 33283 & 0 & 2014 Oct 04 & $K_{c}$ & 1024 & 12.5 & $\cdots$ & 12 \\
\hline HD 34445 & 0 & 2014 Nov 07 & $K_{c}$ & 1024 & 13.6 & $\ldots$ & 12 \\
\hline \multirow[t]{2}{*}{ HD 37124} & 0 & 2014 Jan 12 & $K^{\prime}$ & 1024 & 10.0 & $\ldots$ & 10 \\
\hline & & 2014 Dec 07 & $K_{c}$ & 1024 & 15.0 & $\ldots$ & 12 \\
\hline \multirow[t]{2}{*}{ HD 37605} & 0 & 2014 Jan 12 & $K^{\prime}$ & 1024 & 9.0 & $\ldots$ & 12 \\
\hline & & 2014 Dec 07 & $K_{c}$ & 1024 & 12.5 & $\cdots$ & 12 \\
\hline HD 38529 & 0 & 2015 Jan 10 & $K_{c}$ & 512 & 13.2 & $\ldots$ & 12 \\
\hline \multirow[t]{3}{*}{ HD 38801} & 0 & 2014 Jan 12 & $K^{\prime}$ & 1024 & 9.0 & $\ldots$ & 9 \\
\hline & & 2014 Jan 12 & $K_{c}$ & 1024 & 10.0 & $\cdots$ & 11 \\
\hline & & 2014 Dec 07 & $K_{c}$ & 1024 & 12.5 & $\cdots$ & 12 \\
\hline HD 40979 & 0 & 2014 Nov 10 & $K_{c}$ & 512 & 12.0 & $\ldots$ & 12 \\
\hline \multirow[t]{5}{*}{ HD 43691} & 2 & 2013 Dec 18 & $K_{c}$ & 1024 & 10.0 & 4 & 12 \\
\hline & & 2014 Dec 04 & $K_{c}$ & 1024 & 10.0 & 10 & 12 \\
\hline & & 2014 Dec 04 & $J_{c}$ & 1024 & 10.0 & 5 & $\ldots$ \\
\hline & & 2015 Oct 26 & $K_{c}$ & 1024 & 25.0 & 12 & 12 \\
\hline & & 2016 Sep 13 & $K_{c}$ & 1024 & 45.0 & 12 & 12 \\
\hline HD 45350 & 0 & 2013 Oct 17 & $K_{c}$ & 1024 & 9.0 & $\cdots$ & 12 \\
\hline HD 46375 & 0 & 2013 Oct 17 & $K_{c}$ & 1024 & 9.0 & $\cdots$ & 12 \\
\hline HD 49674 & 0 & 2013 Oct 17 & $K_{c}$ & 1024 & 9.0 & $\cdots$ & 12 \\
\hline HD 50499 & 0 & 2014 Nov 07 & $K_{c}$ & 1024 & 13.6 & $\cdots$ & 12 \\
\hline HD 50554 & 0 & 2013 Dec 18 & $K_{c}$ & 1024 & 10.0 & $\ldots$ & 12 \\
\hline HD 52265 & 0 & 2013 Oct 17 & $K_{c}$ & 512 & 10.6 & $\cdots$ & 12 \\
\hline HIP 57050 & 0 & 2014 May 21 & $K_{c}$ & 1024 & 12.5 & $\ldots$ & 12 \\
\hline HIP 57274 & 0 & 2014 May 21 & $K_{c}$ & 1024 & 12.5 & $\cdots$ & 12 \\
\hline
\end{tabular}


Table 1

(Continued)

\begin{tabular}{|c|c|c|c|c|c|c|c|}
\hline Target & $N_{c c}$ & UT Obs. Date & Filter & Array & $T_{\text {int }}(\mathrm{s})$ & $N_{\text {fit }}$ & $N_{\text {stack }}$ \\
\hline & & 2015 Dec 20 & $K_{c}$ & 1024 & 15.0 & $\ldots$ & 12 \\
\hline HD 66428 & 0 & 2013 Dec 18 & $K_{c}$ & 1024 & 10.0 & $\cdots$ & 12 \\
\hline HD 68988 & 0 & 2013 Dec 18 & $K_{c}$ & 1024 & 10.0 & $\ldots$ & 12 \\
\hline \multirow[t]{2}{*}{ HD 69830} & 0 & 2014 Jan 12 & $K_{c}$ & 1024 & 9.0 & $\cdots$ & 8 \\
\hline & & 2014 Dec 05 & $K_{c}$ & 512 & 13.2 & $\ldots$ & 12 \\
\hline \multirow[t]{2}{*}{ HD 72659} & 0 & 2014 Jan 12 & $K_{c}$ & 1024 & 9.0 & $\cdots$ & 15 \\
\hline & & 2014 Nov 10 & $K_{c}$ & 1024 & 13.6 & $\cdots$ & 12 \\
\hline HD 73256 & 0 & 2014 Nov 10 & $K_{c}$ & 1024 & 12.5 & $\cdots$ & 12 \\
\hline \multirow[t]{2}{*}{ HD 73534} & 0 & 2014 Jan 12 & $K_{c}$ & 1024 & 9.0 & $\cdots$ & 15 \\
\hline & & 2014 Dec 05 & $K_{c}$ & 1024 & 13.6 & $\ldots$ & 12 \\
\hline HD 74156 & 0 & 2014 Nov 07 & $K_{c}$ & 1024 & 13.6 & $\cdots$ & 12 \\
\hline HIP 74995 & 0 & 2013 Jul 04 & $K_{c}$ & 1024 & 9.0 & $\ldots$ & 12 \\
\hline HD 75732 & 0 & 2014 May 21 & $K_{c}$ & 256 & 10.0 & $\cdots$ & 12 \\
\hline \multirow[t]{3}{*}{ HD 75898} & 0 & 2014 May 21 & $J_{c}$ & 1024 & 12.5 & $\cdots$ & 12 \\
\hline & & 2014 May 21 & $K_{c}$ & 1024 & 12.5 & $\cdots$ & 12 \\
\hline & & 2015 Dec 20 & $K_{c}$ & 1024 & 15.0 & $\cdots$ & 12 \\
\hline HIP 79431 & 0 & 2013 Jul 04 & $K_{c}$ & 1024 & 9.0 & $\cdots$ & 12 \\
\hline \multirow[t]{2}{*}{ HD 80606} & 0 & 2014 Jan 12 & $K_{c}$ & 1024 & 10.0 & $\cdots$ & 12 \\
\hline & & 2014 Dec 07 & $K_{c}$ & 1024 & 15.0 & $\ldots$ & 12 \\
\hline \multirow[t]{2}{*}{ HD 82886} & 0 & 2014 Jan 12 & $K_{c}$ & 1024 & 9.0 & $\cdots$ & 15 \\
\hline & & 2014 May 21 & $K_{c}$ & 512 & 13.2 & $\ldots$ & 12 \\
\hline \multirow[t]{2}{*}{ HD 82943} & 0 & 2015 Jan 10 & $K_{c}$ & 1024 & 15.0 & $\cdots$ & 12 \\
\hline & & 2016 Jan 25 & $K_{c}$ & 1024 & 15.0 & $\cdots$ & 12 \\
\hline HIP 83043 & 0 & 2013 Jul 04 & $K_{c}$ & 1024 & 9.0 & $\cdots$ & 12 \\
\hline HD 83443 & 0 & 2015 Jan 09 & $K_{c}$ & 1024 & 12.5 & $\ldots$ & 12 \\
\hline \multirow[t]{2}{*}{ HD 86081} & 1 & 2013 Dec 18 & $K_{c}$ & 1024 & 10.0 & 9 & 12 \\
\hline & & 2014 Dec 05 & $K_{c}$ & 1024 & 12.0 & 10 & 12 \\
\hline HD 87883 & 0 & 2015 Jan 10 & $K_{c}$ & 1024 & 13.6 & $\cdots$ & 12 \\
\hline HD 88133 & 0 & 2013 Dec 18 & $K_{c}$ & 1024 & 10.0 & $\cdots$ & 12 \\
\hline \multirow{3}{*}{ HD 90043} & 0 & 2014 Jan 12 & $K_{c}$ & 512 & 10.6 & $\cdots$ & 12 \\
\hline & & 2014 May 21 & $K_{c}$ & 512 & 13.2 & $\cdots$ & 12 \\
\hline & & 2014 May 21 & $J_{c}$ & 256 & 13.5 & $\cdots$ & 6 \\
\hline HD 92788 & 0 & 2014 Dec 05 & $K_{c}$ & 1024 & 13.6 & $\cdots$ & 12 \\
\hline HD 95089 & 0 & 2014 Dec 05 & $K_{c}$ & 1024 & 13.6 & $\cdots$ & 12 \\
\hline HD 95128 & 0 & 2014 May 21 & $K_{c}$ & 256 & 15.0 & $\cdots$ & 12 \\
\hline HD 96063 & 0 & 2014 May 21 & $K_{c}$ & 1024 & 12.5 & $\cdots$ & 12 \\
\hline \multirow[t]{5}{*}{ HD 96167} & 1 & 2014 Jan 12 & $K_{c}$ & 1024 & 10.0 & 4 & 12 \\
\hline & & 2014 Dec 07 & $J_{c}$ & 1024 & 15.0 & 12 & $\cdots$ \\
\hline & & 2014 Dec 07 & $K_{c}$ & 1024 & 15.0 & 12 & 12 \\
\hline & & 2015 Jan 09 & $J_{c}$ & 1024 & 13.6 & 12 & $\cdots$ \\
\hline & & 2015 Jan 09 & $K_{c}$ & 1024 & 15.0 & 12 & 12 \\
\hline HD 97658 & 0 & 2013 Dec 18 & $K_{c}$ & 1024 & 9.0 & $\cdots$ & 12 \\
\hline \multirow[t]{2}{*}{ HD 99109} & 0 & 2014 May 21 & $K_{s}$ & 1024 & 18.1 & $\cdots$ & 12 \\
\hline & & 2016 Jan 25 & $K_{c}$ & 1024 & 15.0 & $\cdots$ & 12 \\
\hline HD 99492 & 0 & 2014 May 21 & $K_{c}$ & 512 & 13.2 & $\cdots$ & 12 \\
\hline HD 99706 & 0 & 2014 May 21 & $K_{c}$ & 512 & 13.2 & $\cdots$ & 12 \\
\hline HD 102195 & 0 & 2014 May 21 & $K_{c}$ & 1024 & 12.5 & $\cdots$ & 12 \\
\hline HD 102329 & 0 & 2014 May 21 & $K_{c}$ & 1024 & 13.6 & $\cdots$ & 12 \\
\hline HD 102956 & 0 & 2014 May 21 & $K_{c}$ & 1024 & 12.5 & $\cdots$ & 12 \\
\hline \multirow[t]{2}{*}{ BD-103166 } & 0 & 2014 Jan 12 & $K_{c}$ & 1024 & 20.0 & $\cdots$ & 12 \\
\hline & & 2015 Jan 09 & $K_{c}$ & 1024 & 15.0 & $\cdots$ & 12 \\
\hline HD 104067 & 0 & 2014 May 21 & $K_{c}$ & 1024 & 12.0 & $\cdots$ & 12 \\
\hline HD 106270 & 0 & 2014 May 21 & $K_{c}$ & 1024 & 12.5 & $\cdots$ & 12 \\
\hline HD 107148 & 0 & 2014 May 21 & $K_{c}$ & 1024 & 12.5 & $\ldots$ & 12 \\
\hline HD 108863 & 0 & 2014 Jun 09 & $K_{c}$ & 1024 & 13.6 & $\cdots$ & 12 \\
\hline \multirow[t]{2}{*}{ HD 108874} & 0 & 2015 Jan 09 & $K_{c}$ & 1024 & 12.0 & $\cdots$ & 12 \\
\hline & & 2015 Dec 20 & $K_{c}$ & 1024 & 15.0 & $\cdots$ & 12 \\
\hline HIP 109388 & 0 & 2014 Nov 07 & $K_{c}$ & 1024 & 13.6 & $\ldots$ & 12 \\
\hline HD 109749 & 0 & 2014 Jun 09 & $K_{c}$ & 1024 & 12.5 & $\cdots$ & 12 \\
\hline HD 114729 & 0 & 2015 Jan 09 & $K_{c}$ & 512 & 12.0 & $\cdots$ & 12 \\
\hline HD 114783 & 0 & 2014 Jun 09 & $K_{c}$ & 1024 & 13.6 & $\cdots$ & 12 \\
\hline HD 115617 & 0 & 2014 Jun 09 & $K_{c}$ & 256 & 13.5 & $\ldots$ & 13 \\
\hline
\end{tabular}


Table 1

(Continued)

\begin{tabular}{|c|c|c|c|c|c|c|c|}
\hline Target & $N_{c c}$ & UT Obs. Date & Filter & Array & $T_{\text {int }}(\mathrm{s})$ & $N_{\text {fit }}$ & $N_{\text {stack }}$ \\
\hline \multirow[t]{5}{*}{ HD 116029} & 1 & 2013 Jul 04 & $K_{c}$ & 512 & 10.0 & 11 & 12 \\
\hline & & 2014 Jun 09 & $K_{c}$ & 1024 & 13.6 & 12 & 12 \\
\hline & & 2014 Jun 09 & $J_{c}$ & 512 & 13.2 & 11 & $\cdots$ \\
\hline & & 2015 Jan 09 & $K_{c}$ & 512 & 12.0 & 12 & 12 \\
\hline & & 2015 Jan 09 & $J_{c}$ & 512 & 12.7 & 12 & $\cdots$ \\
\hline HD 117176 & 0 & 2013 Jul 04 & $K_{c}$ & 256 & 18.0 & $\ldots$ & 12 \\
\hline HD 117207 & 0 & 2014 Jun 09 & $K_{c}$ & 1024 & 12.5 & $\cdots$ & 14 \\
\hline \multirow[t]{2}{*}{ HD 125612} & 0 & 2014 Jun 09 & $K_{c}$ & 1024 & 15.0 & $\cdots$ & 12 \\
\hline & & 2015 Jun 24 & $K_{c}$ & 1024 & 15.0 & $\cdots$ & 12 \\
\hline \multirow[t]{4}{*}{ HD 126614} & 1 & 2014 Jun 09 & $K_{s}$ & 1024 & 13.6 & 12 & 12 \\
\hline & & 2015 Jan 09 & $K_{c}$ & 1024 & 12.5 & 12 & 12 \\
\hline & & 2015 Jun 24 & $K_{c}$ & 1024 & 12.5 & 12 & 12 \\
\hline & & 2015 Jun 24 & $J_{c}$ & 1024 & 12.5 & 12 & $\ldots$ \\
\hline HD 128311 & 0 & 2014 Jul 07 & $K_{c}$ & 512 & 10.6 & $\ldots$ & 12 \\
\hline HD 130322 & 0 & 2014 Jul 12 & $K_{c}$ & 1024 & 13.6 & $\ldots$ & 12 \\
\hline HD 131496 & 0 & 2013 Jul 04 & $K_{c}$ & 512 & 10.0 & $\ldots$ & 12 \\
\hline \multirow[t]{2}{*}{ HD 134987} & 0 & 2016 Jun 09 & $K_{c}$ & 1024 & 15.0 & $\cdots$ & 12 \\
\hline & & 2016 Jun 09 & $J_{c}$ & 1024 & 15.0 & $\ldots$ & $\ldots$ \\
\hline HD 141399 & 0 & 2014 May 21 & $K_{c}$ & 1024 & 13.6 & $\cdots$ & 12 \\
\hline HD 141937 & 0 & 2013 Jul 04 & $K_{c}$ & 1024 & 9.0 & $\cdots$ & 12 \\
\hline \multirow[t]{5}{*}{ HD 142245} & 2 & $2013 \mathrm{Jul} 04$ & $K_{c}$ & 512 & 10.6 & 15 & 15 \\
\hline & & 2014 Jun 09 & $K_{c}$ & 512 & 13.2 & 11 & 12 \\
\hline & & 2014 Jun 09 & $J_{c}$ & 512 & 13.2 & 10 & $\ldots$ \\
\hline & & 2015 Jun 24 & $K_{c}$ & 768 & 12.7 & 12 & 12 \\
\hline & & 2016 Jun 09 & $K_{c}$ & 512 & 15.9 & 11 & 12 \\
\hline HD 143761 & 0 & 2014 Jun 09 & $K_{c}$ & 256 & 13.5 & $\cdots$ & 12 \\
\hline HD 145675 & 0 & 2014 Jul 12 & $K_{c}$ & 512 & 15.9 & $\cdots$ & 12 \\
\hline HD 145934 & 0 & 2014 May 21 & $K_{c}$ & 1024 & 12.5 & $\cdots$ & 12 \\
\hline HD 149143 & 0 & 2013 Jul 04 & $K_{c}$ & 1024 & 9.0 & $\ldots$ & 12 \\
\hline HD 152581 & 0 & 2013 Jul 04 & $K_{c}$ & 1024 & 9.0 & $\cdots$ & 12 \\
\hline HD 154345 & 0 & 2014 Jul 12 & $K_{c}$ & 512 & 15.9 & $\cdots$ & 12 \\
\hline HD 156279 & 0 & 2013 Aug 19 & $K_{c}$ & 1024 & 9.0 & $\ldots$ & 12 \\
\hline HD 156668 & 0 & $2013 \mathrm{Jul} 04$ & $K_{c}$ & 1024 & 9.0 & $\cdots$ & 12 \\
\hline HD 158038 & 0 & 2015 Jun 24 & $K_{c}$ & 768 & 12.7 & $\ldots$ & 12 \\
\hline HD 163607 & 0 & 2013 Aug 19 & $K_{c}$ & 1024 & 9.0 & $\cdots$ & 12 \\
\hline \multirow[t]{5}{*}{ HD 164509} & 1 & 2014 Jun 09 & $J_{c}$ & 1024 & 12.5 & 11 & $\cdots$ \\
\hline & & 2014 Jun 09 & $K_{c}$ & 1024 & 12.5 & 12 & 12 \\
\hline & & 2015 Jun 24 & $K_{c}$ & 1024 & 12.5 & 12 & 12 \\
\hline & & 2016 Jun 09 & $J_{c}$ & 512 & 15.9 & 12 & $\ldots$ \\
\hline & & 2016 Jun 09 & $K_{c}$ & 1024 & 15.0 & 12 & 12 \\
\hline HD 164922 & 0 & 2015 Jun 24 & $K_{c}$ & 768 & 12.7 & $\ldots$ & 12 \\
\hline \multirow[t]{2}{*}{ HD 168443} & 0 & 2013 Aug 19 & $J_{c}$ & 512 & 10.6 & $\ldots$ & $\ldots$ \\
\hline & & 2013 Aug 19 & $K_{c}$ & 512 & 10.0 & $\ldots$ & 12 \\
\hline HD 168746 & 0 & 2013 Aug 19 & $K_{c}$ & 1024 & 9.0 & $\cdots$ & 12 \\
\hline HD 169830 & 0 & 2013 Aug 19 & $K_{c}$ & 512 & 10.6 & $\cdots$ & 12 \\
\hline HD 170469 & 0 & 2013 Jul 04 & $K_{c}$ & 1024 & 9.0 & $\cdots$ & 12 \\
\hline HD 175541 & 0 & 2013 Jul 04 & $K_{c}$ & 1024 & 9.0 & $\ldots$ & 12 \\
\hline \multirow[t]{5}{*}{ HD 177830} & 1 & 2014 Jun 09 & $J_{c}$ & 512 & 13.2 & 9 & $\ldots$ \\
\hline & & 2014 Jun 09 & $K_{c}$ & 512 & 13.2 & 12 & 12 \\
\hline & & 2015 Jul 05 & $K_{c}$ & 512 & 13.2 & 12 & 12 \\
\hline & & 2016 Jun 09 & $J_{c}$ & 512 & 15.9 & 12 & $\ldots$ \\
\hline & & 2016 Jun 09 & $K_{c}$ & 512 & 15.9 & 12 & 12 \\
\hline HD 178911B & 0 & 2013 Aug 19 & $K_{c}$ & 1024 & 9.0 & $\ldots$ & 12 \\
\hline HD 179079 & 0 & 2013 Aug 19 & $K_{c}$ & 1024 & 9.0 & $\ldots$ & 12 \\
\hline HD 179949 & 0 & 2013 Aug 19 & $K_{c}$ & 512 & 10.6 & $\ldots$ & 12 \\
\hline HD 180902 & 0 & 2014 Jul 12 & $K_{c}$ & 1024 & 13.6 & $\ldots$ & 12 \\
\hline HD 181342 & 0 & 2013 Aug 19 & $K_{c}$ & 512 & 10.6 & $\cdots$ & 12 \\
\hline HD 183263 & 0 & $2015 \mathrm{Jul} 05$ & $K_{c}$ & 1024 & 15.0 & $\ldots$ & $\ldots$ \\
\hline HD 186427B & 0 & 2013 Aug 19 & $K_{c}$ & 512 & 10.6 & $\cdots$ & 12 \\
\hline \multirow[t]{2}{*}{ HD 187123} & 1 & 2013 Aug 19 & $K_{c}$ & 1024 & 9.0 & 9 & 12 \\
\hline & & 2015 Jun 24 & $K_{c}$ & 1024 & 15.0 & 12 & 12 \\
\hline HD 188015 & 1 & 2013 Aug 19 & $J_{c}$ & 1024 & 9.0 & 6 & $\ldots$ \\
\hline
\end{tabular}


Table 1

(Continued)

\begin{tabular}{|c|c|c|c|c|c|c|c|}
\hline Target & $N_{c c}$ & UT Obs. Date & Filter & Array & $T_{\text {int }}(\mathrm{s})$ & $N_{\text {fit }}$ & $N_{\text {stack }}$ \\
\hline & & 2013 Aug 19 & $K_{c}$ & 1024 & 9.0 & 8 & 12 \\
\hline & & 2014 Jul 12 & $J_{c}$ & 1024 & 15.0 & 12 & $\ldots$ \\
\hline & & 2014 Jul 12 & $K_{c}$ & 1024 & 15.0 & 12 & 12 \\
\hline & & 2013 Aug 19 & $K_{c}$ & 1024 & 9.0 & $\cdots$ & 12 \\
\hline HD 190360 & 0 & 2014 Jul 12 & $K_{c}$ & 512 & 15.9 & $\cdots$ & 12 \\
\hline HD 192263 & 0 & 2013 Aug 19 & $K_{c}$ & 512 & 10.6 & $\cdots$ & 12 \\
\hline \multirow{5}{*}{ HD 195019} & & 2013 Aug 19 & $J_{c}$ & 512 & 10.6 & 6 & $\cdots$ \\
\hline & & 2014 Jun 09 & $K_{c}$ & 1024 & 13.6 & 12 & 12 \\
\hline & & 2014 Jul 12 & $K_{c}$ & 1024 & 13.6 & 12 & 12 \\
\hline & & 2014 Jul 12 & $J_{c}$ & 512 & 15.9 & 12 & $\cdots$ \\
\hline & & 2015 Jun 03 & $K_{c}$ & 768 & 12.7 & 12 & 12 \\
\hline & & $2014 \mathrm{Jul} 12$ & $K_{c}$ & 1024 & 15.0 & 12 & 12 \\
\hline & & 2014 Jul 12 & $J_{c}$ & 1024 & 15.0 & 9 & $\cdots$ \\
\hline & & $2015 \mathrm{Jul} 05$ & $K_{c}$ & 1024 & 20.0 & 12 & 12 \\
\hline & & $2015 \mathrm{Jul} 05$ & $J_{c}$ & 1024 & 20.0 & 12 & $\cdots$ \\
\hline & & 2015 Oct 26 & $K_{c}$ & 1024 & 15.0 & 12 & 12 \\
\hline & & 2016 Sep 13 & $K_{c}$ & 1024 & 12.5 & 3 & 3 \\
\hline HD 209458 & 0 & 2013 Jun 22 & $K_{s}$ & 256 & 9.0 & $\cdots$ & 12 \\
\hline HD 210277 & 0 & 2014 Nov 07 & $K_{c}$ & 1024 & 13.6 & $\cdots$ & 15 \\
\hline HD 212771 & 0 & 2013 Aug 19 & $K_{c}$ & 512 & 12.5 & $\cdots$ & 12 \\
\hline HD 217014 & 0 & 2013 Aug 19 & $K_{c}$ & 256 & 9.0 & $\cdots$ & 12 \\
\hline HD 217107 & 0 & 2013 Nov 17 & $K_{c}$ & 512 & 10.6 & $\cdots$ & 15 \\
\hline & & 2016 Sep 12 & $K_{s}$ & 1024 & 12.0 & 12 & 12 \\
\hline KELT-4A & 2 & 2015 Dec 20 & $K_{s}$ & 1024 & 15.0 & 12 & 12 \\
\hline \multirow[t]{3}{*}{ WASP-12 } & 2 & 2014 Dec 04 & $K_{s}$ & 1024 & 12.0 & 13 & 13 \\
\hline & & 2015 Dec 26 & $K_{s}$ & 1024 & 15.0 & 12 & 12 \\
\hline & & 2016 Sep 13 & $K_{s}$ & 1024 & 30.0 & 4 & 4 \\
\hline
\end{tabular}

Note. Column $N_{c c}$ is the number of candidate companions detected. Column "Array" is the horizontal size, in pixels, of the NIRC2 array readout region and corresponds to subarray sizes of $1024 \times 1024$ (the full NIRC2 array), $768 \times 760,512 \times 512$, or $256 \times 264$. Column $T_{\text {int }}$ is the total integration time, in seconds, of a single frame. Column $N_{\text {fit }}$ is the number of frames used in our photometric and/or astrometric analysis, and is only given when companions are present. Column $N_{\text {stack }}$ is the number of frames combined to make the contrast curve measurements. We only compute contrast curves in the $K_{s}$ and $K_{c}$ bandpasses so this column is not applicable for other bandpasses. In some cases, $N_{\text {fit }}$ and $N_{\text {stack }}$ are not equal because the companion may not be present in all frames due to the dither pattern and/or observing conditions.

product of different formation pathways. Therefore, it is important to study formation and migration processes on a wide range of planetary separations.

Eggenberger et al. (2007) carried out the most comprehensive survey thus far, searching around 56 known RV-planet host stars as well as a control group of 74 stars without a planetary signal. Both their planet-hosting and control samples were from a CORALIE RV planet survey (Udry et al. 2000). Considering only companion candidates that they have assessed as likely or truly bound, the planet sample had a companion rate of $6 / 56$, while the control group had a larger companion rate of $13 / 74$. Since this study, there have only been a few other surveys (Ginski et al. 2012; Mugrauer \& Ginski 2015; Ginski et al. 2016) searching for companion stars to RV-detected planet hosts, all with similar sample sizes and target lists. In total, these surveys found 17 systems with RV-detected giant planets and stellar companions within $6 "$. 
In this work, we used the Keck Observatory to conduct the largest stellar companion search around RV-detected giant planet host stars to date. These stars host giant planets with orbital semimajor axes ranging from 0.01 to $5 \mathrm{au}$, including hot Jupiters, warm Jupiters, and cool Jupiters. Because Eggenberger et al. (2007) and Mugrauer \& Ginski (2015) conducted their diffraction-limited AO surveys with the VLT in the southern hemisphere, our sample of 144 targets contains 119 unique new targets without previous diffraction-limited imaging from observatories similar in size to Keck. Ginski et al. (2012, 2016) carried out a "lucky imaging" survey with the $2.2 \mathrm{~m}$ Calar Alto observatory in the northern hemisphere. Although lucky imaging surveys are less sensitive to close stellar companions, our sample contains 72 unique targets not present in either the VLT or Calar Alto surveys. In Bryan et al. (2016), we searched for long-term RV trends around the same stars to find planetary companions; however, we excluded 23 stars with fewer than 12 Keck RV measurements in order to ensure good constraints on detected RV trends. For the three triple star systems in our sample, we combine our new astrometric measurements with previous measurements in order to fit the orbits of the binary star companions around their center of mass. We also include additional observations of three triple systems with transiting planets that were detected in previous surveys. Unlike the relatively wide separation binaries in our sample, the secondary and tertiary companions in these hierarchical triple systems have a much shorter mutual orbital period, allowing us to detect orbital motion with a several year baseline. For transiting planet systems, we show that imaging of these triple systems can constrain the inclination of the stellar orbits relative to that of the planetary orbit.

In Section 2, we describe our observational campaign. In Section 3, we describe our photometric and astrometric analysis of candidate stellar companions and provide detection limits for all observed stars. In Section 4, we discuss each detected multistellar system individually. In Section 5, we compare our results to other surveys, discuss the implications on giant planet formation, and characterize the orbits of companion stars in our hierarchical triple systems. Finally, we present a summary in Section 6.

\section{Observations}

We obtained infrared AO images of 144 stars with RV-detected giant planets in order to search for stellar companions. This sample includes the set of AO images used to constrain the masses and orbits of the RVdetected companions described in Bryan et al. (2016), except for two systems. We exclude HD 33636 and HD 190228 because subsequent studies revealed that their companions are actually stars on very close orbits. The companion to HD 33636 is an M-dwarf star on a 2117 day orbit (Bean et al. 2007) and the companion to HD 190228 is a brown dwarf on a 1146 day orbit (Sahlmann et al. 2011). All target stars are part of the California Planet Survey (Howard et al. 2010). We conducted our survey with the NIRC2 instrument (instrument PI: Keith Matthews) on Keck II using Natural Guide Star AO (Wizinowich 2013) from 2013 August to 2016 September. The observations are listed in Table 1 . We follow the procedure in Ngo et al. (2015), which we briefly describe here. We operated NIRC2 in natural guide star mode and used the narrow camera setting, which has a pixel scale of 10 mas pixel ${ }^{-1}$. The majority of our targets were bright enough ( $\mathrm{K}$ magnitudes from 1.8 to 8.1 ) to saturate the NIRC2 detector in $K_{s}$ band, so we used the narrower $K_{c}$ bandpass $(2.2558-2.2854 \mu \mathrm{m})$ instead to search for companions. For systems where we detected a candidate companion, we also obtained $J_{c}$ images to measure a $J_{c}-K_{c}$ color. We determine whether each candidate companion is physically bound using a second epoch of $K_{c}$ images taken one to three years later, which allows us to check for common proper motion. We flat-field and dark-subtract our data and apply a spatial filer to remove bad pixels, as described in Ngo et al. (2015). We made photometric and astrometric measurements using individually calibrated frames and computed contrast curves using a median stack of these individually calibrated frames.

In addition to the $144 \mathrm{RV}$-detected planet host stars in our main survey sample, we also obtained images of three additional transiting planet host stars previously known to be in triple systems. Two of these triple systems (HAT-P-8, WASP-12) were previously discovered by imaging surveys (Bergfors et al. 2013; Ginski et al. 2013) and later characterized as part of our "Friends of hot Jupiters" program (Bechter et al. 2014; Ngo et al. 2015). The other triple system (KELT4A, also known as HIP-51260) was recently reported by Eastman et al. (2016). Although we do not include these additional triple systems when determining the overall multiplicity rate for planet-hosting stars, we obtain and process the images of these additional systems in the same way as our survey targets. Table 2 lists the properties of the stars from our survey with detected companions as well as the separate sample of previously published triple systems.

\section{Analysis}

\subsection{Photometry and Astrometry of Candidate Multi-stellar Systems}

We detect candidate companions around 13 stars in our survey (see Figures 1 and 2). We summarize the stellar parameters for stars with detected companions as well as our determination of the companion's bound or background status in Table 2. As described in Ngo et al. (2015), we model the stellar point-spread function (PSF) as a combination of a Moffat and Gaussian function. We use a maximum likelihood estimation routine to find the best-fit parameters of a multiplesource PSF for each candidate multi-stellar system and determine the flux ratio of the candidate companion to the primary star, as well as the on-sky separation. On 2015 April 13, the optics in the Keck II AO bench were realigned to improve performance. We account for the NIRC2 detector distortion and rotation using astrometric solutions from Yelda et al. (2010) for data taken prior to this realignment work and from Service et al. (2016) for data taken afterward. To determine the stability of the Yelda et al. (2010) solution (based on data from 2007 to 2009), Service et al. (2016) also computed a distortion solution for data taken just prior to the NIRC2 realignment. The Service et al. (2016) and Yelda et al. (2010) solutions are consistent within 0.5 milliarcseconds, demonstrating that the Yelda et al. (2010) solution is suitable for all of our NIRC2 data taken prior to 2015 April 13 UT. Our reported uncertainties include both measurement errors and the uncertainty contributed by the published astrometric solution. We report the fluxes and astrometry for each candidate companion in Tables 3 and 4. 
Table 2

Stellar Parameters for Candidate Multi-stellar Systems

\begin{tabular}{|c|c|c|c|c|c|c|c|c|}
\hline \multirow{2}{*}{ Target } & \multirow{2}{*}{ Type } & \multirow{2}{*}{$\begin{array}{l}T_{\text {eff }} \\
(\mathrm{K})\end{array}$} & \multirow{2}{*}{$\begin{array}{c}M \\
\left(M_{\odot}\right)\end{array}$} & \multirow{2}{*}{$\begin{array}{l}\log g \\
(\mathrm{cgs})\end{array}$} & \multirow{2}{*}{$\begin{array}{c}D \\
\text { (pc) }\end{array}$} & \multicolumn{2}{|c|}{ References for... } & \\
\hline & & & & & & $T$ & $M, \log g$ & $D$ \\
\hline HD 30856 & $\mathrm{~B}$ & $4982 \pm 44$ & $1.350 \pm 0.094$ & $3.40 \pm 0.06$ & $118_{-9}^{+11}$ & 1 & 1 & \\
\hline HD 43691 & $\mathrm{~T}$ & $6200 \pm 40$ & $1.38 \pm 0.05$ & $4.28 \pm 0.13$ & $81_{-5}^{+6}$ & 3 & 3 & \\
\hline HD 86081 & B & $6036 \pm 23$ & $1.23 \pm 0.08$ & $4.21 \pm 0.04$ & $95_{-8}^{+10}$ & 4 & 4 & \\
\hline HD 126614 & B & $5585 \pm 44$ & $1.145 \pm 0.030$ & $4.39 \pm 0.08$ & $73 \pm 5$ & 6 & 6 & \\
\hline HD 142245 & $\mathrm{~T}$ & $4878 \pm 44$ & $1.69 \pm 0.12$ & $3.30 \pm 0.06$ & $110_{-7}^{+8}$ & 1 & 1 & \\
\hline HD 164509 & B & $5922 \pm 44$ & $1.13 \pm 0.02$ & $4.44 \pm 0.06$ & $52 \pm 3$ & 7 & 7 & \\
\hline HD 177830 & $\mathrm{~B}$ & $5058 \pm 35$ & $1.37 \pm 0.04$ & $3.66 \pm 0.06$ & $59 \pm 2$ & 8 & 8 & \\
\hline HD 187123 & bg & $5845 \pm 22$ & $1.037 \pm 0.025$ & $4.32 \pm 0.04$ & $48 \pm 1$ & 9 & 10 & \\
\hline HD 188015 & bg & $5746^{\mathrm{a}}$ & $1.056 \pm 0.09$ & $4.41_{-0.04}^{+0.05}$ & $57 \pm 3$ & 11 & 7 & \\
\hline KELT-4A & $\mathrm{T}$ & $6207 \pm 75$ & $1.204 \pm 0.070$ & $4.105_{-0.032}^{+0.029}$ & $211_{-12}^{+13}$ & 16 & 16 & 16 \\
\hline WASP-12 & $\mathrm{T}$ & $6118 \pm 64$ & $1.38 \pm 0.19$ & $4.159 \pm 0.024$ & $427 \pm 90$ & 13 & 17 & 15 \\
\hline
\end{tabular}

Note. The "Type" column indicates whether the candidate multi-stellar system is a bound binary (B), bound triple (T), or a background object (bg). The planetary parameters are listed only for the innermost planet in each system in our RV planet host star survey. All planetary parameters are listed as they appear in the cited reference.

${ }^{a}$ No uncertainty is available for this temperature estimate. Since the candidate companion is a background object, the temperature was not used in any further calculation.

References. (1) Johnson et al. (2011), (2) van Leeuwen (2007), (3) Da Silva et al. (2007), (4) Santos et al. (2013), (5) Jofré et al. (2015), (6) Howard et al. (2010), (7) Giguere et al. (2012), (8) Johnson et al. (2006), (9) Santos et al. (2004), (10) Takeda et al. (2007), (11) Butler et al. (2006), (12) Ghezzi et al. (2010), (13) Torres et al. (2012), (14) Mancini et al. (2013), (15) Triaud et al. (2014), (16) Eastman et al. (2016), (17) Southworth (2012).

\subsection{Common Proper Motion Check}

We obtained a second epoch of $K_{c}$ images of all candidate companions to determine whether these companions are gravitationally bound to the primary star. As described in Ngo et al. (2015), we show the measured projected separation and position angle of each candidate companion as a function of time and compare it to the predicted tracks for a bound companion and an infinitely distant background object in Figures 3 and 4. Predicted tracks are computed using stellar proper motions from van Leeuwen (2007) and start from the epoch with the smallest uncertainties. When available, we also include previously published astrometric measurements and their corresponding uncertainties in Figures 3 and 4 and Table 4. After reviewing the available astrometry, we conclude that 11 out of 13 candidate multi-stellar systems are gravitationally bound. We discuss the astrometric measurements for each individual system separately in Section 4.

\subsection{Companion Star Masses and Separations}

For the 11 confirmed multi-stellar systems, we follow the procedure described in Ngo et al. (2015) to compute the companion star's physical parameters. Here, we will describe our method briefly. We model the stars with PHOENIX synthetic spectra (Husser et al. 2013) assuming solar compositions for both stars $([\mathrm{Fe} / \mathrm{H}]=0,[\alpha / \mathrm{H}]=0)$ and calculate fluxes from each star by integrating the chosen spectra over the observed bandpass. For the primary star, we interpolate PHOENIX spectra to get a model with the corresponding stellar mass, radius, and effective temperature as reported in previous studies, summarized in Table 2. Using the published parallax and corresponding distance to each system and the flux of the primary star, we solve for the companion temperature that best fits our measured photometric flux ratio. With this best-fitting effective temperature, we calculate the corresponding companion mass and radius using zero-age mainsequence star models (Baraffe et al. 1998). We report the properties of each companion star in Table 5 with uncertainties calculated from the uncertainty in the measured flux ratio. These errors do not account for systematic uncertainties from our use of PHOENIX spectra or the zero-age main-sequence model. We find that errors introduced by assuming solar metallicities and compositions are much smaller than the uncertainties on the measured contrast ratio. Similarly, some error may be introduced from using literature values for primary star mass and radius because these measurements may not have included the effects of the secondary star. Because the secondary stars are several magnitudes or more fainter, this effect is also smaller than the uncertainties. For planet population and orbit fit analyses presented in Section 5, we use the epoch with the smallest measurement error as the final measurement for each system.

\subsection{Contrast Curves for All Systems}

We report the $5 \sigma K_{c}$-band detection limits for each star in our survey as a function of the projected separation. For systems with a companion, we mask out the companion before calculating this detection limit. Figure 5 shows the contrast curves for the stars in this work. We compute the contrast 

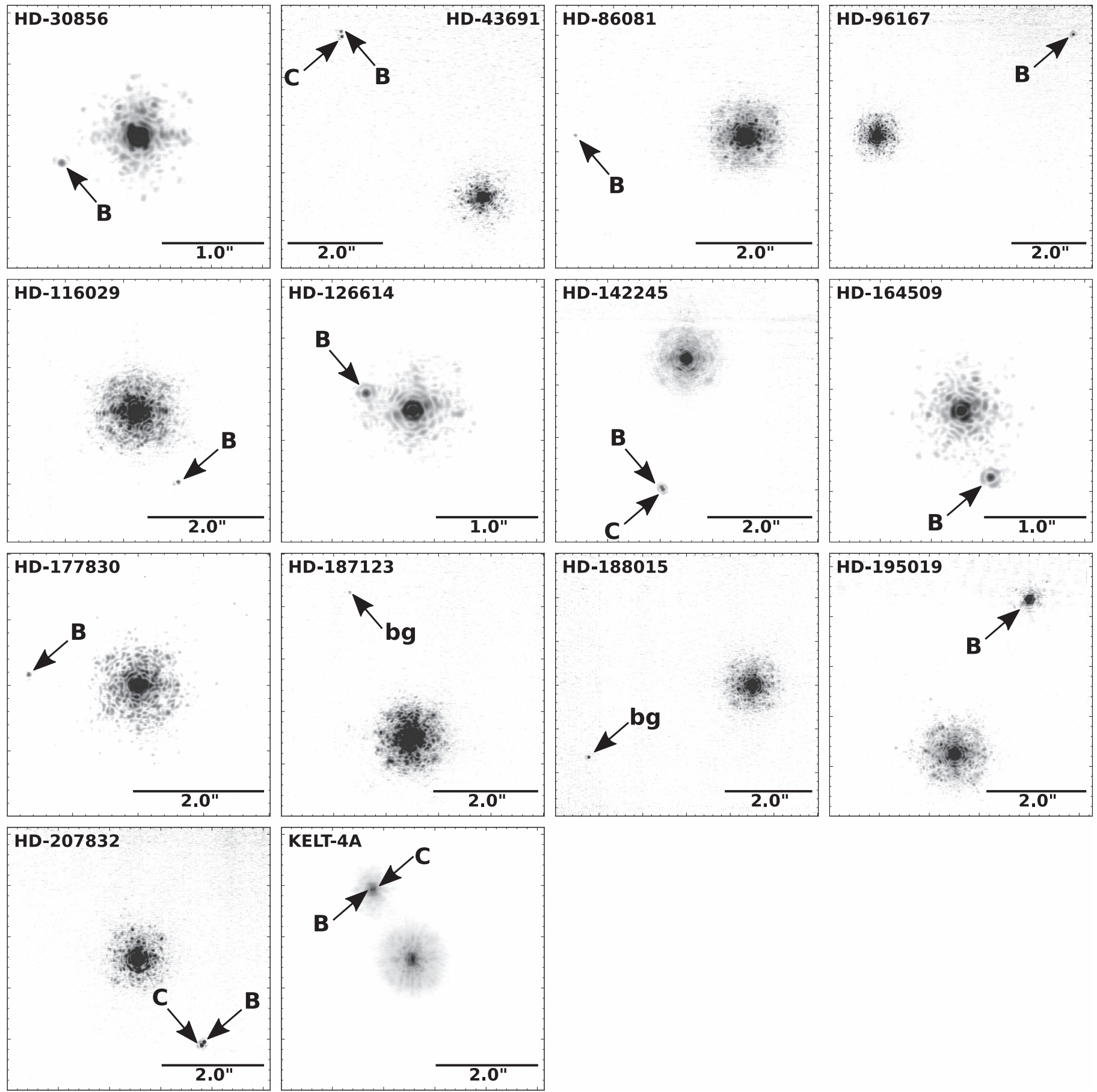

Figure 1. Median-stacked K-band image for each candidate mutli-stellar system. Each image is oriented such that north points up and east is to the left. Confirmed comoving companions are indicated by capital letters while candidates determined to be background objects are labelled as "bg." The KELT-4A triple system was not part of our main survey (see Section 2).

curves from the standard deviation of pixel values in a series of annuli, following the procedure described in Ngo et al. (2015), and provide a complete list of these curves for each individual system in Table 10. For targets imaged on the full $1024 \times 1024$ array, we do not have coverage in all directions beyond $5^{\prime \prime}$ and drop to $90 \%$ directional completeness at $6 "$. This limit is smaller for targets imaged on smaller subarrays. This lack of directional completeness results in fewer frames imaged in that region, which increases the standard deviation of stacked pixels and leads to lower contrast.

\section{Individual Systems}

Tables 2-5 summarize our survey targets' properties, measured companion photometry, measured companion astrometry, and calculated companion properties, respectively. Table 4 also includes astrometric measurements from other studies, when available. The following paragraphs provide additional notes on each of the 11 confirmed multi-stellar systems from our survey as well as candidate companions that were found to be background objects. In total, we report the discovery of three new multi-stellar planet-hosting systems 

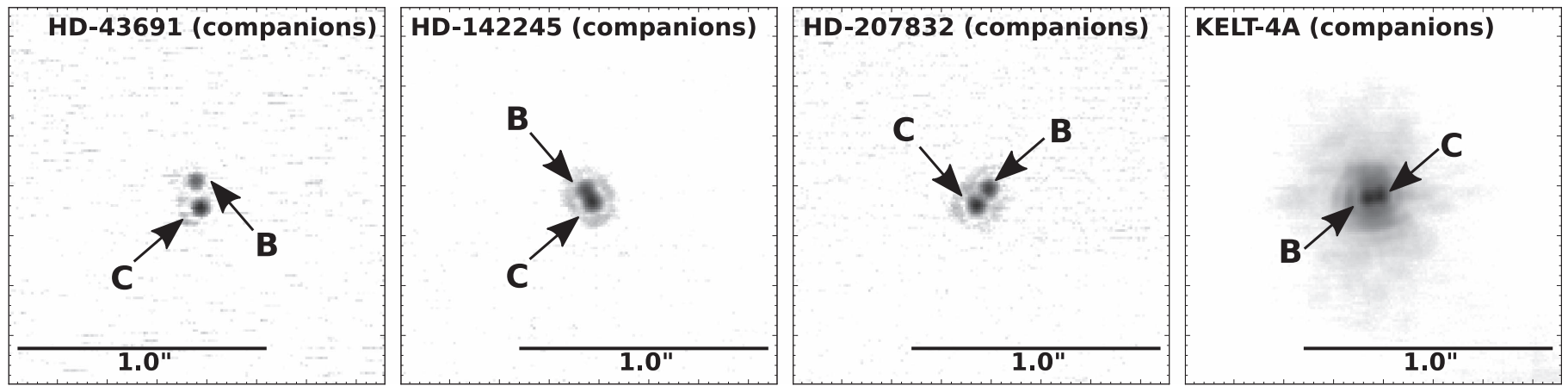

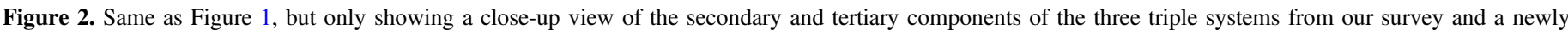
reported triple system, KELT-4A.

(HD 30856, HD 86081, and HD 207832) and the first confirmation of common proper motion for three additional systems (HD 43691, HD 116029, and HD 164509).

HD 30856. This binary system is reported for the first time in this work. Our images from 2014 and 2015 confirm that this companion is comoving with its host star.

HD 43691. Our images from 2013 through 2016 provide the first confirmation that this is a comoving hierarchical triple system. The secondary and tertiary components have a projected separation of 360 au from the primary star, and a mutual projected separation of $10 \mathrm{au}$. Ginski et al. (2016) reported a single companion to this system that is consistent with our detection. They did not resolve the individual secondary and tertiary components but they did note that the companion appeared to have an elongated PSF. We are unable to use their astrometric measurements in our analysis because they did not resolve the two companion stars. We label the primary star as "A," the northernmost companion as "B" and the other companion as "C."

$H D$ 86081. This binary system is presented for the first time in this work. In Bryan et al. (2016), we report a long-term RV trend of $-1.3 \pm 0.25 \mathrm{~m} \mathrm{~s}^{-1} \mathrm{yr}^{-1}$ in this system corresponding to a companion with a minimum mass of 0.69 Jupiter masses at a separation of $4.6 \mathrm{au}$. Here, we report a companion with a mass of $88 \pm 2$ Jupiter masses $\left(0.0840 \pm 0.002 M_{\odot}\right)$ and a projected separation of $280 \pm 30$ au. To determine whether or not our imaged companion could be responsible for the measured RV trend, we calculate the minimum companion star mass required to produce the observed RV trend at this projected separation using Equation (6) from Torres (1999). This minimum mass is $1.4_{-0.5}^{+0.6} M_{\odot}$, indicating that the companion star is not responsible for the RV trend. The nondetection of an additional companion in the AO images set an upper limit on the RV trend companion to be 72 Jupiter masses at 124 au. Our images from 2013 and 2014 indicate that the stellar companion is comoving with its host star.

$H D$ 96167. This binary system was previously reported by Mugrauer \& Ginski (2015) to be a comoving companion to its host star. Their astrometric measurements date back to 2013 and are consistent with our measurements in 2014 and 2015.

HD 116029. Ginski et al. (2016) reported this as a candidate binary system based on their 2013 image but were unable to confirm that the companion was comoving because they only had one epoch of astrometry. Our measured separation and position angle from our 2013, 2014, and 2015 images are consistent with their measurement. We provide the first confirmation that this system is a comoving binary pair.
HD 126614. The close companion star in this system was first detected in 2009 by Howard et al. (2010). Ginski et al. (2012) also imaged this system in 2011 and concluded that the companion is comoving. Our images from 2014 and 2015 agree with this assessment. We also found a long-term RV trend for this system in Bryan et al. (2016) that is consistent with the imaged stellar companion. Finally, this system also has an additional distant common proper motion companion, NLTT 37349, at 41!"8 (3000 au; Lodieu et al. 2014) that is outside of our survey's field of view.

HD 142245. Our images from 2013 through 2016 provide the first images that resolve the individual stars in this hierarchical triple system. The two companion stars have a projected separation of $280 \mathrm{au}$ from the primary star and a mutual projected separation of 6 au. Mugrauer \& Ginski (2015) reported images from 2012 and 2013 showing a companion with an elongated PSF that is consistent with our measurements. They determined that this companion was comoving with the host star but were unable to resolve the individual components. We label the primary star as "A," the northernmost companion as "B" and the other companion as " $\mathrm{C}$."

$H D$ 164509. Our images from 2014, 2015, and 2016 provide the first confirmation that this is a comoving binary system. Wittrock et al. (2016) also report a companion from their 2014 image that is consistent with our detection. With only one epoch, they were unable to confirm whether the companion is bound, but they noted that the color of the companion was consistent with a lower mass star at the same distance as the target star. We also found a long-term RV trend for this system in Bryan et al. (2016) that is consistent with the imaged stellar companion.

$H D$ 177830. The companion to this star was first reported by Eggenberger et al. (2007). They used images from 2004 ( $H$-band) and 2005 ( $K$-band) to determine that this is a comoving binary system. Roberts et al. $(2011,2015)$ later combined their images of this binary system with additional observations dating back to 2002. Our images in 2014, 2015, and 2016 recover the same companion reported in these previous studies and support the conclusion that the companion is bound.

HD 195019. Fischer et al. (1999) reported a companion around this star, but did not have precise measurements of its photometry or astrometry. Eggenberger et al. (2004) subsequently noted that both components are comoving based on archival data from Fischer et al. (1999), Allen et al. (2000), and Patience et al. (2002). Roberts et al. (2011) published additional images from 2002, but did not report uncertainties on their astrometry. Our images from 2013 through 2015 are 
Table 3

Photometry of Confirmed Stellar Companions

\begin{tabular}{|c|c|c|c|c|c|c|}
\hline Companion & UT Obs. Date & $m_{J c}$ & $m_{K c}$ & $\Delta J_{c}$ & $\Delta K_{c}$ & $J_{c}-K_{c}$ \\
\hline \multicolumn{7}{|c|}{$R V$ planet host stars } \\
\hline \multirow[t]{3}{*}{ HD $30856 B$} & 2014 Oct 04 & $10.963 \pm 0.020$ & $10.904 \pm 0.021$ & $4.708 \pm 0.020$ & $5.247 \pm 0.021$ & $0.059 \pm 0.029$ \\
\hline & 2014 Dec 07 & $11.160 \pm 0.088$ & $10.473 \pm 0.042$ & $4.905 \pm 0.088$ & $4.816 \pm 0.042$ & $0.687 \pm 0.097$ \\
\hline & 2015 Oct 26 & $\ldots$ & $10.786 \pm 0.046$ & $\ldots$ & $5.129 \pm 0.046$ & $\ldots$ \\
\hline \multirow[t]{4}{*}{ HD 43691B } & 2013 Dec 18 & $\ldots$ & $13.698 \pm 0.084$ & $\ldots$ & $6.998 \pm 0.084$ & $\ldots$ \\
\hline & 2014 Dec 04 & $14.38 \pm 0.38$ & $13.253 \pm 0.051$ & $7.40 \pm 0.38$ & $6.553 \pm 0.051$ & $1.12 \pm 0.38$ \\
\hline & 2015 Oct 26 & $\ldots$ & $13.203 \pm 0.093$ & $\ldots$ & $6.503 \pm 0.093$ & $\ldots$ \\
\hline & 2016 Sep 13 & $\cdots$ & $12.94 \pm 0.11$ & $\ldots$ & $6.24 \pm 0.11$ & $\cdots$ \\
\hline \multirow[t]{4}{*}{ HD $43691 C$} & 2013 Dec 18 & $\ldots$ & $12.895 \pm 0.081$ & $\ldots$ & $6.195 \pm 0.081$ & $\ldots$ \\
\hline & 2014 Dec 04 & $23.0 \pm 3.0$ & $12.534 \pm 0.050$ & $16.0 \pm 3.0$ & $5.834 \pm 0.050$ & $10.5 \pm 3.0$ \\
\hline & 2015 Oct 26 & $\ldots$ & $12.483 \pm 0.044$ & $\ldots$ & $5.783 \pm 0.044$ & $\ldots$ \\
\hline & 2016 Sep 13 & $\cdots$ & $11.847 \pm 0.084$ & $\ldots$ & $5.147 \pm 0.084$ & $\ldots$ \\
\hline \multirow[t]{2}{*}{ HD 86081B } & 2013 Dec 18 & $\cdots$ & $15.25 \pm 0.35$ & $\cdots$ & $7.95 \pm 0.35$ & $\cdots$ \\
\hline & 2014 Dec 05 & $\cdots$ & $14.766 \pm 0.086$ & $\cdots$ & $7.467 \pm 0.086$ & $\cdots$ \\
\hline \multirow[t]{2}{*}{ HD 96167B } & 2014 Jan 12 & $\ldots$ & $12.97 \pm 0.40$ & $\cdots$ & $6.41 \pm 0.40$ & $\cdots$ \\
\hline & 2015 Jan 09 & $15.10 \pm 0.17$ & $12.591 \pm 0.039$ & $8.18 \pm 0.17$ & $6.037 \pm 0.039$ & $2.51 \pm 0.17$ \\
\hline \multirow[t]{3}{*}{ HD 116029B } & $2013 \mathrm{Jul} 04$ & $\ldots$ & $12.513 \pm 0.091$ & $\ldots$ & $6.979 \pm 0.091$ & $\ldots$ \\
\hline & 2014 Jun 09 & $13.63 \pm 0.16$ & $12.414 \pm 0.066$ & $7.49 \pm 0.16$ & $6.880 \pm 0.066$ & $1.22 \pm 0.17$ \\
\hline & 2015 Jan 09 & $13.85 \pm 0.11$ & $12.55 \pm 0.10$ & $7.71 \pm 0.11$ & $7.01 \pm 0.10$ & $1.30 \pm 0.15$ \\
\hline \multirow[t]{2}{*}{ HD 126614B } & 2015 Jan 09 & $\ldots$ & $11.132 \pm 0.018$ & $\ldots$ & $4.072 \pm 0.018$ & $\ldots$ \\
\hline & 2015 Jun 24 & $11.65 \pm 0.12$ & $10.9740 \pm 0.0100$ & $4.18 \pm 0.12$ & $3.9140 \pm 0.0100$ & $0.68 \pm 0.12$ \\
\hline \multirow[t]{4}{*}{ HD 142245B } & $2013 \mathrm{Jul} 04$ & $\ldots$ & $11.159 \pm 0.034$ & $\ldots$ & $6.049 \pm 0.034$ & $\ldots$ \\
\hline & 2014 Jun 09 & $11.241 \pm 0.080$ & $10.632 \pm 0.085$ & $5.552 \pm 0.080$ & $5.522 \pm 0.085$ & $0.61 \pm 0.12$ \\
\hline & 2015 Jun 24 & $\ldots$ & $10.896 \pm 0.037$ & $\ldots$ & $5.786 \pm 0.037$ & $\cdots$ \\
\hline & 2016 Jun 09 & $\cdots$ & $10.988 \pm 0.031$ & $\cdots$ & $5.878 \pm 0.031$ & $\cdots$ \\
\hline \multirow[t]{4}{*}{ HD $142245 \mathrm{C}$} & 2013 Jul 04 & $\ldots$ & $10.794 \pm 0.022$ & $\ldots$ & $5.684 \pm 0.022$ & $\ldots$ \\
\hline & 2014 Jun 09 & $11.164 \pm 0.060$ & $10.635 \pm 0.024$ & $5.475 \pm 0.060$ & $5.525 \pm 0.024$ & $0.529 \pm 0.064$ \\
\hline & 2015 Jun 24 & $\ldots$ & $10.690 \pm 0.013$ & $\ldots$ & $5.580 \pm 0.013$ & $\ldots$ \\
\hline & 2016 Jun 09 & $\ldots$ & $10.645 \pm 0.025$ & $\ldots$ & $5.535 \pm 0.025$ & $\ldots$ \\
\hline \multirow[t]{3}{*}{ HD 164509B } & 2014 Jun 09 & $11.66 \pm 0.27$ & $10.172 \pm 0.024$ & $4.72 \pm 0.27$ & $3.586 \pm 0.024$ & $1.49 \pm 0.27$ \\
\hline & 2015 Jun 24 & $\ldots$ & $10.022 \pm 0.053$ & $\ldots$ & $3.436 \pm 0.053$ & $\ldots$ \\
\hline & 2016 Jun 09 & $10.654 \pm 0.025$ & $10.0151 \pm 0.0056$ & $3.716 \pm 0.025$ & $3.4291 \pm 0.0056$ & $0.639 \pm 0.026$ \\
\hline \multirow[t]{3}{*}{ HD 177830B } & 2014 Jun 09 & $12.81 \pm 0.19$ & $11.861 \pm 0.081$ & $7.45 \pm 0.19$ & $7.052 \pm 0.081$ & $0.95 \pm 0.21$ \\
\hline & 2015 Jul 05 & $\ldots$ & $11.897 \pm 0.089$ & $\ldots$ & $7.088 \pm 0.089$ & $\cdots$ \\
\hline & 2016 Jun 09 & $12.339 \pm 0.045$ & $11.539 \pm 0.061$ & $6.972 \pm 0.045$ & $6.730 \pm 0.061$ & $0.800 \pm 0.076$ \\
\hline HD 195019B & 2013 Aug 19 & $8.511 \pm 0.018$ & $7.9276 \pm 0.0084$ & $2.911 \pm 0.018$ & $2.6676 \pm 0.0084$ & $0.583 \pm 0.020$ \\
\hline & 2014 Jun 09 & $\ldots$ & $7.970 \pm 0.059$ & $\ldots$ & $2.710 \pm 0.059$ & $\ldots$ \\
\hline & 2014 Jul 12 & $8.492 \pm 0.014$ & $7.9395 \pm 0.0069$ & $2.892 \pm 0.014$ & $2.6795 \pm 0.0069$ & $0.553 \pm 0.016$ \\
\hline & 2015 Jun 03 & $\ldots$ & $7.878 \pm 0.040$ & $\ldots$ & $2.618 \pm 0.040$ & $\ldots$ \\
\hline HD 207832B & 2013 Aug 19 & $14.58 \pm 0.18$ & $13.148 \pm 0.055$ & $7.00 \pm 0.18$ & $5.941 \pm 0.055$ & $1.44 \pm 0.19$ \\
\hline & 2014 Jul 12 & $14.336 \pm 0.094$ & $13.143 \pm 0.042$ & $6.749 \pm 0.094$ & $5.936 \pm 0.042$ & $1.19 \pm 0.10$ \\
\hline & $2015 \mathrm{Jul} 05$ & $\ldots$ & $12.870 \pm 0.086$ & $\ldots$ & $5.663 \pm 0.086$ & $\ldots$ \\
\hline & 2015 Oct 26 & $\cdots$ & $13.005 \pm 0.077$ & $\cdots$ & $5.798 \pm 0.077$ & $\ldots$ \\
\hline & 2016 Sep 13 & $\ldots$ & $13.47 \pm 0.12$ & $\ldots$ & $6.26 \pm 0.12$ & $\ldots$ \\
\hline & 2013 Aug 19 & $14.58 \pm 0.18$ & $13.166 \pm 0.033$ & $7.00 \pm 0.18$ & $5.959 \pm 0.033$ & $1.42 \pm 0.19$ \\
\hline HD $207832 \mathrm{C}$ & 2013 Aug 19 & $14.077 \pm 0.096$ & $12.813 \pm 0.048$ & $6.490 \pm 0.096$ & $5.606 \pm 0.048$ & $1.26 \pm 0.11$ \\
\hline & 2014 Jul 12 & $14.06 \pm 0.14$ & $12.826 \pm 0.070$ & $6.47 \pm 0.14$ & $5.619 \pm 0.070$ & $1.23 \pm 0.15$ \\
\hline & $2015 \mathrm{Jul} 05$ & $\ldots$ & $12.909 \pm 0.056$ & $\ldots$ & $5.702 \pm 0.056$ & $\ldots$ \\
\hline & 2015 Oct 26 & $\cdots$ & $12.921 \pm 0.044$ & $\ldots$ & $5.714 \pm 0.044$ & $\ldots$ \\
\hline & 2016 Sep 13 & $\ldots$ & $12.62 \pm 0.13$ & $\ldots$ & $5.41 \pm 0.13$ & $\ldots$ \\
\hline & 2013 Aug 19 & $14.077 \pm 0.096$ & $12.770 \pm 0.054$ & $6.490 \pm 0.096$ & $5.563 \pm 0.054$ & $1.31 \pm 0.11$ \\
\hline Transiting pla & ars in triple syst & & & & & \\
\hline HAТ-P-8B & 2014 Oct 03 & $\cdots$ & $14.883 \pm 0.082$ & $\ldots$ & $5.930 \pm 0.082$ & $\ldots$ \\
\hline & $2015 \mathrm{Jul} 07$ & $\cdots$ & $14.811 \pm 0.065$ & $\ldots$ & $5.858 \pm 0.065$ & $\ldots$ \\
\hline & 2016 Sep 12 & $\cdots$ & $14.803 \pm 0.058$ & $\ldots$ & $5.850 \pm 0.058$ & $\cdots$ \\
\hline HAT-P-8C & 2014 Oct 03 & $\ldots$ & $15.204 \pm 0.089$ & $\ldots$ & $6.251 \pm 0.089$ & $\ldots$ \\
\hline & $2015 \mathrm{Jul} 07$ & $\cdots$ & $15.469 \pm 0.025$ & $\ldots$ & $6.516 \pm 0.025$ & $\ldots$ \\
\hline & 2016 Sep 12 & $\cdots$ & $15.462 \pm 0.021$ & $\ldots$ & $6.509 \pm 0.021$ & $\ldots$ \\
\hline KELT-4A B & 2015 Dec 20 & $\cdots$ & $10.70 \pm 0.36$ & $\cdots$ & $2.02 \pm 0.36$ & $\cdots$ \\
\hline KELT-4A C & 2015 Dec 20 & $\cdots$ & $11.79 \pm 0.15$ & $\ldots$ & $3.10 \pm 0.15$ & $\ldots$ \\
\hline WASP-12B & 2014 Dec 04 & $\cdots$ & $13.251 \pm 0.077$ & $\cdots$ & $3.063 \pm 0.077$ & $\cdots$ \\
\hline & 2015 Dec 26 & $\cdots$ & $13.342 \pm 0.026$ & $\ldots$ & $3.154 \pm 0.026$ & $\ldots$ \\
\hline & 2016 Sep 13 & $\ldots$ & $13.294 \pm 0.035$ & $\ldots$ & $3.106 \pm 0.035$ & $\ldots$ \\
\hline WASP-12C & 2014 Dec 04 & $\cdots$ & $13.70 \pm 0.10$ & $\ldots$ & $3.51 \pm 0.10$ & $\cdots$ \\
\hline & 2015 Dec 26 & $\cdots$ & $13.411 \pm 0.023$ & $\ldots$ & $3.223 \pm 0.023$ & $\ldots$ \\
\hline & 2016 Sep 13 & $\cdots$ & $13.300 \pm 0.041$ & $\ldots$ & $3.112 \pm 0.041$ & $\ldots$ \\
\hline
\end{tabular}

(This table is available in machine-readable form.) 
Table 4

Astrometric Measurements of All Candidate Companions

\begin{tabular}{|c|c|c|c|c|c|}
\hline Candidate $^{\mathrm{a}}$ & UT Obs. Date & Band & $\rho$ (mas) & $\mathrm{PA}\left({ }^{\circ}\right)$ & References \\
\hline \multicolumn{6}{|c|}{$R V$ planet host stars } \\
\hline \multirow[t]{3}{*}{ HD 30856 B } & 2014 Oct 04 & $K_{c}$ & $789.4 \pm 1.5$ & $108.6 \pm 0.1$ & this work \\
\hline & 2014 Dec 07 & $K_{c}$ & $788.7 \pm 1.5$ & $108.7 \pm 0.1$ & this work \\
\hline & 2015 Oct 26 & $K_{c}$ & $786.3 \pm 1.5$ & $108.8 \pm 0.1$ & this work \\
\hline \multirow[t]{4}{*}{ HD $43691 \mathrm{~B}$} & 2013 Dec 18 & $K_{c}$ & $4550.3 \pm 1.8$ & $40.50 \pm 0.02$ & this work \\
\hline & 2014 Dec 04 & $K_{c}$ & $4546.6 \pm 2.2$ & $40.40 \pm 0.02$ & this work \\
\hline & 2015 Oct 26 & $K_{c}$ & $4540.4 \pm 2.5$ & $40.27 \pm 0.03$ & this work \\
\hline & 2016 Sep 13 & $K_{c}$ & $4536.5 \pm 2.9$ & $39.91 \pm 0.04$ & this work \\
\hline \multirow[t]{4}{*}{ HD $43691 \mathrm{C}$} & 2013 Dec 18 & $K_{c}$ & $4452.6 \pm 1.8$ & $41.08 \pm 0.02$ & this work \\
\hline & 2014 Dec 04 & $K_{c}$ & $4456.8 \pm 1.9$ & $41.08 \pm 0.02$ & this work \\
\hline & 2015 Oct 26 & $K_{c}$ & $4462.2 \pm 2.5$ & $41.08 \pm 0.03$ & this work \\
\hline & 2016 Sep 13 & $K_{c}$ & $4463.5 \pm 2.5$ & $40.90 \pm 0.03$ & this work \\
\hline \multirow[t]{5}{*}{ HD-43691 BC } & 2015 Mar 10 & $i^{\prime}$ & $4435 \pm 16^{\mathrm{b}}$ & $40.8 \pm 0.2^{\mathrm{b}}$ & Ginski et al. (2016) \\
\hline & 2013 Dec 18 & $K_{c}$ & $4513.5 \pm 1.9$ & $40.72 \pm 0.02$ & this work \\
\hline & 2014 Dec 04 & $K_{c}$ & $4512.7 \pm 2.3$ & $40.65 \pm 0.02$ & this work \\
\hline & 2015 Oct 26 & $K_{c}$ & $4510.9 \pm 2.7$ & $40.57 \pm 0.03$ & this work \\
\hline & 2016 Sep 13 & $K_{c}$ & $4508.9 \pm 3.0$ & $40.28 \pm 0.05$ & this work \\
\hline \multirow[t]{2}{*}{ HD 86081 B } & 2013 Dec 18 & $K_{c}$ & $2904.8 \pm 2.3$ & $89.29 \pm 0.06$ & this work \\
\hline & 2014 Dec 05 & $K_{c}$ & $2901.3 \pm 2.9$ & $89.35 \pm 0.06$ & this work \\
\hline \multirow[t]{3}{*}{ HD 96167 B } & 2013 Jan 24 & $K_{s}$ & $5873.0 \pm 1.8$ & $297.1 \pm 0.1$ & Mugrauer \& Ginski (2015) \\
\hline & 2014 Jan 12 & $K_{c}$ & $5889.7 \pm 3.4$ & $297.18 \pm 0.03$ & this work \\
\hline & 2015 Jan 09 & $K_{c}$ & $5884.1 \pm 2.1$ & $297.11 \pm 0.02$ & this work \\
\hline \multirow[t]{4}{*}{ HD 116029 B } & 2013 Jun 30 & $i^{\prime}$ & $1387.1 \pm 5.8$ & $209.1 \pm 0.3$ & Ginski et al. (2016) \\
\hline & $2013 \mathrm{Jul} 04$ & $K_{c}$ & $1391.6 \pm 1.7$ & $209.32 \pm 0.07$ & this work \\
\hline & 2014 Jun 09 & $K_{c}$ & $1393.0 \pm 1.6$ & $209.37 \pm 0.06$ & this work \\
\hline & 2015 Jan 09 & $K_{c}$ & $1391.4 \pm 1.6$ & $209.37 \pm 0.07$ & this work \\
\hline \multirow[t]{4}{*}{ HD $126614 \mathrm{~B}$} & 2009 Apr 13 & $K_{s}$ & $489.0 \pm 1.9$ & $56.1 \pm 0.3$ & Howard et al. (2010) \\
\hline & 2011 Jan 14 & $i^{\prime}$ & $499 \pm 67$ & $60.7 \pm 5.6$ & Ginski et al. (2012) \\
\hline & 2015 Jan 09 & $K_{c}$ & $486.1 \pm 1.5$ & $69.1 \pm 0.2$ & this work \\
\hline & 2015 Jun 24 & $K_{c}$ & $485.3 \pm 1.5$ & $70.4 \pm 0.2$ & this work \\
\hline \multirow[t]{4}{*}{ HD 142245 B } & $2013 \mathrm{Jul} 04$ & $K_{c}$ & $2484.7 \pm 1.6$ & $168.79 \pm 0.04$ & this work \\
\hline & 2014 Jun 09 & $K_{c}$ & $2499.5 \pm 1.9$ & $168.21 \pm 0.04$ & this work \\
\hline & 2015 Jun 24 & $K_{c}$ & $2501.3 \pm 1.8$ & $168.23 \pm 0.04$ & this work \\
\hline & 2016 Jun 09 & $K_{c}$ & $2507.5 \pm 1.8$ & $168.07 \pm 0.04$ & this work \\
\hline HD $142245 \mathrm{C}$ & $2013 \mathrm{Jul} 04$ & $K_{c}$ & $2524.7 \pm 1.6$ & $169.54 \pm 0.03$ & this work \\
\hline & 2014 Jun 09 & $K_{c}$ & $2516.7 \pm 1.7$ & $169.66 \pm 0.04$ & this work \\
\hline & 2015 Jun 24 & $K_{c}$ & $2513.2 \pm 1.8$ & $169.71 \pm 0.04$ & this work \\
\hline & 2016 Jun 09 & $K_{c}$ & $2505.9 \pm 1.9$ & $169.74 \pm 0.04$ & this work \\
\hline HD-142245 BC & 2012 Aug 31 & $K_{s}$ & $2498 \pm 6^{\mathrm{b}}$ & $169.2 \pm 0.2^{\mathrm{b}}$ & Mugrauer \& Ginski (2015) \\
\hline & 2013 Jul 24 & $K_{s}$ & $2494 \pm 6^{\mathrm{b}}$ & $169.1 \pm 0.1^{\mathrm{b}}$ & Mugrauer \& Ginski (2015) \\
\hline & $2013 \mathrm{Jul} 04$ & $K_{c}$ & $2503.3 \pm 1.6$ & $169.14 \pm 0.04$ & this work \\
\hline & 2014 Jun 09 & $K_{c}$ & $2507.4 \pm 2.0$ & $168.89 \pm 0.04$ & this work \\
\hline & 2015 Jun 24 & $K_{c}$ & $2506.7 \pm 1.8$ & $168.92 \pm 0.04$ & this work \\
\hline & 2016 Jun 09 & $K_{c}$ & $2506.5 \pm 1.9$ & $168.85 \pm 0.04$ & this work \\
\hline HD $164509 \mathrm{~B}$ & 2014 Jun 09 & $K_{c}$ & $698.8 \pm 1.5$ & $202.4 \pm 0.1$ & this work \\
\hline & 2014 Jul 22 & $880 \mathrm{~nm}$ & $697 \pm 2$ & $202.6 \pm 0.2$ & Wittrock et al. (2016) \\
\hline & 2015 Jun 24 & $K_{c}$ & $703.3 \pm 1.5$ & $202.9 \pm 0.1$ & this work \\
\hline & 2016 Jun 09 & $K_{c}$ & $707.7 \pm 1.5$ & $203.3 \pm 0.1$ & this work \\
\hline HD $177830 \mathrm{~B}$ & 2002 Jul 19 & $I$ & $1620 \pm 10$ & $84.1 \pm 1.0$ & Roberts et al. (2011) \\
\hline & 2005 May 08 & $K$ & $1640 \pm 10$ & $84.6 \pm 0.4$ & Eggenberger et al. (2007) \\
\hline & 2012 May 9 & $K_{s}$ & $1670 \pm 10$ & $84.3 \pm 0.2$ & Roberts et al. (2015) \\
\hline & 2012 Jun 12 & $Y J H$ & $1680 \pm 2$ & $86.0 \pm 0.1$ & Roberts et al. (2015) \\
\hline & 2014 May 14 & $K_{s}$ & $1670 \pm 10$ & $85.3 \pm 0.2$ & Roberts et al. (2015) \\
\hline & 2014 Jun 09 & $K_{c}$ & $1665.9 \pm 1.7$ & $84.27 \pm 0.06$ & this work \\
\hline & $2015 \mathrm{Jul} 05$ & $K_{c}$ & $1664.6 \pm 1.7$ & $84.19 \pm 0.06$ & this work \\
\hline & 2016 Jun 09 & $K_{c}$ & $1665.9 \pm 1.7$ & $84.12 \pm 0.06$ & this work \\
\hline HD 187123 bg & 2008 Jul 11 & $i^{\prime}$ & $2926 \pm 11$ & $48.1 \pm 0.3$ & Ginski et al. (2012) \\
\hline & 2009 Sep 07 & $i^{\prime}$ & $2917 \pm 13$ & $43.9 \pm 0.3$ & Ginski et al. (2012) \\
\hline & 2013 Aug 19 & $K_{c}$ & $2947.4 \pm 7.4$ & $29.5 \pm 0.2$ & this work \\
\hline & 2015 Jun 24 & $K_{c}$ & $3006.8 \pm 4.1$ & $22.73 \pm 0.05$ & this work \\
\hline HD 188015 bg & 2013 Jun 30 & $i^{\prime}$ & $4063 \pm 13$ & $113.7 \pm 0.2$ & Ginski et al. (2016) \\
\hline & 2013 Aug 19 & $K_{c}$ & $4079.2 \pm 1.8$ & $113.45 \pm 0.02$ & this work \\
\hline & 2014 Jul 12 & $K_{c}$ & $3992.2 \pm 1.7$ & $112.84 \pm 0.03$ & this work \\
\hline & 2014 Aug 20 & $i^{\prime}$ & $4006 \pm 67$ & $112.5 \pm 0.8$ & Ginski et al. (2016) \\
\hline HD 195019 B & 2013 Aug 19 & $K_{c}$ & $3416.3 \pm 1.7$ & $333.80 \pm 0.03$ & this work \\
\hline & 2014 Jun 09 & $K_{c}$ & $3406.9 \pm 1.8$ & $333.87 \pm 0.03$ & this work \\
\hline & $2014 \mathrm{Jul} 12$ & $K_{c}$ & $3403.9 \pm 1.7$ & $333.89 \pm 0.03$ & this work \\
\hline & 2015 Jun 03 & $K_{c}$ & $3391.1 \pm 2.0$ & $333.95 \pm 0.03$ & this work \\
\hline HD 207832 B & 2013 Aug 19 & $K_{c}$ & $2044.3 \pm 1.6$ & $218.24 \pm 0.04$ & this work \\
\hline & 2013 Aug 19 & $K_{s}$ & $2042.8 \pm 1.5$ & $218.23 \pm 0.05$ & this work \\
\hline & $2014 \mathrm{Jul} 12$ & $K_{c}$ & $2051.7 \pm 1.6$ & $218.25 \pm 0.04$ & this work \\
\hline
\end{tabular}


Table 4

(Continued)

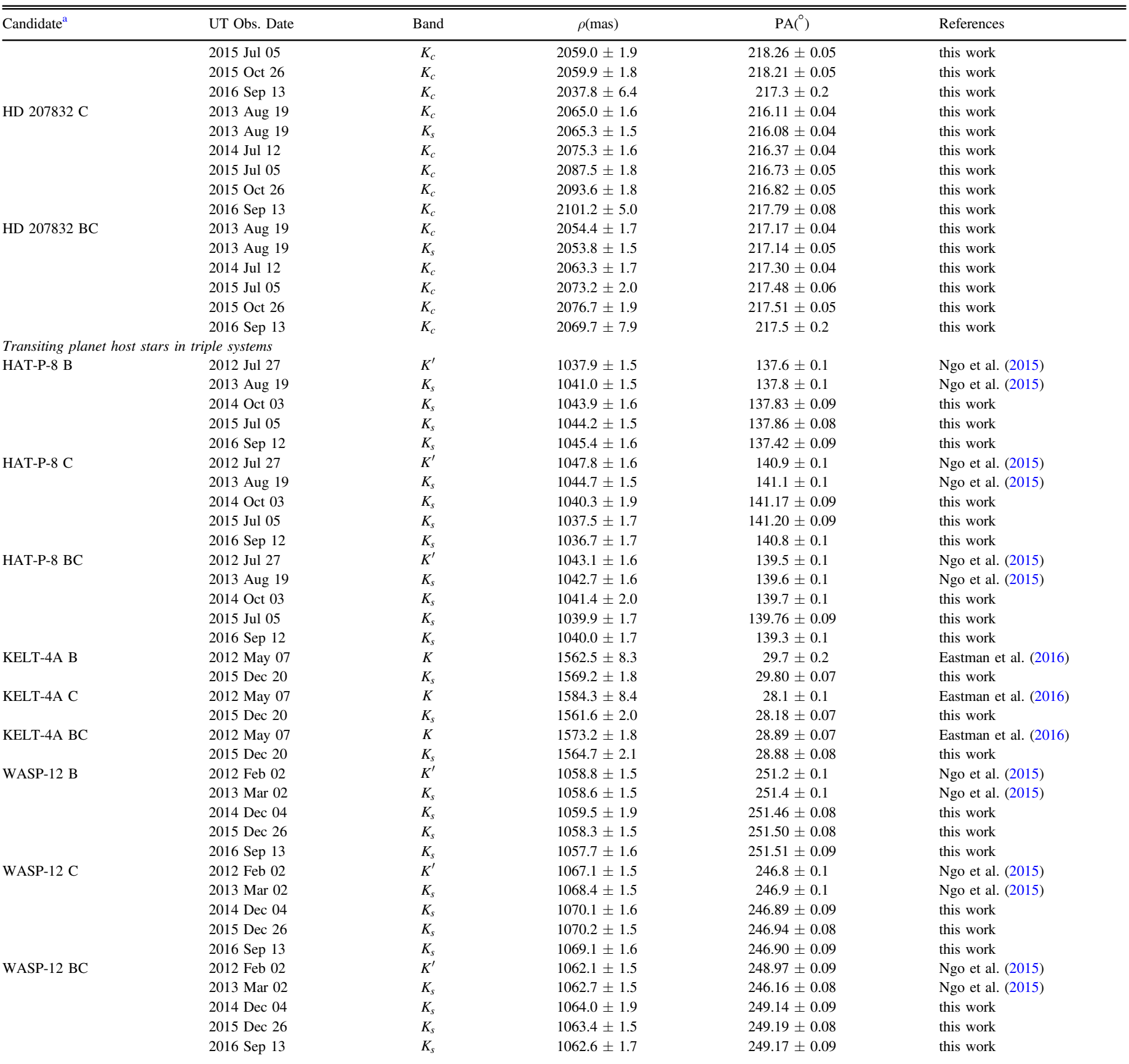

Notes. Separations $(\rho)$ and position angle (PA) measurements of candidate companions in this work and other studies with published uncertainties. For triple systems, astrometry for both individual components and their center of mass ("BC") are included. These values are also plotted in Figures 3 and 4.

${ }^{a}$ Candidate companions are labelled with uppercase letters (B, C) when our analysis determine that they are comoving stellar companions and as "bg" when they are distant background objects.

${ }^{b}$ This astrometric epoch from the literature did not resolve the individual companions, so the center of light, rather than the center of mass, is reported.

(This table is available in machine-readable form.)

consistent with all of the previous detections and also confirm that this is a comoving binary system.

$H D$ 207832. This hierarchical triple system is reported for the first time in this work. The two companions have a projected separation of 110 au from the primary star and a mutual projected separation of 4 au, making this system the most compact RV-planet-hosting triple system. Our images from 2013 through 2016 confirm that both companion stars are comoving with their host star. This system also has an extremely wide stellar companion, at 38".6 (Lodieu et al. 2014), which is far outside of our survey's field of view. With the fourth star at a projected separation of $126000 \mathrm{au}$, we 

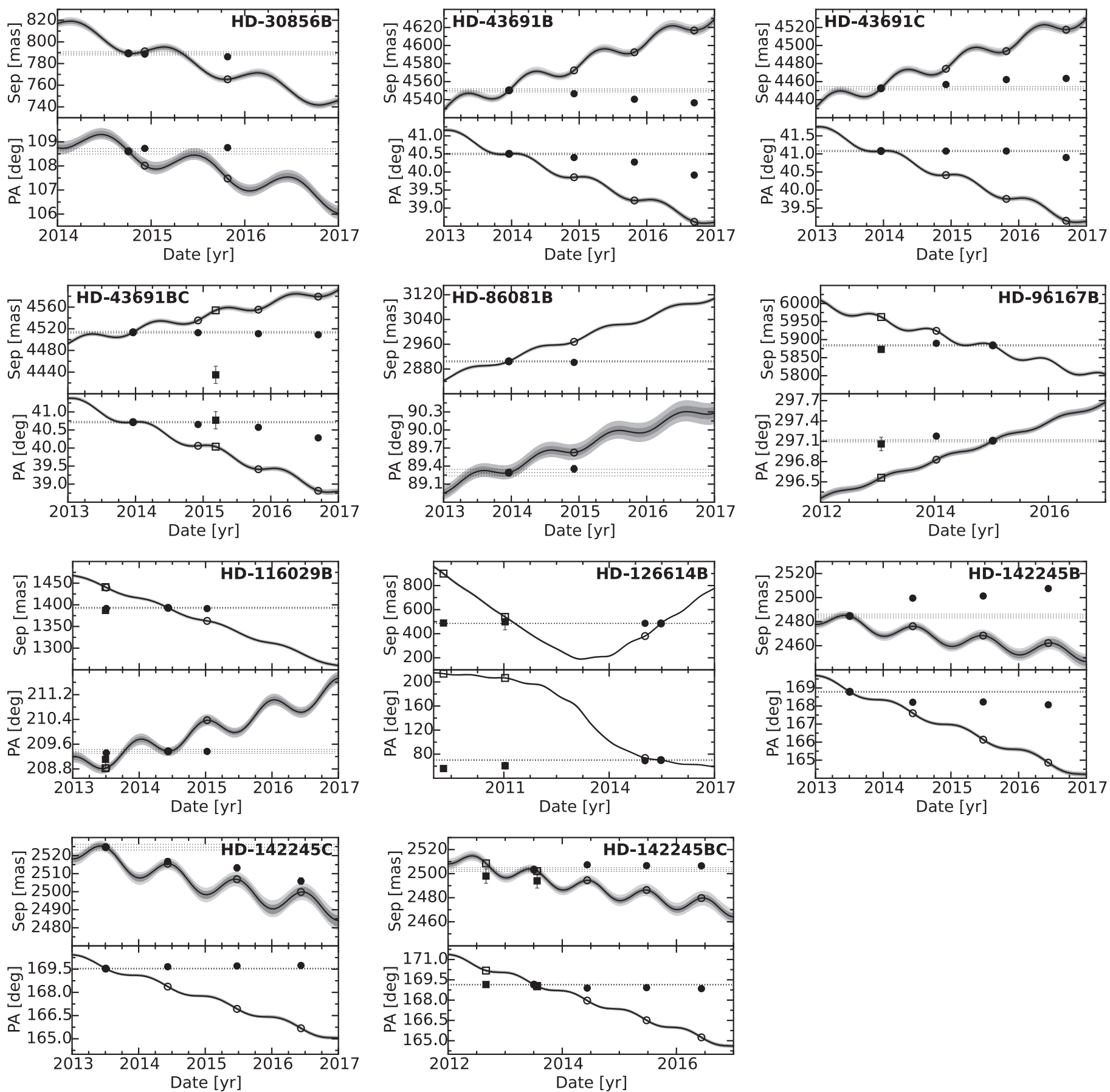

Figure 3. Top and bottom panels show the projected separation and position angle of each companion star relative to the primary star. This figure and the following figure include all confirmed common proper motion companions and background objects from our survey. The solid line shows the expected evolution of separation and position angle for an infinitely distant background object. The dark gray and light gray shaded regions represent the $68 \%$ and $95 \%$ confidence regions. We use a Monte Carlo routine accounting for uncertainties in our measurements, the primary star's celestial coordinates, proper motion, and parallax. The horizontal dashed lines represent a trajectory with no change in separation or position angle. Filled symbols show measured positions of companions while open symbols show the expected position of an object if it were a background source. Circles represent data from this work and squares represent data from the literature. The data used in this figure can be found in Table 4. Companion candidates that were determined to be physically bound are labelled as the "B" or "C" components with the center of mass of the two companions denoted as "BC."

only consider the inner hierarchical triple system for further analysis in this work. We label the primary star as "A," the northernmost companion as "B" and the other companion as "C."

Background objects. Two candidate companions were determined to be background objects rather than comoving multi-stellar systems. HD 187123 has a background object approximately $3^{\prime \prime}$ to the northeast. Ginski et al. (2012) concluded that this companion was a background star based on their 2008 and 2009 images. Our new images from 2013 and 2015 independently confirm this conclusion. We found a source approximately 4" to the northwest in our 2013 and 2014 images of HD 188015 that we determined to be a background object. Ginski et al. (2016) found two candidate companions 

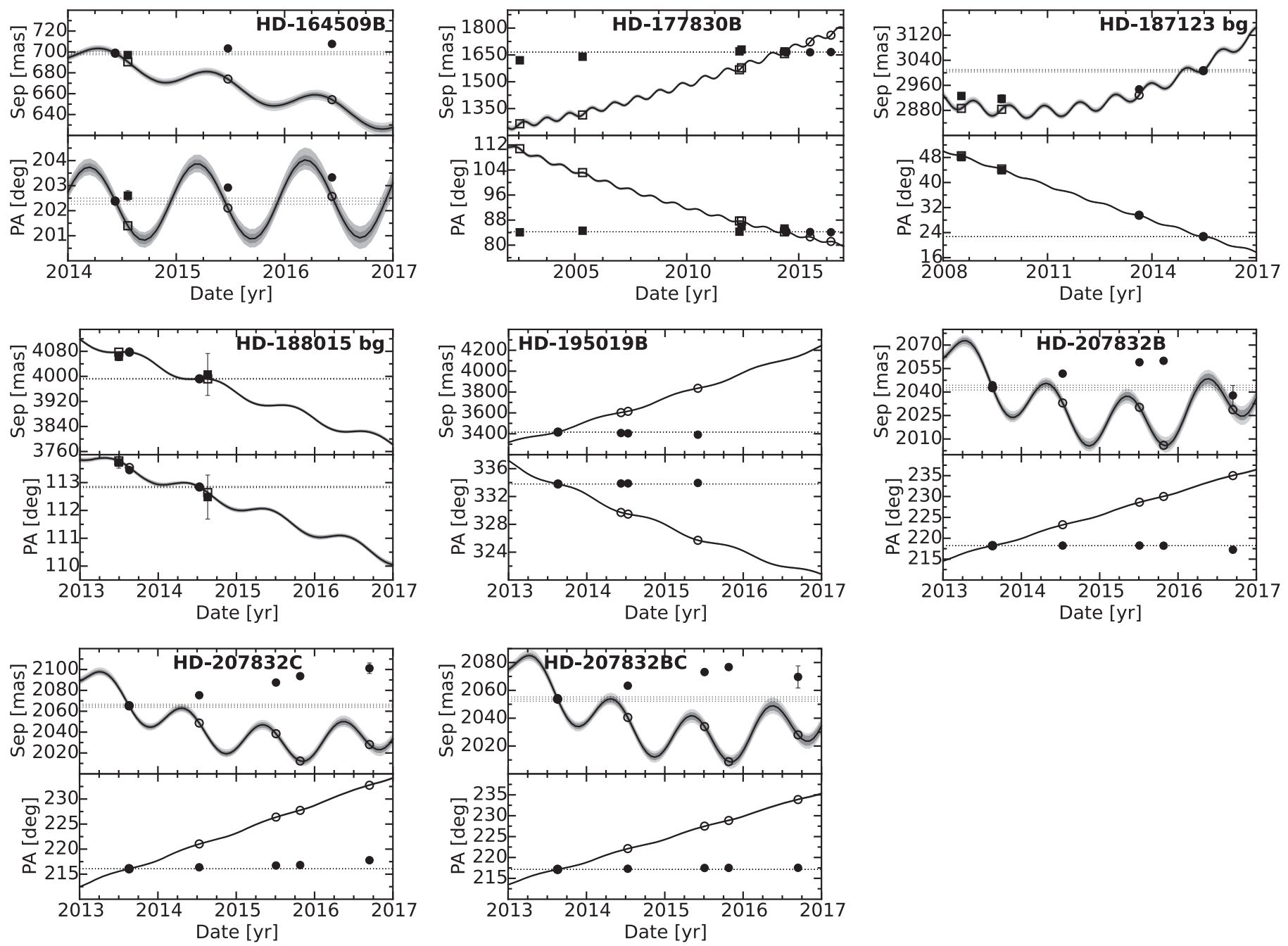

Figure 4. Continued from Figure 3. These panels also include two background objects, which are labelled as "bg."

with similar projected separations, one of which was consistent with our detection, and determined both of them to be background sources. HD 188015 does have a distant comoving companion at $11^{\prime \prime}$ (Raghavan et al. 2006), but this companion is outside our field of view and we therefore do not include it when calculating the frequency of stellar companions in our sample.

Companions beyond our survey's field of view. Six systems in our survey host companions that were outside of our survey's field of view but reported in the literature. Eggenberger et al. (2007) report companions with separations of 6!" 2 and 10!" 3 around HD 16141 and HD 46375, respectively. The Washington Double Star Catalog (WDS; Mason et al. 2001) shows that HD 109749, HD 178911, and HD 186427 (also known as 16 Cyg B) each have companions at separations of 8 !" 3,16 !" 1 , and 39 ". 6 , respectively. Finally, the WDS also reports three companions around GJ 667C at separations of 31"!2, 32!" 5, and 36!" 4.

\section{Discussion}

\subsection{Stellar Companion Fraction}

We find a raw companion fraction of 11 multi-stellar systems out of 144 surveyed stars, corresponding to a multiplicity rate of $7.6 \% \pm 2.3 \%$. For the typical target, we are sensitive to stellar-mass companions in all directions with projected separations between $0 . " 3$ and $66^{\prime \prime}$ (at 90\% directional completeness), corresponding to projected separations of $15 \mathrm{au}$ and $300 \mathrm{au}$ for a star at $50 \mathrm{pc}$. We are sensitive to companions in limited directions of up to $10^{\prime \prime}$. The most distant companion detected in our survey was found at $512 \pm 43$ au around HD 96167. It was found at the outer edge of our survey limit, at a separation of 5.9. Our raw companion fraction is consistent, within $1 \sigma$, with results from other direct imaging surveys for stellar companions around RV-detected planet host stars, as reported in Table 6. This companion fraction is lower than the Eggenberger et al. (2007) control sample's companion fraction of $17.6 \% \pm 4.9 \%$, at a significance of $1.9 \sigma$. It is not certain whether the difference is by chance, if it is due to different companion vetting by different $\mathrm{RV}$ planet surveys, or if it suggests an anti-correlation between intermediate distance giant planet and a stellar companion. Figure 5 compares the projected separations, flux ratios, and mass ratios for the companions in our survey to those reported in these other imaging surveys.

Prior to this study, there were 16 confirmed wide-binary multiple star systems with separations of less than $6^{\prime \prime}$ that were known to host RV planets. Our observations increase this number by 6 , for a total of 22 systems. This work also increases the number of confirmed multi-stellar systems with companions within 200 au by 4 ; bringing the total number of such systems to 12 . As indicated by our contrast curves, our survey 
Table 5

Derived Stellar Parameters of Confirmed Stellar Companions

\begin{tabular}{|c|c|c|c|c|c|}
\hline Companion & UT Obs. Date & $T_{\text {eff }}(\mathrm{K})$ & $M\left(M_{\odot}\right)$ & $\log g(\operatorname{cgs})$ & $D(\mathrm{au})$ \\
\hline \multicolumn{6}{|c|}{$R V$ planet host stars } \\
\hline \multirow[t]{3}{*}{ HD 30856B } & 2014 Oct 04 & $3940 \pm 200$ & $0.595 \pm 0.070$ & $4.693 \pm 0.060$ & $93.2_{-7.1}^{+8.7}$ \\
\hline & 2014 Dec 07 & $3945 \pm 70$ & $0.614 \pm 0.022$ & $4.688 \pm 0.018$ & $93.1_{-7.1}^{+8.7}$ \\
\hline & 2015 Oct $26^{*}$ & $3731 \pm 29$ & $0.537 \pm 0.013$ & $4.759 \pm 0.013$ & $92.8_{-7.1}^{+8.7}$ \\
\hline \multirow[t]{4}{*}{ HD 43691B } & 2013 Dec 18 & $2750 \pm 150$ & $0.0964 \pm 0.0091$ & $5.265 \pm 0.036$ & $366_{-23}^{+26}$ \\
\hline & 2014 Dec 04 & $2900 \pm 130$ & $0.108 \pm 0.013$ & $5.225 \pm 0.039$ & $366_{-23}^{+26}$ \\
\hline & 2015 Oct 26 & $2940 \pm 120$ & $0.113 \pm 0.015$ & $5.211 \pm 0.041$ & $365_{-23}^{+26}$ \\
\hline & 2016 Sep $13^{*}$ & $3020 \pm 110$ & $0.126 \pm 0.019$ & $5.180 \pm 0.042$ & $365_{-23}^{+26}$ \\
\hline \multirow[t]{4}{*}{ HD 43691C } & 2013 Dec 18 & $3040 \pm 110$ & $0.128 \pm 0.019$ & $5.174 \pm 0.041$ & $358_{-22}^{+25}$ \\
\hline & 2014 Dec 04 & $4100 \pm 1200$ & $0.18 \pm 0.18$ & $4.35 \pm 0.44$ & $358_{-22}^{+25}$ \\
\hline & 2015 Oct 26 & $3162 \pm 95$ & $0.153 \pm 0.025$ & $5.128 \pm 0.039$ & $359_{-22}^{+25}$ \\
\hline & 2016 Sep $13^{*}$ & $3308 \pm 71$ & $0.209 \pm 0.037$ & $5.056 \pm 0.042$ & $359_{-22}^{+25}$ \\
\hline \multirow[t]{2}{*}{ HD 86081B } & 2013 Dec 18 & $2400 \pm 1700$ & $0.08 \pm 0.54$ & $5.34 \pm 0.63$ & $277_{-24}^{+29}$ \\
\hline & 2014 Dec $05^{*}$ & $2562 \pm 61$ & $0.0876 \pm 0.0019$ & $5.305 \pm 0.010$ & $276_{-24}^{+29}$ \\
\hline \multirow[t]{2}{*}{ HD 96167B } & 2014 Jan $12^{*}$ & $3080 \pm 140$ & $0.135 \pm 0.028$ & $5.159 \pm 0.053$ & $512_{-37}^{+43}$ \\
\hline & 2015 Jan 09 & $2820 \pm 240$ & $0.102 \pm 0.025$ & $5.224 \pm 0.081$ & $512_{-37}^{+43}$ \\
\hline \multirow[t]{3}{*}{ HD 116029B } & 2013 Jul 04* & $3387 \pm 18$ & $0.259 \pm 0.014$ & $5.004 \pm 0.014$ & $171_{-13}^{+15}$ \\
\hline & 2014 Jun 09 & $3378 \pm 21$ & $0.252 \pm 0.015$ & $5.010 \pm 0.015$ & $171_{-13}^{+15}$ \\
\hline & 2015 Jan 09 & $3343 \pm 29$ & $0.229 \pm 0.017$ & $5.033 \pm 0.019$ & $171_{-13}^{+15}$ \\
\hline \multirow[t]{2}{*}{ HD 126614B } & 2015 Jan 09* & $3382 \pm 23$ & $0.255 \pm 0.017$ & $5.008 \pm 0.016$ & $35.3_{-2.3}^{+2.6}$ \\
\hline & 2015 Jun 24 & $3434 \pm 28$ & $0.300 \pm 0.028$ & $4.966 \pm 0.023$ & $35.2_{-2.3}^{+2.6}$ \\
\hline \multirow[t]{4}{*}{ HD 142245B } & $2013 \mathrm{Jul} 04^{*}$ & $3589 \pm 14$ & $0.455 \pm 0.011$ & $4.842 \pm 0.011$ & $273_{-17}^{+20}$ \\
\hline & 2014 Jun 09 & $3824 \pm 63$ & $0.573 \pm 0.025$ & $4.723 \pm 0.022$ & $275_{-17}^{+20}$ \\
\hline & 2015 Jun 24 & $3651 \pm 20$ & $0.501 \pm 0.011$ & $4.796 \pm 0.011$ & $275_{-18}^{+20}$ \\
\hline & 2016 Jun 09 & $3628 \pm 14$ & $0.484 \pm 0.011$ & $4.812 \pm 0.010$ & $276_{-18}^{+20}$ \\
\hline \multirow[t]{4}{*}{ HD $142245 C$} & $2013 \mathrm{Jul} \mathrm{04*}$ & $3687 \pm 20$ & $0.5172 \pm 0.0091$ & $4.7791 \pm 0.0094$ & $278_{-18}^{+20}$ \\
\hline & 2014 Jun 09 & $3847 \pm 80$ & $0.580 \pm 0.030$ & $4.717 \pm 0.027$ & $277_{-18}^{+20}$ \\
\hline & 2015 Jun 24 & $3725 \pm 20$ & $0.5343 \pm 0.0090$ & $4.7619 \pm 0.0088$ & $276_{-18}^{+20}$ \\
\hline & 2016 Jun 09 & $3741 \pm 21$ & $0.5417 \pm 0.0097$ & $4.7547 \pm 0.0094$ & $276_{-18}^{+20}$ \\
\hline \multirow[t]{3}{*}{ HD 164509B } & 2014 Jun 09 & $3403 \pm 40$ & $0.268 \pm 0.035$ & $4.990 \pm 0.034$ & $36.6_{-1.7}^{+2.0}$ \\
\hline & 2015 Jun $24^{*}$ & $3480 \pm 14$ & $0.355 \pm 0.015$ & $4.924 \pm 0.013$ & $36.9_{-1.8}^{+2.0}$ \\
\hline & 2016 Jun 09 & $3515 \pm 29$ & $0.388 \pm 0.029$ & $4.895 \pm 0.023$ & $37.1_{-1.8}^{+2.0}$ \\
\hline \multirow[t]{3}{*}{ HD 177830B } & 2014 Jun 09 & $3159 \pm 33$ & $0.1524 \pm 0.0087$ & $5.129 \pm 0.014$ & $98.3_{-3.5}^{+3.8}$ \\
\hline & 2015 Jul 05 & $3139 \pm 42$ & $0.1475 \pm 0.0099$ & $5.137 \pm 0.016$ & $98.2_{-3.5}^{+3.8}$ \\
\hline & 2016 Jun $09^{*}$ & $3260 \pm 33$ & $0.185 \pm 0.014$ & $5.083 \pm 0.017$ & $98.3_{-3.5}^{+3.8}$ \\
\hline \multirow[t]{4}{*}{ HD 195019B } & 2013 Aug 19 & $4000 \pm 120$ & $0.631 \pm 0.036$ & $4.673 \pm 0.031$ & $131.5_{-4.8}^{+5.1}$ \\
\hline & 2014 Jun $09^{*}$ & $3890 \pm 100$ & $0.599 \pm 0.034$ & $4.702 \pm 0.030$ & $131.2_{-4.8}^{+5.1}$ \\
\hline & 2014 Jul 12 & $4000 \pm 130$ & $0.632 \pm 0.038$ & $4.672 \pm 0.032$ & $131.1_{-4.8}^{+5.1}$ \\
\hline & 2015 Jun 03 & $3940 \pm 110$ & $0.614 \pm 0.033$ & $4.689 \pm 0.029$ & $130.6_{-4.7}^{+5.1}$ \\
\hline \multirow[t]{5}{*}{ HD 207832B } & 2013 Aug 19 & $2680 \pm 140$ & $0.0931 \pm 0.0070$ & $5.278 \pm 0.030$ & $111.2_{-5.3}^{+5.9}$ \\
\hline & 2014 Jul 12 & $2716 \pm 90$ & $0.0944 \pm 0.0051$ & $5.273 \pm 0.021$ & $111.6_{-5.3}^{+6.0}$ \\
\hline & $2015 \mathrm{Jul} 05^{*}$ & $2870 \pm 81$ & $0.1053 \pm 0.0076$ & $5.234 \pm 0.024$ & $112.0_{-5.4}^{+6.0}$ \\
\hline & 2015 Oct 26 & $2819 \pm 86$ & $0.1007 \pm 0.0065$ & $5.249 \pm 0.023$ & $112.1_{-5.4}^{+6.0}$ \\
\hline & 2016 Sep 13 & $2623 \pm 100$ & $0.0894 \pm 0.0040$ & $5.295 \pm 0.020$ & $110.9_{-5.3}^{+5.9}$ \\
\hline \multirow[t]{5}{*}{ HD $207832 C$} & 2013 Aug 19 & $2864 \pm 98$ & $0.1049 \pm 0.0094$ & $5.234 \pm 0.030$ & $112.3_{-5.4}^{+6.0}$ \\
\hline & $2014 \mathrm{Jul} 12$ & $2838 \pm 85$ & $0.1023 \pm 0.0071$ & $5.243 \pm 0.024$ & $112.9_{-5.4}^{+6.0}$ \\
\hline & $2015 \mathrm{Jul} 05^{*}$ & $2855 \pm 80$ & $0.1040 \pm 0.0069$ & $5.238 \pm 0.023$ & $113.6_{-5.4}^{+6.1}$ \\
\hline & 2015 Oct 26 & $2850 \pm 80$ & $0.1035 \pm 0.0067$ & $5.240 \pm 0.022$ & $113.9_{-5.4}^{+6.4}$ \\
\hline & 2016 Sep 13 & $2958 \pm 75$ & $0.116 \pm 0.010$ & $5.205 \pm 0.027$ & $114.3_{-5.5}^{+6.1}$ \\
\hline \multicolumn{6}{|c|}{ Transiting planet host stars in triple systems } \\
\hline HAT-P-8B & 2014 Oct 03 & $3186 \pm 26$ & $0.1604 \pm 0.0076$ & $5.118 \pm 0.011$ & $238 \pm 13$ \\
\hline & 2015 Jul 07 & $3205 \pm 23$ & $0.1659 \pm 0.0068$ & $5.1099 \pm 0.0097$ & $238 \pm 13$ \\
\hline & 2016 Sep $12^{*}$ & $3206 \pm 22$ & $0.1664 \pm 0.0065$ & $5.1092 \pm 0.0093$ & $238 \pm 13$ \\
\hline HAT-P-8C & 2014 Oct 03 & $3100 \pm 31$ & $0.1393 \pm 0.0065$ & $5.152 \pm 0.012$ & $237 \pm 13$ \\
\hline & 2015 Jul 07 & $3022 \pm 21$ & $0.1250 \pm 0.0031$ & $5.1810 \pm 0.0076$ & $236 \pm 13$ \\
\hline & 2016 Sep $12^{*}$ & $3024 \pm 21$ & $0.1254 \pm 0.0031$ & $5.1801 \pm 0.0076$ & $236 \pm 13$ \\
\hline KELT-4A B & 2015 Dec $20^{*}$ & $4560 \pm 320$ & $0.776 \pm 0.081$ & $4.565 \pm 0.066$ & $331_{-19}^{+20}$ \\
\hline KELT-4A C & 2015 Dec $20^{*}$ & $3846 \pm 83$ & $0.584 \pm 0.029$ & $4.715 \pm 0.026$ & $330_{-19}^{+20}$ \\
\hline WASP-12B & 2014 Dec 04 & $3861 \pm 66$ & $0.589 \pm 0.022$ & $4.711 \pm 0.019$ & $462 \pm 40$ \\
\hline
\end{tabular}


Table 5

(Continued)

\begin{tabular}{|c|c|c|c|c|c|}
\hline Companion & UT Obs. Date & $T_{\text {eff }}(\mathrm{K})$ & $M\left(M_{\odot}\right)$ & $\log g(\mathrm{cgs})$ & $D(\mathrm{au})$ \\
\hline & 2016 Sep 13 & $3842 \pm 55$ & $0.583 \pm 0.019$ & $4.716 \pm 0.017$ & $461 \pm 39$ \\
\hline WASP-12C & 2014 Dec 04 & $3690 \pm 53$ & $0.518 \pm 0.024$ & $4.778 \pm 0.024$ & $467 \pm 40$ \\
\hline & 2016 Sep 13 & $3839 \pm 56$ & $0.582 \pm 0.020$ & $4.717 \pm 0.018$ & $466 \pm 40$ \\
\hline
\end{tabular}

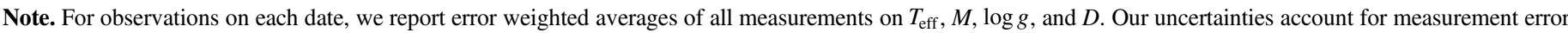

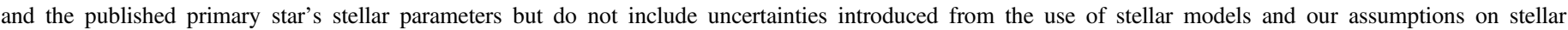

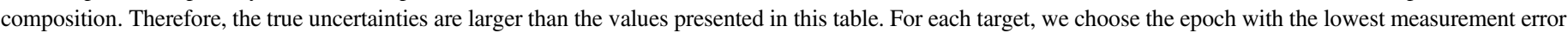
as the representative value for further dynamical analysis. This epoch is marked with an asterisk.

(This table is available in machine-readable form.)
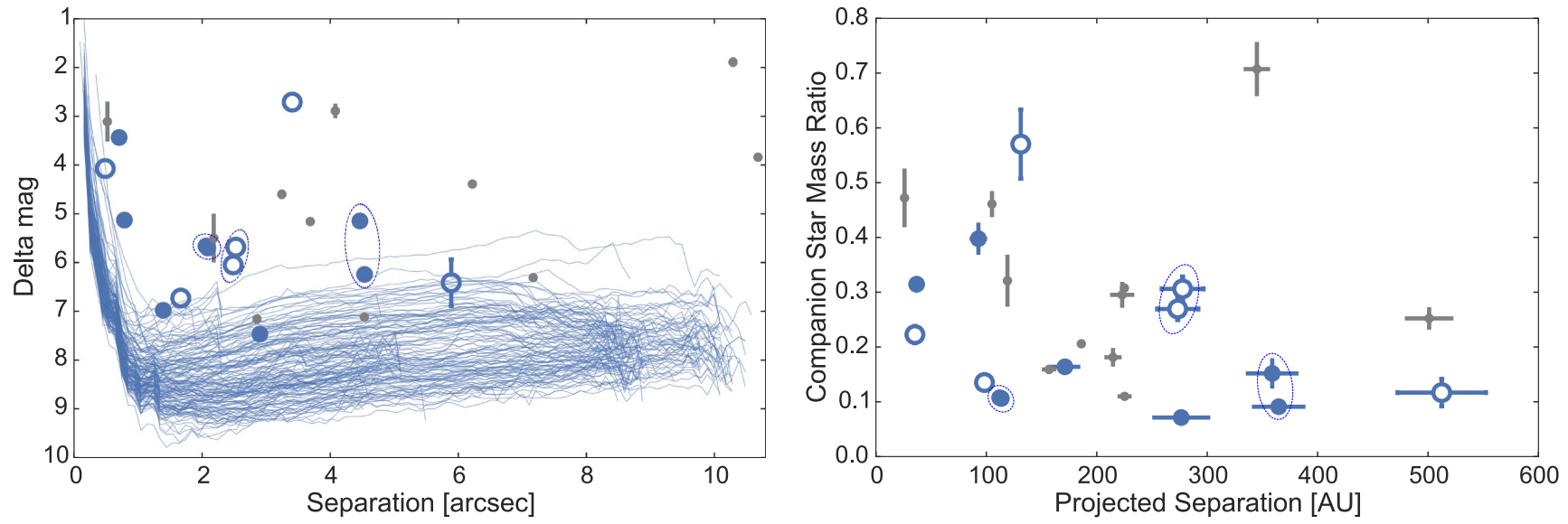

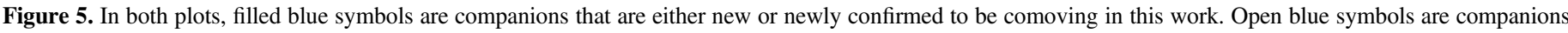

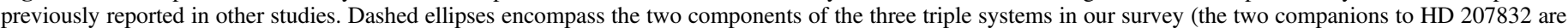

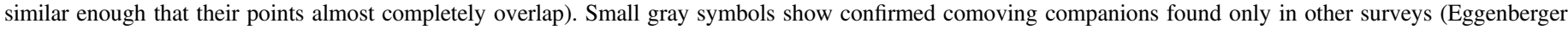

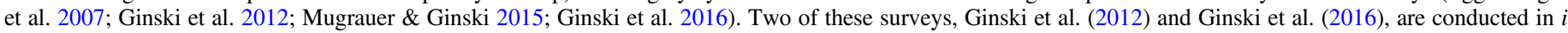

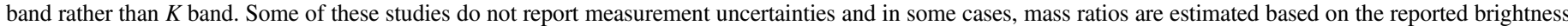

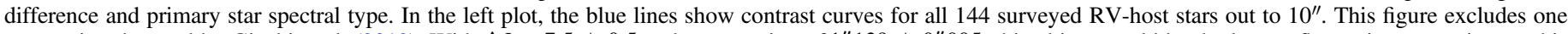

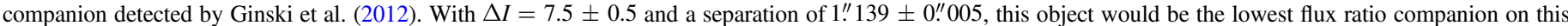
plot. However, the target star is HD 176051, which is a previously known binary hosting an astrometry detected planet, not an RV-detected planet.

is more sensitive at small separations than these previous surveys. Figure 5 shows that the majority of our new confirmed multi-stellar systems have relatively small flux ratios and projected separations as compared to the sample of previously published planet-hosting multiple star systems. All known multi-stellar RV-planet hosting systems with companions within $6 "$ are listed in Table 7 . For each system, we calculate $\nu / n$, the ratio of the planet's precession due to the companion star divided by the planet's mean motion as a proxy for the companion star's ability to dynamically influence the planet. We sort the multi-stellar systems by this metric in order to highlight the most interesting systems for future dynamical studies.

The stellar companion rate for our population of RVdetected giant planet host stars is much lower than the companion fraction of $47 \% \pm 7 \%$ that we reported for transiting hot-Jupiter systems (Ngo et al. 2016). This is most likely due to the relatively severe biases against multiple star systems in the target selection process for RV surveys. Unfortunately, these biases are neither well characterized nor fully reported, and we are therefore unable to report a completeness corrected stellar multiplicity rate for the RVdetected planet population. Unlike transit surveys, RV surveys such as the California Planet Survey (Howard et al. 2010) and the HARPS survey (Lagrange et al. 2009), vet potential targets for known companion stars that are close enough (generally within $2^{\prime \prime}$ ) to fall within the spectrograph slit and are bright enough to have detectable spectral lines at the optical wavelengths where most RV surveys operate. Although it is possible to measure RV shifts for double-lined spectroscopic binaries, RV pipelines developed to search for planets are not typically designed to accommodate a second set of spectral lines and therefore avoid these kinds of systems. Nearby stars are generally identified via archival surveys such as the Washington Double Star catalog (Mason et al. 2001). RV surveys also discard targets that show large RV variations, effectively eliminating close binaries from their samples. Although RV-planet survey target selection is performed with some quantitative and objective metrics, there are also any number of subjective choices made over the years that are difficult to quantify retroactively (Clanton \& Gaudi 2014). We therefore conclude that we cannot reliably compare stellar 
Table 6

Stellar Companion Fraction of Stars in RV-planet Surveys

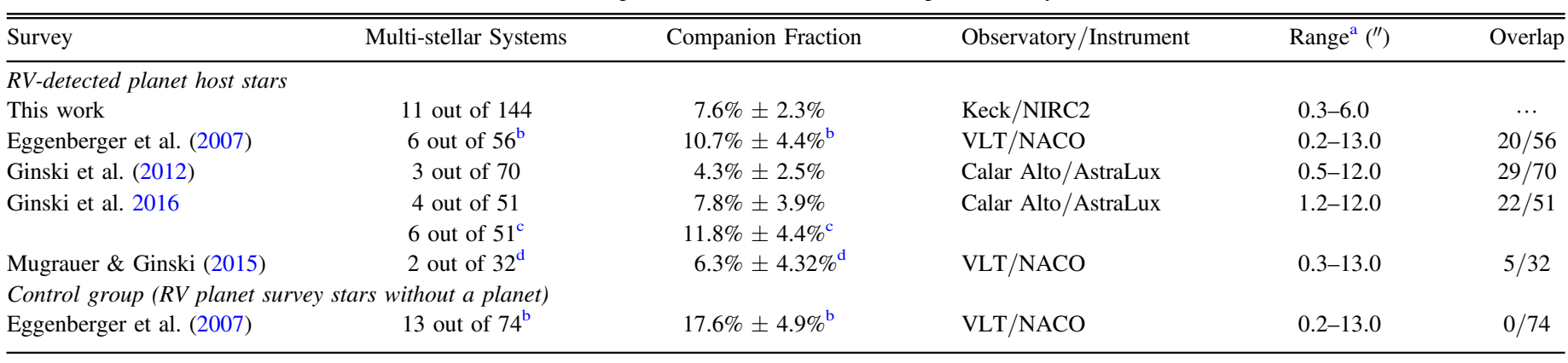

Notes. Companion fractions were computed from the raw number of confirmed multi-stellar systems reported by each survey, assuming Poisson uncertainties. These companion fractions are not corrected for survey completeness. Only stars that have RV-detected planet hosts are counted. The "overlap" column indicates the number of targets from the cited survey that are also in our survey. Out of our 144 targets, 73 have also been imaged in the other surveys; however, only 26 out of 144 targets have been previously imaged at observatories comparable to Keck.

${ }^{\mathrm{a}}$ The inner limit of the range corresponds to a separation where the survey is sensitive to a contrast of $\Delta K \sim 5$ ( $\Delta I \sim 7$ for the AstraLux surveys). We determine the outer limit of our survey to be the separation where we have $90 \%$ directional completeness; however, our full chip size is $10^{\prime \prime}$. For other surveys, we report their instrument's full chip size as the outer limit.

${ }^{\mathrm{b}}$ One target in Eggenberger et al. (2007), HD 33636, was originally in our survey list until we learned the detection of HD 33636 was retracted. Therefore, we count this system as part of Eggenberger et al. (2007)'s control group instead of their planet hosting sample.

${ }^{c}$ Ginski et al. (2016) reported four confirmed multi-stellar system and several candidate multi-stellar systems. Two of these ambiguous cases (HD 43691 and HD 116029) were also in our survey and we confirmed them to be comoving systems. In this row, we report an updated companion fraction rate with these two additional systems.

${ }^{d}$ We were unable to confirm the planetary status for the HD 9578 system surveyed by Mugrauer \& Ginski (2015) in the peer-reviewed literature. Thus, we exclude it from this count.

multiplicity rates for planet-hosting stars from RV surveys with similar results for samples of planets detected by transit surveys.

\subsection{Characteristics of $R V$-detected Planets in Multi-stellar Systems}

Although RV surveys are undoubtedly subject to different selection biases than transit surveys when it comes to multiple star systems, this bias is effectively removed when we limit ourselves to comparing different sub-samples within our RV planet survey population, assuming the selection biases affect all sub-samples in the same way. Previous studies have suggested that stellar companions are less common in systems with long-period planets than those with short-period planets (e.g Kaib et al. 2013; Zuckerman 2014; Wang et al. 2015a). Here, we compare properties of planets in multi-stellar and single-star systems within our survey population of RVdetected planet host stars. Figure 6 and Table 7 show the orbital properties of the innermost planet of each single and multistellar system in our survey. We wish to determine whether the distributions for innermost planet orbital eccentricity, orbital period, and mass differ for single-star systems from multi-star systems. Thus, we calculate whether a two-population (for single and multi-stellar systems) distribution is a better fit to the data than a one-population model. To avoid tidal circularization effects, we exclude 35 single and 5 multi-stellar systems with planets with semimajor axes less than 0.1 au in this analysis. Because we have a relatively small sample of multiple star systems in our survey, we also include similar multiple star systems (i.e., those with projected separations less than 6"; see Table 7 for a complete list) from the published literature. In total, there are 98 single-star systems and 17 multi-stellar systems considered in this part of the analysis.
Assuming planet eccentricities follow a beta distribution (Kipping 2013), we calculate the probability of obtaining an individual planet orbital eccentricity $e_{k}$ to be

$$
\operatorname{prob}\left(e_{k} \mid a, b\right)=\frac{\Gamma(a+b)}{\Gamma(a) \Gamma(b)} e^{a-1}(1-e)^{b-1},
$$

where $\Gamma()$ denotes the Gamma function and $a$ and $b$ are the model parameters. We assume the planet mass and orbital period take the form of the Cumming et al. (2008) power law, so that the probability of obtaining an individual planet mass $m_{k}$ and orbital period $P_{k}$ is

$$
\operatorname{prob}\left(m_{k}, P_{k} \mid \alpha, \beta\right) \propto m^{\alpha} P^{\beta},
$$

where $\alpha$ and $\beta$ are the model parameters. We assume that the orbital eccentricity is not correlated with orbital period and planetary mass, so we can determine the probability of obtaining any individual planetary system to be the product of the above probabilities. Our goal is to compute the likelihood of a model $M$ with a set of parameters $\theta=(a, b, \alpha, \beta)$ for a set of planets. For an individual system, we can write

$$
\operatorname{prob}\left(e_{k}, m_{k}, P_{k} \mid \theta, M\right) \propto \frac{\Gamma(a+b)}{\Gamma(a) \Gamma(b)} e^{a-1}(1-e)^{b-1} m^{\alpha} P^{\beta} .
$$

From Bayes' Theorem and choosing uniform priors for all model parameters, we can write the log-likelihood of a onepopulation model $\mathcal{L}_{1}$ as

$$
\mathcal{L}_{1}=\sum_{k} \ln \left[\operatorname{prob}\left(e_{k}, m_{k}, P_{k} \mid M, \theta\right)\right]+C,
$$

where the sum over $k$ includes all RV planet host systems and $C$ is a constant. Similarly, we can write the log-likelihood of a 

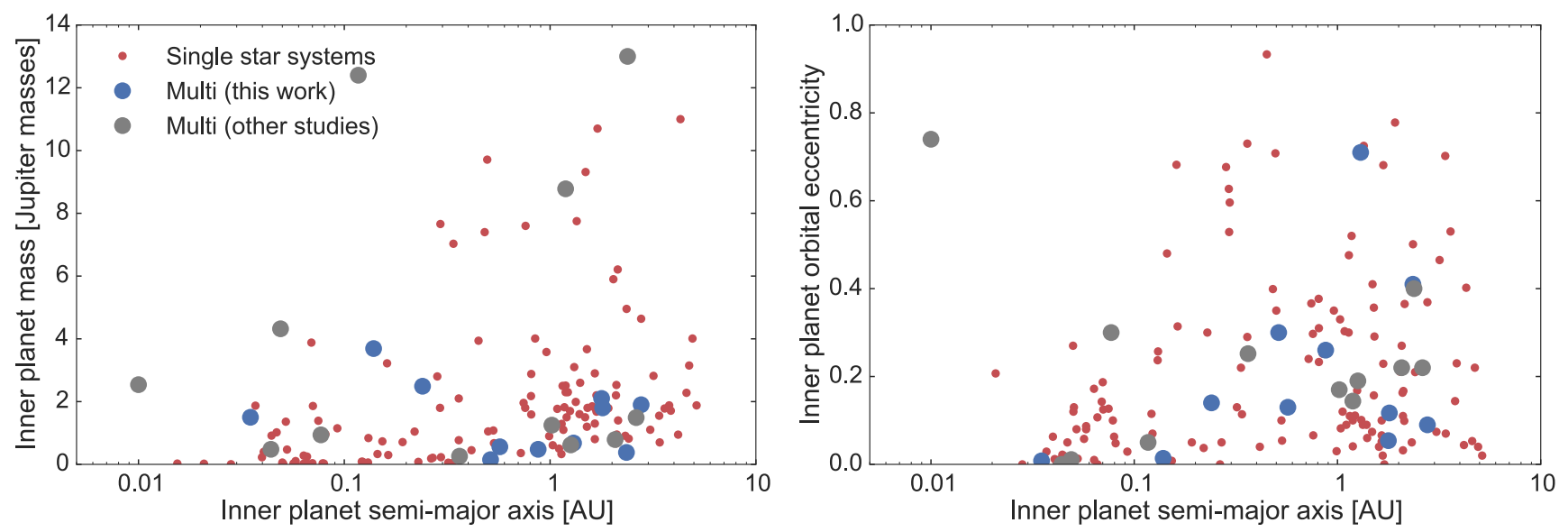

Figure 6. Mass and eccentricity vs. semimajor axis of the innermost planet for single-star systems (red) and multi-stellar systems from our survey (blue) and other studies listed in Table 7 (gray).

Table 7

Stellar and Planetary Parameters of RV-planet Host Systems with Stellar Companions within 6"

\begin{tabular}{|c|c|c|c|c|c|c|c|c|c|c|c|}
\hline \multirow[b]{2}{*}{ Planet Host } & $M_{\text {host }}$ & $a_{\mathrm{pl}}$ & \multirow[b]{2}{*}{$e_{\mathrm{pl}}$} & \multirow{2}{*}{$\begin{array}{c}m_{\mathrm{pl}} \\
\left(M_{\mathrm{Jup}}\right)\end{array}$} & \multirow[b]{2}{*}{ References } & \multirow{2}{*}{$\begin{array}{l}M_{\text {comp }} \\
\left(M_{\odot}\right)\end{array}$} & \multirow{2}{*}{$\begin{array}{l}a_{\text {comp }} \\
(\mathrm{au})\end{array}$} & \multirow{2}{*}{$\begin{array}{c}\rho_{\text {comp }} \\
\left(^{\prime \prime}\right)\end{array}$} & \multirow[b]{2}{*}{ References } & \multirow{2}{*}{\multicolumn{2}{|c|}{$\nu / n$}} \\
\hline & $\left(M_{\odot}\right)$ & (au) & & & & & & & & & \\
\hline HD 164509 & $1.13(2)$ & $0.875(8)$ & $0.3(1)$ & $0.48(9)$ & Gig12 & $0.36(2)$ & $37(2)$ & $0.703(2)$ & this work & 4 & $\times 10^{-6}$ \\
\hline HD 197037 & $1.06(2)$ & $2.07(5)$ & $0.22(7)$ & $0.79(5)$ & $\mathrm{S} 15, \mathrm{R} 12$ & $0.34(5)$ & $119(2)$ & $3.688(9)$ & G16 & 2 & $\times 10^{-6}$ \\
\hline HD 217786 & $1.02(3)$ & $2.38(4)$ & $0.40(5)$ & $13.0(8)$ & Mo11 & $0.162(7)$ & $157(7)$ & $2.856(7)$ & G16 & 6 & $\times 10^{-7}$ \\
\hline HD $142245^{\mathrm{a}}$ & $1.7(1)$ & 2.77 (9) & 0.09() & $1.90(2)$ & J11 & $0.97(1)$ & $276(14)$ & 2.505 & this work & 6 & $\times 10^{-7}$ \\
\hline HD $132563 B^{a}$ & $1.01(1)$ & $2.62(4)$ & $0.22(9)$ & $1.49(9)$ & D11 & $1.64(2)$ & $400(50)$ & $4.11(2)$ & D11 & 5 & $\times 10^{-7}$ \\
\hline HD 116029 & $1.6(1)$ & $1.78(5)$ & 0.054() & $2.1(2)$ & J11 & $0.26(1)$ & $171(15)$ & $1.392(1)$ & this work & 2 & $\times 10^{-7}$ \\
\hline HD 87646 & $1.12(9)$ & $0.117(3)$ & $0.05(2)$ & $12.4(7)$ & M16 & $0.6^{\mathrm{c}}$ & $15.7(2.1)$ & 0.213() & M16, HIP & 2 & $\times 10^{-7}$ \\
\hline HD 89484 & $1.2(2)$ & $1.19(2)$ & $0.14(5)$ & $9(1)$ & Ha10 & $0.9^{\mathrm{c}}$ & $178(6)$ & 4.629() & $\mathrm{Ma06}$ & 2 & $\times 10^{-7}$ \\
\hline HD $207832^{\mathrm{a}, \mathrm{d}}$ & $0.98(7)$ & $0.570(2)$ & $0.13_{-0.05}^{+0.18}$ & $0.56_{-0.03}^{+0.06}$ & $\mathrm{~S} 13, \mathrm{H} 12$ & $0.21(1)$ & $113(4)$ & $2.073(2)$ & this work & 3 & $\times 10^{-8}$ \\
\hline HD $177830^{\mathrm{e}}$ & $1.37(4)$ & $0.5137(3)$ & $0.3(1)$ & $0.15(2)$ & J06,Me11 & $0.19(1)$ & $98(4)$ & $1.666(2)$ & this work & 2 & $\times 10^{-8}$ \\
\hline HD 86081 & $1.23(8)$ & $0.035^{\mathrm{f}}()$ & 0.008 (4) & 1.5() & S13,J06 & $0.088(2)$ & $276(29)$ & $2.901(2)$ & this work & 1 & $\times 10^{-10}$ \\
\hline HD $43691^{\mathrm{a}}$ & $1.38(5)$ & 0.24() & $0.14(2)$ & 2.49() & ds07 & $0.33(4)$ & $362(18)$ & $4.500(2)$ & this work & 7 & $\times 10^{-11}$ \\
\hline HD 41004A & $0.7^{\mathrm{c}}$ & $0.006^{\mathrm{f}}(2)$ & $0.7(2)$ & $2.5(7)$ & S02,Z04 & $0.4^{\mathrm{c}}$ & 22.5() & 0.5() & S02,HIP & 1 & $\times 10^{-11}$ \\
\hline HD 185269 & $1.3(1)$ & $0.077^{\mathrm{f}}()$ & $0.23(3)$ & $1.03(3)$ & Mo06 & 0.23 & $215(8)$ & $4.53(1)$ & G16 & 8 & $\times 10^{-12}$ \\
\hline tau Boo & $1.34(5)$ & $0.049^{\mathrm{f}}(3)$ & $0.011(6)$ & $4.32(4)$ & B12,B15 & 0.4() & 225 (1) & 2.18 (1) & Gin12 & 3 & $\times 10^{-12}$ \\
\hline
\end{tabular}

Notes. All RV-planet hosting systems with stellar companions detected within $6^{\prime \prime}$. As this is a soft limit (see the text), we included HD 16141 with a companion at 6". 22. For brevity, the number(s) in parentheses are uncertainties on the last digit(s) (i.e., 2.35 (2) is $2.35 \pm 0.02$ ). Missing values indicate no uncertainty provided by the source. References for the system and companion parameters follow each set of columns. We rank this list by a metric, $\nu / n=\frac{M_{\mathrm{comp}}}{M_{\mathrm{host}}}\left(\frac{a_{\mathrm{pl}}}{a_{\mathrm{comp}}}\right)^{3}$, to represent strength of the precession induced on the planet by the companion star. Systems with new or newly confirmed stellar companions have boldface names and companion properties.

a This is a triple system. The reported $M_{\text {comp }}$ is the combined mass of both stars and $a_{\text {comp }}$ is the average projected separation of each component.

${ }^{\mathrm{b}}$ The innermost planet, HD 142b, is reported here. This system also hosts a second planet, HD $142 \mathrm{c}$, at 6.8 au and $5.3 M_{\text {jup. }}$.

c These stellar masses were not measured. Instead, they were estimated from the star's spectral type.

d The innermost planet, HD 207832b, is reported here. This system also hosts a second planet, HD 207832c, at 2.112 au and $0.73 M_{\text {jup. }}$.

e The innermost planet, HD $177830 \mathrm{c}$, is reported here. This system also hosts a second planet, HD $177830 \mathrm{~b}$, at 1.2218 au and $1.49 M_{\text {jup }}$.

${ }^{\mathrm{f}}$ This system is not included in the comparison between single and multi-stellar systems described in Section 5.2 because the planet's semimajor axis is less than 0.1 au.

References. (B06) Butler et al. (2006), (B12) Brogi et al. (2012), (B15) Borsa et al. (2015), (D11) Desidera et al. (2011), (E07) Eggenberger et al. (2007), (G10) Ghezzi et al. (2010), (Gig12) Giguere et al. (2012), (Gin12) Ginski et al. (2012), (G16) Ginski et al. (2016), (Ha10) Han et al. (2010), (Ho10) Howard et al. (2010), (H12) Haghighipour et al. (2012), (HIP) Hipparcos Catalogue Perryman et al. (1997), (J06) Johnson et al. (2006), (J11) Johnson et al. (2011), (J15) Jofré et al. (2015), (M05) Moutou et al. (2005), (Ma06) Mason et al. (2004), (Mo06) Moutou et al. (2006), (Me11) Meschiari et al. (2011), (Mo11) Moutou et al. (2011), (M16) Ma et al. (2016), (P09) Peek et al. (2009), (S02) Santos et al. (2002), (dS07) Da Silva et al. (2007), (S13) Santos et al. (2013), (S15) Sousa et al. (2015), (W12) Wittenmyer et al. (2012), (W13) Wittenmyer et al. (2013), (Z04) Zucker et al. (2004). 


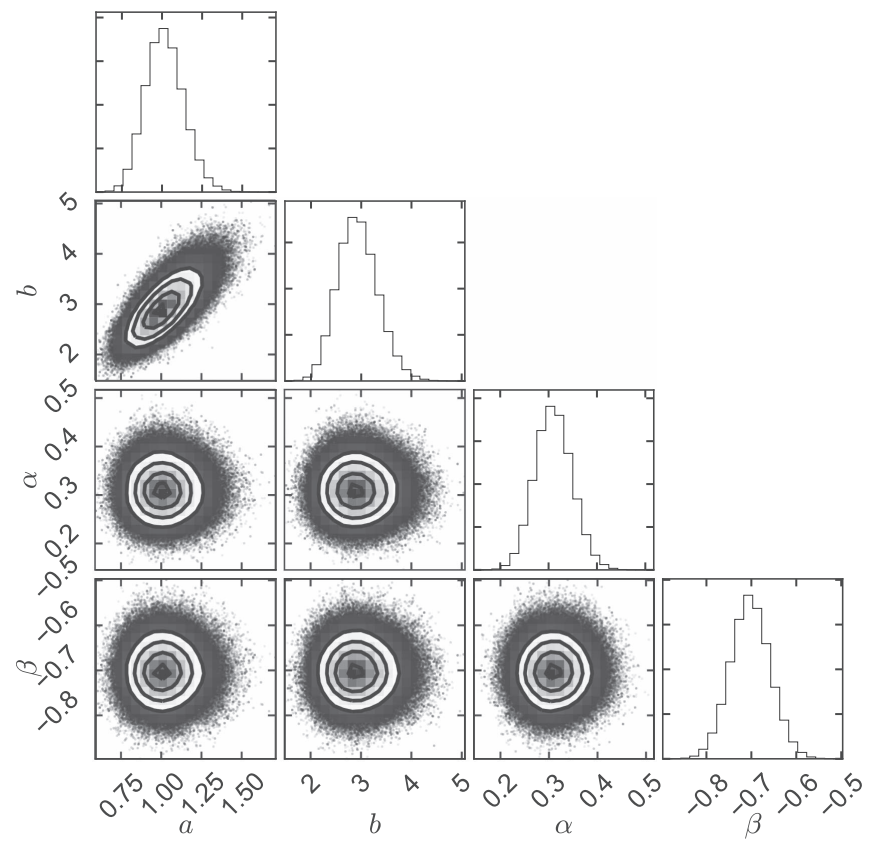

Figure 7. Two-dimensional posterior probability distributions on the four model parameters describing the 98 single systems and 17 multi-stellar systems as a single population of planets. The histograms represent the one-dimensional marginalized posterior probability distribution for each parameter.

two-population model $\mathcal{L}_{2}$ as

$$
\begin{aligned}
\mathcal{L}_{2}= & \sum_{i} \ln \left[\operatorname{prob}\left(e_{i}, m_{i}, P_{i} \mid M_{s}, \theta_{s}\right)\right] \\
& +\sum_{j} \ln \left[\operatorname{prob}\left(e_{j}, m_{j}, P_{j} \mid M_{m}, \theta_{m}\right)\right]+C,
\end{aligned}
$$

where the sum over $i$ includes all RV planet host systems with no companion star, the sum over $j$ includes all RV planet host systems with at least one companion star, and the model parameters have subscripts $s$ and $m$ to denote separate sets of parameters for single and multi-stellar systems, respectively. $C$ is the same constant from the one-population likelihood.

Using the Markov Chain Monte Carlo implemented by emcee python package's affine-invariant sampler (ForemanMackey et al. 2013), we compute the posterior probability distributions for the model parameters and determine the maximum likelihoods of each model, $\hat{\mathcal{L}}_{1}$ and $\hat{\mathcal{L}}_{2}$. Figures 7 and 8 show our calculated posteriors on each model parameter for each model. We determine whether a two-population model is justified by comparing the Bayesian Information Criteria (BIC) for these two models and by computing the Bayesian odds ratio. First, we compute the $\mathrm{BIC}$ as $\mathrm{BIC}=\ln (N) k-2 \hat{\mathcal{L}}$, where $N$ is the number of planets in the model fit and $k$ is the total number of parameters (i.e., four in the one-population model and eight in the two-population model). We find the difference between the two-population model BIC and the one-population model BIC to be 17, indicating that the two-population model is very strongly disfavored (Kass \& Raftery 1995). Second, we compute the Bayesian odds ratio as the probability of a one-population model divided by the probability of a two-population model, assuming both models have equal prior likelihoods and uniform priors on all model parameters, i.e.,

$$
\frac{\operatorname{prob}(1 \text { pop) }}{\operatorname{prob}(2 \operatorname{pop})}=\frac{\exp \hat{\mathcal{L}}_{1}}{\exp \hat{\mathcal{L}}_{2}} \frac{\prod_{x} 2 \pi \delta \theta_{x} / \Delta \theta_{x}}{\prod_{y} 2 \pi \delta \theta_{y} / \Delta \theta_{y}} .
$$

This is equal to the ratio of the evidence for each model in the case where the posteriors are n-dimensional normal distribution and when the priors are uniform. The first term on the right is the Bayes factor, and the second term is the Ockham factor to account for the model parameters. The product sum over $x$ covers the four parameters of the one-population model and the product sum over $y$ covers the eight parameters of the twomodel population. The $\Delta \theta$ term corresponds to a range in allowable parameter values from our uniform prior and $\delta \theta$ is the region in which the parameter yields a good fit. For this calculation, we calculate $\delta \theta$ as the $\theta$ interval, centered on the median value for $\theta$, that encompasses $68 \%$ of the posterior probability. Table 8 shows our assumed priors and fit errors used to calculate the odds ratio. We compute the Bayes factor to be 0.64 and the Ockham factor to be 135 , yielding an overall odds ratio that favors the one-population model over the twopopulation model 87 to 1 .

We also consider whether some of these companion stars could be more influential than others by repeating the above calculation with only the top nine (i.e., the top half) systems in Table 7. In this case, the BIC comparison still favors the onepopulation model with a $\Delta$ BIC of 13 . However, due to the smaller number of multi-stellar systems, the larger uncertainties on the model parameters for the multiple star component of the two-population model reduces the Ockham factor and yields an odds ratio of 2.6 to 1 , indicating no strong preference for either model. This also shows that our angular separation cutoff choice does not affect our results. Finally, we also repeat the above analysis including all planets in each system instead of only the innermost planet and find no difference in our results.

Based on these calculations, we conclude that there is no evidence for a difference in the eccentricity, mass, and orbital period distributions of the inner giant planet between single and multi-stellar systems within 6". Notably, in Bryan et al. (2016), we searched for outer planetary mass companions in these systems using long-term RV monitoring and found that the presence of such companions correlated with increased eccentricities for the inner planets in these systems, a difference significant at the $3 \sigma$ level.

\subsection{Constraining Additional Substellar Companions}

For the remaining single-star systems, we provide deep $\mathrm{K}$-band contrast curves from our imaging campaign. The average $5 \sigma K_{s}$ contrast at separations of $0.25,0.150$, and $1 . \prime 0$ are $4.96,6.64$, and 8.22 , respectively. These contrasts correspond to companions with masses of $0.16,0.09$, and 0.08 solar masses, respectively, around a Sun-like primary star. These upper limits on another object in a narrow field of view around these stars provide upper limits on the mass and semimajor axis of any potential additional substellar companions, which might be detected by other techniques, such as RV (e.g., Bryan et al. 2016) or astrometry. There is a strong theoretical motivation for continued RV monitoring of these systems, for instance, Petrovich \& Tremaine (2016) present a scenario for warm- and hot-Jupiter formation via planet-planet interactions and predicts additional giant planets at much wider 

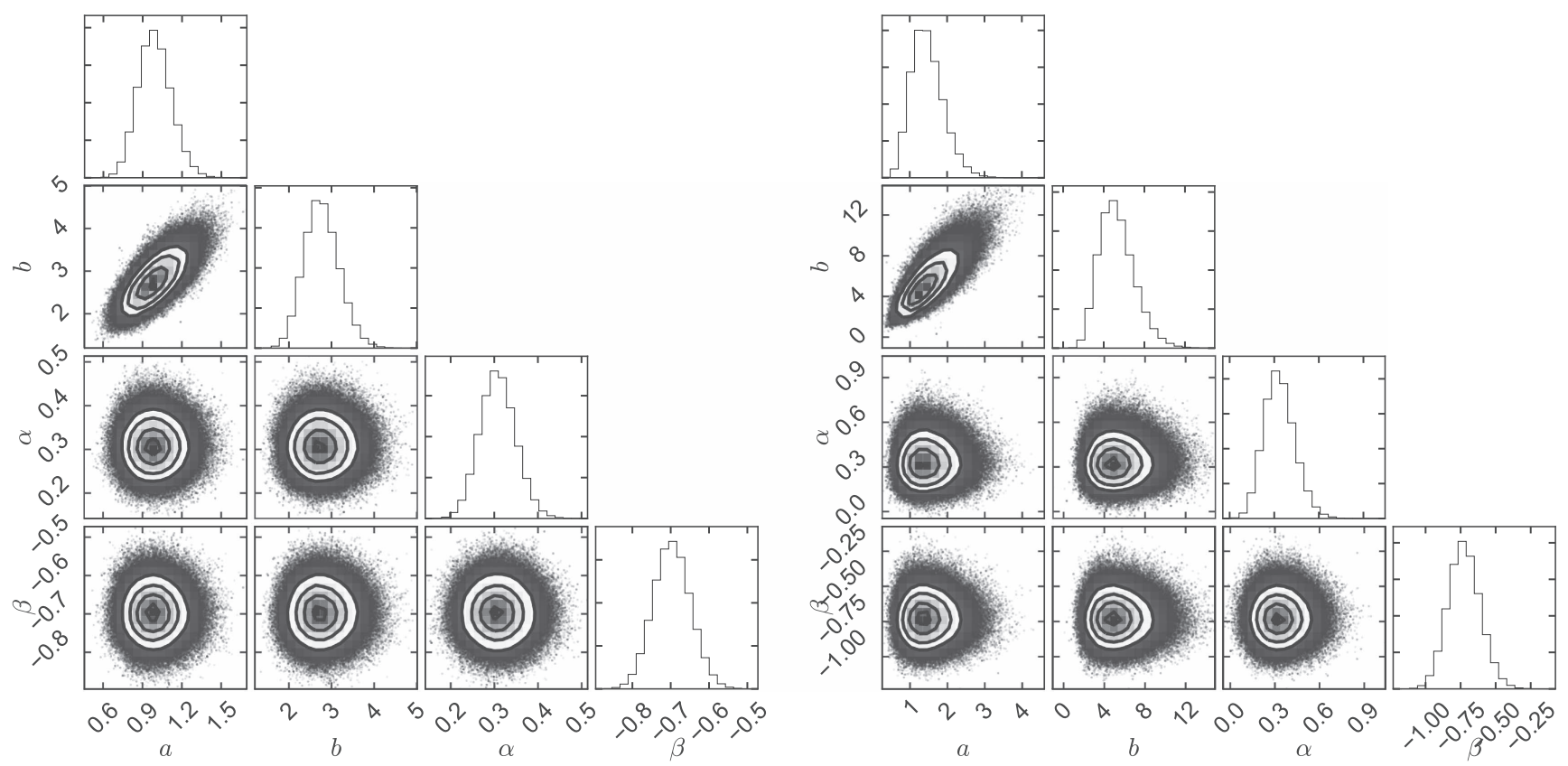

Figure 8. Posterior distributions on the eight model parameters describing the 98 single systems and 17 multi-stellar systems as two distinct populations of planets. The set of plots on the left correspond to the distribution of single systems and the set on the right corresponds to multi-stellar systems. The histograms represent the one-dimensional marginalized posterior probability distribution for each parameter.

separations that may be found via a long-term RV survey. A recent study (Hamers 2017) suggests that a companion star could interact with an additional planetary mass companion to induce the hot Jupiter to migrate via planetary Kozai-Lidov oscillations. Long-term RV surveys (e.g., Fischer et al. 2014b; Knutson et al. 2014; Montet et al. 2014; Bryan et al. 2016) currently have RV baselines of up to 25 years. Bryan et al. (2016) report 50\% completeness in their surveys for 1 Jupiter mass planets at $20 \mathrm{au}$ and for 10 Jupiter mass planets at $70 \mathrm{au}$. Hamers (2017) predict that Jupiter-sized planets at 40 au could cause migration. In the coming decades, RV surveys can find or rule out objects as small as a few Jupiter masses at separations of up to $40 \mathrm{au}$. These published contrast curves will help constrain the masses and semimajor axes of these potential future discoveries. In the future, Gaia astrometry will reach accuracies as low as $10 \mu$ as (microarcseconds) for stars with $V$ magnitudes of $7-12$ and $25 \mu$ as for stars with $V=15$ (Perryman et al. 2014). This would be accurate enough to determine the mutual inclination between widely separated giant planetary companions found in transiting and RV surveys (e.g., Buhler et al. 2016) and allow for constraints on planet-planet Kozai-Lidov migration.

\subsection{Astrometry of Triple Systems}

We study six hierarchical triple systems to determine the stellar orbital architectures. In these systems, the secondary and tertiary stars (the "inner binary" orbit) are close enough that we can detect orbit motion over our survey's baseline, unlike the orbits of our widely separated binary systems. Because of the hierarchical architecture, when we consider the "outer binary" orbit, the secondary and tertiary stars behave like a single body. We fit for all the orbital parameters of both the "inner" (B and $\mathrm{C}$ components) and "outer" orbits (A and BC components) using the Orbits For The Impatient method (OFTI), a Bayesian rejection sampling method described in Blunt et al. (2017). As
Table 8

Bayesian Model Comparison

\begin{tabular}{|c|c|c|c|}
\hline & \multirow{2}{*}{$\begin{array}{l}\text { One-population } \\
\text { Model }\end{array}$} & \multicolumn{2}{|c|}{ Two-population Model } \\
\hline & & $\begin{array}{c}\text { Single } \\
\text { Systems }\end{array}$ & $\begin{array}{l}\text { Multi-stellar } \\
\text { Systems }\end{array}$ \\
\hline \multicolumn{4}{|c|}{ Uniform prior ranges and $\Delta \theta$} \\
\hline$a$ & $(0,10), 10$ & $(0,10), 10$ & $(0,10), 10$ \\
\hline$b$ & $(0,10), 10$ & $(0,10), 10$ & $(0,15), 15$ \\
\hline$\alpha$ & $(-2,2), 4$ & $(-2,2), 4$ & $(-2,2), 4$ \\
\hline$\beta$ & $(-2,2), 4$ & $(-2,2), 4$ & $(-2,2), 4$ \\
\hline \multicolumn{4}{|c|}{ Fit results and uncertainties $\delta \theta$ for all single and multi-stellar systems } \\
\hline$\hat{\mathcal{L}}$ & -1331 & -1136 & -195 \\
\hline $\mathrm{N}$ parameters & 4 & 4 & 4 \\
\hline N systems & 115 & 98 & 17 \\
\hline$\delta a$ & 0.119 & 0.126 & 0.431 \\
\hline$\delta b$ & 0.397 & 0.409 & 1.745 \\
\hline$\delta \alpha$ & 0.038 & 0.041 & 0.104 \\
\hline$\delta \beta$ & 0.044 & 0.048 & 0.114 \\
\hline \multicolumn{4}{|c|}{$\begin{array}{l}\text { Fit results and uncertainties } \delta \theta \text { for all single and the top nine multi-stellar } \\
\text { systems }\end{array}$} \\
\hline$\hat{\mathcal{L}}$ & -1236 & -1136 & -97 \\
\hline $\mathrm{N}$ parameters & 4 & 4 & 4 \\
\hline N systems & 107 & 98 & 9 \\
\hline$\delta a$ & 0.125 & 0.125 & 0.852 \\
\hline$\delta b$ & 0.411 & 0.407 & 2.948 \\
\hline$\delta \alpha$ & 0.039 & 0.041 & 0.148 \\
\hline$\delta \beta$ & 0.047 & 0.048 & 0.224 \\
\hline
\end{tabular}

Note. Maximum likelihood, prior ranges, and fit uncertainties for a Bayesian model comparison of a one-population versus two-population model of giant planets discussed in Section 5.2.

demonstrated in de Rosa et al. (2015), Rameau et al. (2016), Bryan et al. (2016), and Blunt et al. (2017), OFTI calculates posterior distributions of orbital parameters that are identical to 
Table 9

Orbital Inclination Fits of Hierarchical Triple Systems

\begin{tabular}{|c|c|c|c|c|c|c|c|}
\hline \multirow{2}{*}{ System } & \multirow{2}{*}{$\begin{array}{l}\text { Planet Inclination } \\
\text { (deg) }\end{array}$} & \multicolumn{3}{|c|}{ Outer Orbit (ABC) Inclination (deg) } & \multicolumn{3}{|c|}{ Inner Orbit (BC) Inclination (deg) } \\
\hline & & $i_{\mathrm{P}, \max }$ & $68 \%$ C.I. & 95\% C.I. & $i_{\mathrm{P}, \max }$ & $68 \%$ C.I. & 95\% C.I. \\
\hline HD 43691 & unknown & 121.4 & $(131.9,163.4)$ & $(116.4,173.9)$ & 127.0 & $(131.0,162.8)$ & $(115.2,173.6)$ \\
\hline HD 142245 & unknown & 116.2 & $(107.3,139.7)$ & $(97.7,162.8)$ & 52.7 & $(21.8,53.9)$ & $(8.3,64.3)$ \\
\hline HD 207832 & unknown & 43.8 & $(18.5,53.9)$ & $(6.8,65.0)$ & 69.5 & $(54.2,75.7)$ & $(37.7,82.6)$ \\
\hline
\end{tabular}

Note. The most likely value $\left(i_{\mathrm{P}, \max }\right)$ and $68 \%$ and $95 \%$ confidence intervals (C.I.) for orbital inclinations of the triple star systems are computed from the orbital fits described in Section 5.4. HAT-P-8, HD 142245, and KELT-4A have the orientation of the binary pair misaligned with the entire triple system. HD 43691 and WASP12 are well aligned. HD 207832 is only marginally aligned (within the $68 \%$ confidence intervals). All of the transiting planet systems have binary companions on an orbital plane consistent with the planet's inclination at the $68 \%$ confidence interval (KELT-4A and WASP-12) or the 95\% confidence interval (HAT-P-8).

those produced by MCMC, but operates significantly faster when the input astrometry covers a short fraction of the total orbit $(<10 \%)$.

OFTI generates an initial orbit with a semimajor axis $a$ of $1 \mathrm{au}$, a position angle of nodes $\Omega$ of $0^{\circ}$, and other orbital parameters drawn from appropriate priors: uniform in eccentricity $e$, argument of periastron $\omega$, epoch of periastron passage $T_{0}$, and uniform in $\cos (i)$ (inclination angle). System mass and distance values are drawn from Gaussian distributions with medians and standard deviations equal to the measured values and observational uncertainties, and period $P$ is calculated from Kepler's third law. OFTI then scales $a$ and rotates $\Omega$ to match a single observational epoch, with observational errors included by adding random values drawn from Gaussian distributions with FWHM equal to the observed uncertainties in projected separation and position angle. Finally, the orbit's probability is computed from $p=e^{-\chi^{2} / 2}$. This value is compared with a uniform random number in $(0,1)$. If the chi-square probability is greater than this random number, the orbit is accepted. After many iterations of this process, probability distributions are calculated by computing histograms of the accepted sets of orbital parameters.

Table 9 describes the posterior distributions on the orbital inclinations of the inner (BC) and outer (ABC) orbits. One system, HD 142245, has secondary and tertiary stars with a mutual orbital plane that is misaligned with the plane of their orbits around the primary star. This misalignment is significant at the 95\% confidence level. Two systems, HD 43691 and WASP-12, have well-aligned orbital planes. Out of the transiting systems where the planet's inclination is measured, KELT-4A is the only system where companion stars are misaligned with the planet at the $95 \%$ confidence level. Note that RV fits do not provide the position angle of the nodes, so we are not sensitive to any misalignment perpendicular to our line of sight. Therefore, any offset in inclination angles in RV systems represents a minimum misalignment. Figure 9 shows the posterior distribution on the difference between the binary inclination and the planetary inclination for the three triple star systems with transiting planets. We find that both the inner and outer binaries of these systems are no more or less likely to be aligned or misaligned with the transiting planet. The transiting planets have inclinations close to edge-on, so this implies that the outer and inner orbits favor neither an edge-on nor a faceon orbit. Although there are only three systems in our sample, it would be interesting to investigate the general distribution of $i_{\mathrm{ABC}}-i_{b}$ and $i_{\mathrm{BC}}-i_{b}$ with more transiting systems. Although we only discuss the inclination probability distribution here, we plot the probability contours for all seven orbital parameters for one sample system in Figure 10, we summarize the posteriors on all orbital elements for all triple systems in Tables 11-16, and we provide posterior samples of all parameters for all six triple systems online.

\subsection{Summary of Current Observational Constraints on the Effects of Companions on Giant Planet Formation and Migration}

There is a considerable body of literature focused on possible formation and migration mechanisms for hot and warm Jupiters. In this section, we review this literature and the current work to constrain these theories via surveys for planetary and stellar companions to hot and warm Jupiters. We also put the results of this work in context with these other surveys. Although there exist variations on the strict definitions of a "hot" versus a "warm" Jupiter, for brevity in this discussion, we refer to giant planets with masses greater than $0.1 M_{\text {Jup }}$ and semimajor axes less than 0.1 au as "hot" Jupiters and planets with semimajor axes between 0.1 and 1.0 au as "warm" Jupiters.

Possible migration mechanisms include both interactions with the protoplanetary gas disk and with other planetary or stellar companions in the system. Formation followed by gas disk migration must occur quickly because the gas disk only survives for 1-10 million years (Pollack et al. 1996; Haisch et al. 2001; Hernández et al. 2009). This formation channel is expected to create hot Jupiters on low eccentricity orbits (e.g., Goldreich \& Tremaine 1980; Lin \& Papaloizou 1986; Tanaka et al. 2002). On the other hand, interactions such as gravitational scattering with other planets (e.g., Chatterjee et al. 2008; Wu \& Lithwick 2011; Beaugé \& Nesvorný 2012; Lithwick \& Wu 2014; Petrovich 2015) or stars via stellar Kozai-Lidov oscillations (e.g., Wu \& Murray 2003; Fabrycky \& Tremaine 2007; Naoz et al. 2012, 2013; Storch et al. 2014) in the system could create hot Jupiters on more eccentric orbits.

Recent studies have used characteristics of the existing giant exoplanet population to attempt to distinguish between migration mechanisms. Dawson et al. (2015) points out a lack 

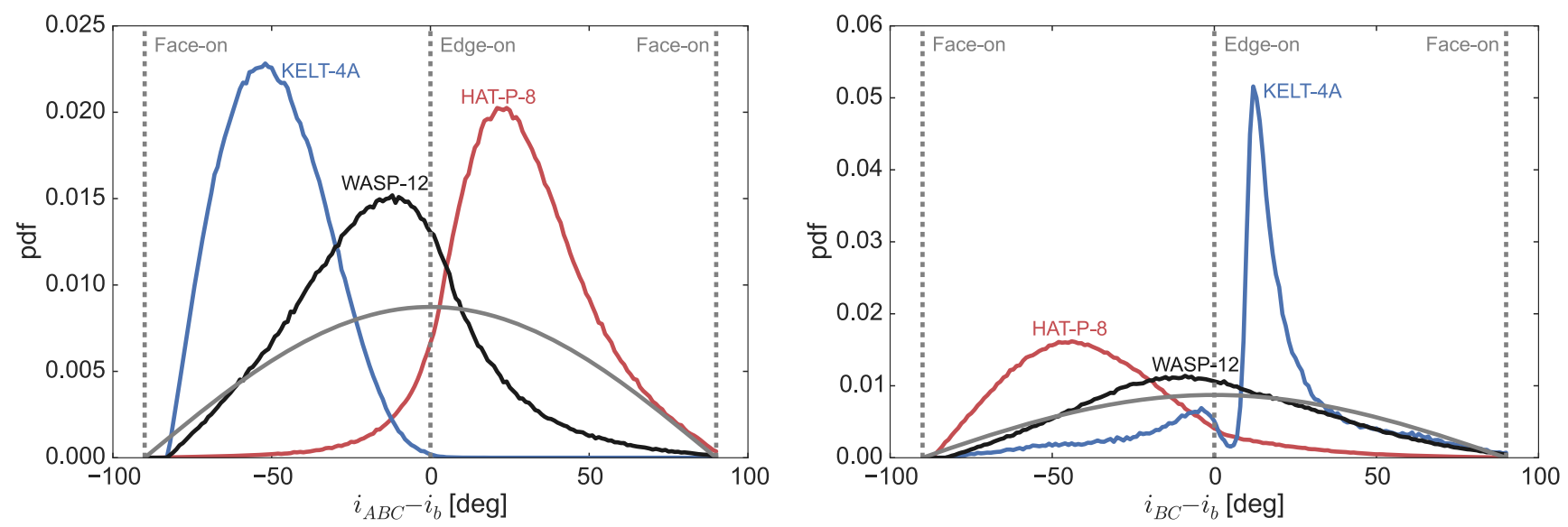

Figure 9. Left: posterior distributions on the difference between the outer binary's inclination and the transiting planet's inclination. Posteriors on the outer binary's inclination are computed from OFTI, while the planet's inclination comes from Latham et al. (2009), Eastman et al. (2016), and Collins et al. (2017) for HAT-P-8b, KELT-4Ab, and WASP-12b, respectively. The solid gray pdf represents the prior on $\Delta i$ for an edge-on planet. Right: the same for the difference between the inner binary's inclination and the transiting planet's inclination.

Table 10

Contrast Curves for All Targets

\begin{tabular}{|c|c|c|c|c|c|}
\hline Star & Bandpass & Date & $\begin{array}{c}\text { Separation } \\
(\operatorname{arcsec})\end{array}$ & $\begin{array}{l}5 \sigma \text { contrast } \\
\text { (magnitudes) }\end{array}$ & $\begin{array}{l}\text { Directional } \\
\text { completeness }\end{array}$ \\
\hline \multirow[t]{2}{*}{ GJ 317} & $\mathrm{Kc}$ & $\begin{array}{c}2014 \\
\text { Nov } \\
07\end{array}$ & 0.154 & 2.87 & 100.0 \\
\hline & & & $\begin{array}{c}0.252 \\
0.350 \\
0.449 \\
\ldots\end{array}$ & $\begin{array}{c}4.52 \\
5.53 \\
6.33 \\
\ldots\end{array}$ & $\begin{array}{c}100.0 \\
100.0 \\
100.0 \\
\ldots\end{array}$ \\
\hline \multirow[t]{3}{*}{ GJ-433 } & $\mathrm{Kc}$ & $\begin{array}{c}2015 \\
\text { Jan } \\
09\end{array}$ & 0.154 & 3.07 & 100.0 \\
\hline & & & $\begin{array}{l}0.251 \\
0.350 \\
0.449\end{array}$ & $\begin{array}{l}4.90 \\
5.77 \\
6.52\end{array}$ & $\begin{array}{l}100.0 \\
100.0 \\
100.0\end{array}$ \\
\hline & & & $\cdots$ & $\ldots$ & $\cdots$ \\
\hline
\end{tabular}

Note. Best $5 \sigma$ contrast curve for each target in the indicated bandpass. The full table with all 144 targets will be available in the comma separated value (CSV) format.

(This table is available in its entirety in machine-readable form.)

of high-eccentricity warm Jupiters in the Kepler sample, suggesting that multi-body processes are unlikely to form hot Jupiters. However, Petrovich et al. (2014) showed that planetplanet scattering at separations within 0.2 au would not excite high eccentricities. In this scenario, the giant planets could scatter off each other without creating high-eccentricity warm Jupiters. Stellar Kozai-Lidov migration is expected to create misaligned hot Jupiters, but our recent stellar companion surveys (Ngo et al. 2015; Piskorz et al. 2015) find no correlation between the incidence of hot Jupiter misalignment and stellar multiplicity. In addition, we place an upper limit of $20 \%$ for systems experiencing Kozai-Lidov migration (Ngo et al. 2016). Furthermore, Schlaufman \& Winn (2016) argue that a planet-planet scattering scenario for hot-Jupiter migration would predict that hot Jupiters would have fewer giant planet companions interior to the water-ice line as compared to warm Jupiters. They examined RV-detected hot- and warmJupiter systems and found that hot Jupiters are just as likely to host exterior giant planet companions as warm Jupiters. In addition, short-period giant planets found around young $\mathrm{T}$ Tauri stars, such as CI Tau b (Johns-Krull et al. 2016), have lifetimes too short for migration via multi-body interactions. These results disfavor high-eccentricity hot-Jupiter migration and would instead suggest that disk migration or in situ formation scenarios are more likely for short-period giant planets.

$\mathrm{RV}$ monitoring surveys have found that long-period giant planet companions to transiting hot Jupiters (Knutson et al. 2014) and RV-detected giant planets (Bryan et al. 2016) are common. In Bryan et al. (2016), we find that $52 \% \pm 5 \%$ of the RV giant planet systems host additional long-period planetary mass companions (5-20 au, 1-20 Jupiter masses). In addition, the gas giant planets beyond 0.1 au have, on average, higher orbital eccentricities when they have an outer companion. This finding is consistent with work by Petrovich \& Tremaine (2016), showing that secular planet-planet interactions can account for most of the observed hot-Jupiter population; however, these interactions fail to reproduce the known warm-Jupiter planets. These types of interactions can also excite large mutual inclinations, resulting in misaligned planetary systems (e.g., see Johansen et al. 2012; Morton \& Winn 2014; Ballard \& Johnson 2016; Becker \& Adams 2016; Spalding \& Batygin 2016). Finally, these additional planets can also interact with the inner giant planets through planet-planet Kozai-Lidov effects (Dawson \& Chiang 2014).

The presence of massive planetary and/or stellar companions in these systems can also have important implications for in situ formation models. Some in situ models (e.g., Boley et al. 2016) invoke a globally enhanced disk mass or a local concentration of solids in the region of interest, both of which would affect the locations and masses of other gas giant planets formed in the same disk. Alternatively, other in situ models (e.g., Batygin et al. 2016) form hot Jupiters from rapid gas accretion onto super-Earth planets, which are already commonly found at short periods (Fressin et al. 2013). Batygin et al. (2016) also predict that hot Jupiters that formed in situ should also have additional low-mass planets with orbital periods of less than 100 days. RV surveys of known planetary systems find preliminary evidence that that hot Jupiters are 


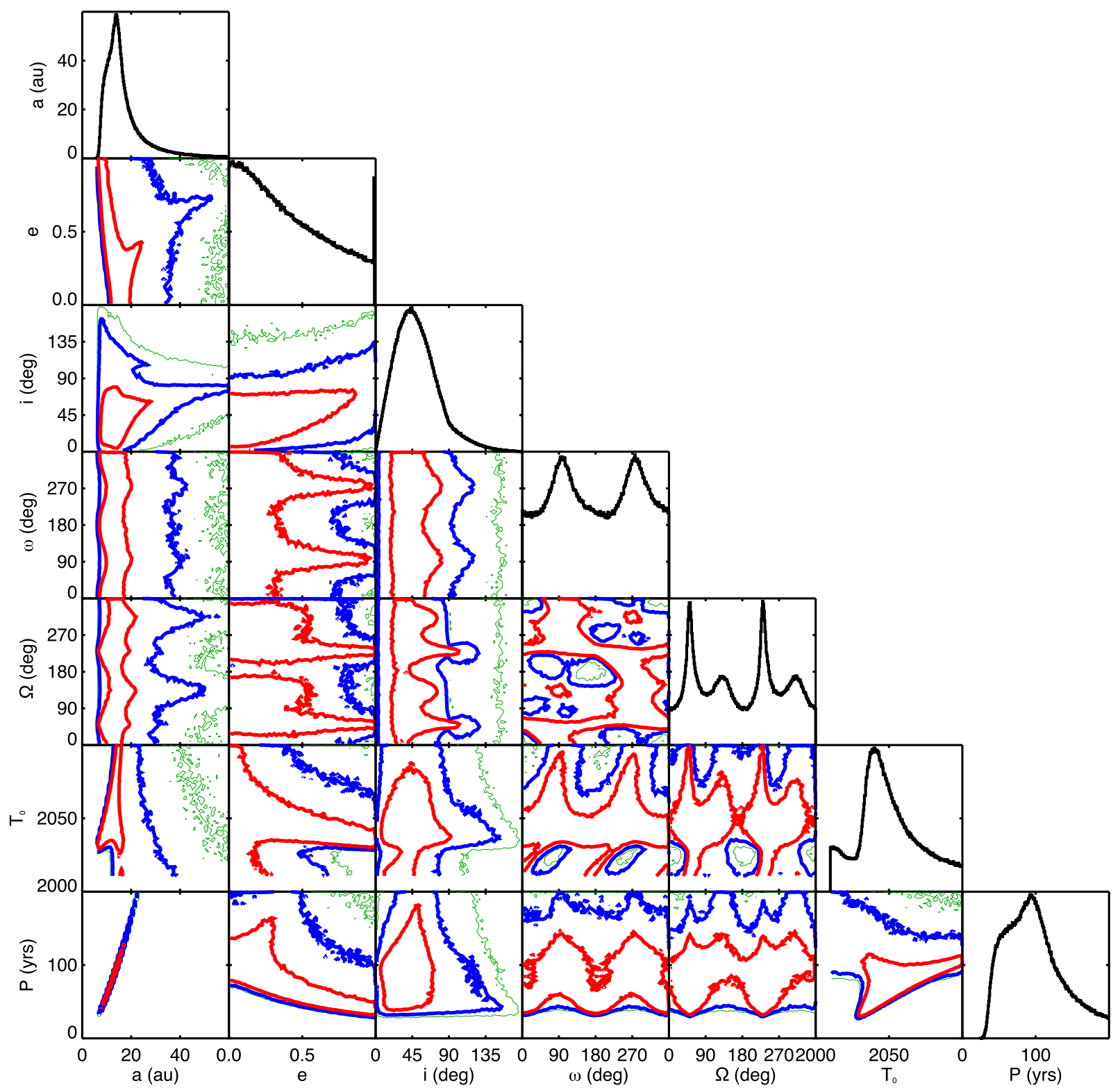

Figure 10. Two-dimensional probability contours for each pair of the seven OFTI-fitted orbital parameters. The red, blue, and green contours represent regions containing $68 \%, 95 \%$, and $99.7 \%$ of the marginalized probabilities. The black histograms show the one-dimensional marginalized probability distributions for each orbital parameter. This representative set of panels is for the inner orbit of the system with the best constraints, HAT-P-8. Tables 11 through 16 summarize the fit results for this system and all triple systems.

more likely to host an additional companion than warm Jupiters (Bryan et al. 2016). Many theoretical studies of planet formation in binary star systems predict that the presence of a second star would be detrimental for planet formation by exciting or removing planetesimals in the protoplanetary disk (e.g., Mayer et al. 2005; Pichardo et al. 2005). Stellar companions could also eject planets after formation (e.g., Kaib et al. 2013; Zuckerman 2014). For close (less than 50 au separation) binaries, the current observational evidence appears to support this view. Kraus et al. (2012) found that two-thirds of young stars with stellar companions within 40 au lose their protoplanetary disks within 1 million years, while systems with more distant companions have disk lifetimes that are comparable to single-star systems. In a follow-up study, Kraus et al. (2016) surveyed 386 Kepler planet host stars and showed that these stars are three times less likely to have a stellar companion within 50 au than non-planet hosting field stars. Wang et al. (2014) also came to a similar conclusion in their survey of 56 Kepler planet host stars.

Although current studies indicate that planet formation is suppressed in close stellar binaries, there are many examples of known planet-hosting stars in relatively wide (greater than $50 \mathrm{au})$ binaries. The two most recent directly imaged giant planet systems, 51 Eri b (Macintosh et al. 2015; Montet et al. 2015) and HD $131399 \mathrm{Ab}$ (Wagner et al. 2016), are both part of hierarchical triple systems. Ngo et al. (2016) surveyed a sample of 77 transiting hot-Jupiter host stars and found that $47 \% \pm 7 \%$ of these systems have a directly imaged stellar companion. Other near-infrared diffraction-limited direct imaging surveys for stellar companions to transiting close-in 
giant planet systems have found companion fractions consistent with our result (Adams et al. 2013; Wang et al. 2015b; Wöllert \& Brandner 2015; Wöllert et al. 2015; Evans et al. 2016). In Ngo et al. (2016), we found that hot-Jupiter host stars have fewer close-in stellar companions (projected separations of less than $50 \mathrm{au}$ ) than field stars; however, they are three times more likely to have a wide companion star (projected separations greater than $50 \mathrm{au}$ ) than field stars. These companions may play some role in enhancing planet formation.

In this work, we considered the effects of stellar companions on gas giant planets at intermediate $(0.1-5 \mathrm{au})$ separations. We conducted a large survey for stellar companions to RV-detected warm and cool $(a<5$ au) Jupiters. We show that there is currently no evidence for a correlation between the incidence of a stellar companion and the gas giant planet's mass, orbital eccentricity, or orbital period. This suggests that the presence or absence of a stellar companion do not significantly alter the formation or orbital evolution of gas giant planets at intermediate separations. Given the mass ratios and projected separations of the stellar companions in our sample, it seems unlikely that these companions could have induced KozaiLidov oscillations in most of the systems observed. This result is consistent with the absence of increased planet eccentricities in multi-stellar systems, and lends more weight to in situ or planet-planet scattering theories for the formation of warm Jupiters. Our results also increase the number of known RVplanet systems with companion stars; these systems can serve as case studies for models of planet formation and migration in multiple star systems.

\section{Summary}

We carry out an AO imaging search for stellar companions around 144 stars with RV-detected giant planets. The sample is the largest survey for stellar companions around RV planet hosts to date and includes 123 stars from our previous longterm RV monitoring study (Bryan et al. 2016). We detect 11 comoving multi-stellar systems, corresponding to a raw companion fraction of $7.6 \% \pm 2.3 \%$. This value is consistent with other surveys for stellar companions around RV planet systems, but is much lower than the stellar companion fraction for transiting gas giant planets because of strong biases against multi-stellar systems in sample selection for RV surveys.

Three of the multi-stellar systems are presented for the first time in this work (HD 30856, HD 86081, and HD 207832). We confirm common proper motion for another three systems (HD 43691, HD 116029, and HD 164509). These six new confirmed multi-stellar RV systems increase the total number of systems with known companions closer than $66^{\prime \prime}$ to 22 . We compare the mass, orbital eccentricity, and semimajor axis distribution of the innermost planet in the multi-stellar systems with those of the innermost planet in the single-star systems. Our analysis indicates that these distributions are the same for both single and multi-stellar systems. This suggests that the observed stellar companions do not significantly alter the properties of the giant planet in these systems. Even when limiting our comparison to the most dynamically influential (i.e., the most massive and closest in) stellar companions, we find no evidence for any difference in the distribution of planet orbital properties. These results appear to disfavor Kozai-type migration processes, and are consistent with both in situ formation and planet-planet scattering.
We also compute contrast curves for all 144 surveyed targets. These provide upper limits on the remaining undetected stellar and substellar companions, and can be used to constrain the masses of any additional companions found in long-term $\mathrm{RV}$ monitoring surveys. We note that there is great value in continued RV monitoring of these systems because the presence or absence of more distant (>5-10 au) planetary mass companions would provide invaluable insights into the likely formation and migration histories of these systems. Another potentially valuable study would be to obtain AO imaging data for a control sample of stars from the CPS survey, which are not currently known to host planets. As in Eggenberger et al. (2007), this sample would allow us to empirically measure the selection biases against multi-stellar systems in our current planet-hosting star sample and calculate a stellar multiplicity rate for that sample that can be directly compared to that of field stars.

Finally, in our survey's hierarchical triple systems (HD 43691, HD 142245, and HD 207832), the secondary and tertiary stellar components are on very tight orbits (less than $10 \mathrm{au}$ ), so it is possible to measure orbital motion over the several year baseline of our survey. We fit orbital parameters for all three stars in these three triple systems as well as three additional triple systems from transiting planet surveys. We show that these orbital fits allow us to constrain the geometry of the triple system (e.g., edge-on or face-on), which has implications for the dynamical evolution of the planet orbits in these systems.

We would like to thank John A Johnson, Christopher Spalding, and Benjamin Montet for helpful discussions. We also appreciate the useful suggestions from the anonymous referee and the statistics editor. This work was supported by NASA grant NNX14AD24G. H.N. is grateful for funding support from the Natural Sciences and Engineering Research Council of Canada and the NASA Earth and Space Science Fellowship Program grant NNX15AR12H. H.A.K. acknowledges support from the Sloan Foundation. E.L.N. and S.C.B. are supported by NASA grant NNX14AJ80G.

This work was based on observations at the W. M. Keck Observatory granted by the California Institute of Technology. We thank the observers who contributed to the measurements reported here and acknowledge the efforts of the Keck Observatory staff. We extend special thanks to those of Hawaiian ancestry on whose sacred mountain of Mauna Kea we are privileged to be guests.

Facility: Keck:II.

Software: Astropy (Astropy Collaboration et al. 2013), OFTI (Blunt et al. 2017), emcee (Foreman-Mackey et al. 2013), corner.py (Foreman-Mackey 2016).

\section{Appendix OFTI Orbit Fits}

The full orbit fit results from Section 5.4 are described in this appendix. Figure 10 displays the two-dimensional probability distribution for the orbital parameters of one typical system. Tables 11 through 16 summarize the fit results for each system. Samples of the posteriors for all fit parameters for all systems are available online as FITS tables. 
Table 11

OFTI Fit Summary for Triple System HAT-P-8

\begin{tabular}{|c|c|c|c|c|c|c|}
\hline$x$ & Median & $x \chi^{2}, \min$ & $x_{\mathrm{P}, \max }$ & $68 \%$ C.I. & $95 \%$ C.I. & 99.7\% C.I. \\
\hline \multicolumn{7}{|c|}{ Outer orbit $\left(\min \chi^{2}=25.701, \chi^{2}\right.$ at highest probability $\left.=25.854\right)$} \\
\hline$a(\mathrm{au})$ & 241.9 & 178.7 & 111.5 & $(160.2,491.4)$ & $(128.3,1713.3)$ & $(112.9,8324.6)$ \\
\hline$e$ & 0.582 & 0.413 & 0.832 & $(0.210,0.868)$ & $(0.031,0.980)$ & $(0.002,0.990)$ \\
\hline$i(\mathrm{deg})$ & 113.4 & 115.3 & 118.8 & $(94.8,136.3)$ & $(69.6,161.2)$ & $(25.6,175.2)$ \\
\hline$\omega(\operatorname{deg})$ & 91.3 & 162.6 & 22.4 & $(36.4,144.3)$ & $(5.5,174.6)$ & $(0.3,179.7)$ \\
\hline$\Omega(\operatorname{deg})$ & 128.5 & 134.4 & 327.8 & $(50.6,148.8)$ & $(6.8,173.7)$ & $(0.4,179.6)$ \\
\hline$T_{0}$ (years) & 3312 & 2892 & 2556 & $(2687,6377)$ & $(2393,30869)$ & $(2035,325732)$ \\
\hline$P$ (years) & 3091 & 1916 & 942 & $(1665,8956)$ & $(1191,58222)$ & $(978,625040)$ \\
\hline \multicolumn{7}{|c|}{ Inner orbit $\left(\min \chi^{2}=0.320, \chi^{2}\right.$ at highest probability $\left.=0.787\right)$} \\
\hline$a(\mathrm{au})$ & 14.9 & 15.8 & 9.3 & $(10.5,25.3)$ & $(7.9,73.4)$ & $(6.8,366.7)$ \\
\hline$e$ & 0.347 & 0.193 & 0.457 & $(0.098,0.728)$ & $(0.014,0.955)$ & $(0.001,0.990)$ \\
\hline$i(\mathrm{deg})$ & 47.7 & 3.5 & 44.7 & $(24.9,75.6)$ & $(9.4,118.6)$ & $(2.3,160.5)$ \\
\hline$\omega(\mathrm{deg})$ & 94.2 & 242.9 & 288.4 & $(34.9,147.6)$ & $(4.9,175.1)$ & $(0.3,179.7)$ \\
\hline$\Omega(\operatorname{deg})$ & 80.3 & 326.6 & 93.4 & $(39.2,143.0)$ & $(6.7,173.4)$ & $(0.4,179.6)$ \\
\hline$T_{0}$ (years) & 2056 & 2010 & 2034 & $(2035,2135)$ & $(2014,2603)$ & $(2010,8439)$ \\
\hline$P$ (years) & 105 & 116 & 52 & $(63,234)$ & $(41,1159)$ & $(32,12930)$ \\
\hline
\end{tabular}

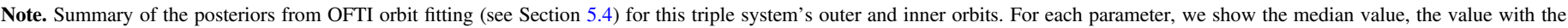
lowest $\chi^{2}\left(x_{\chi^{2}, \min }\right)$, the most likely value $\left(x_{\mathrm{P}, \max }\right)$, and three confidence intervals (C.I.).

Table 12

OFTI Fit Summary for Triple System HD 43691

\begin{tabular}{|c|c|c|c|c|c|c|}
\hline$x$ & Median & $x_{\chi^{2}, \min }$ & $x_{P, \max }$ & $68 \%$ C.I. & 95\% C.I. & $99.7 \%$ C.I. \\
\hline \multicolumn{7}{|c|}{ Outer orbit $\left(\min \chi^{2}=7.089, \chi^{2}\right.$ at highest probability $\left.=7.444\right)$} \\
\hline$a(\mathrm{au})$ & 452.1 & 401.5 & 162.2 & $(341.5,709.3)$ & $(254.5,1547.9)$ & $(190.4,4882.8)$ \\
\hline$e$ & 0.229 & 0.169 & 0.312 & $(0.068,0.474)$ & $(0.010,0.730)$ & $(0.001,0.909)$ \\
\hline$i(\mathrm{deg})$ & 148.3 & 165.0 & 121.4 & $(131.9,163.4)$ & $(116.4,173.9)$ & $(103.1,178.5)$ \\
\hline$\omega(\mathrm{deg})$ & 92.0 & 143.5 & 223.4 & $(28.4,152.4)$ & $(4.0,176.0)$ & $(0.2,179.8)$ \\
\hline$\Omega(\mathrm{deg})$ & 98.3 & 210.6 & 49.3 & $(28.9,153.5)$ & $(4.1,175.9)$ & $(0.2,179.7)$ \\
\hline$T_{0}$ (years) & 5071 & 8249 & 3059 & $(2930,11372)$ & $(2130,34638)$ & $(2017,187612)$ \\
\hline$P$ (years) & 7518 & 6569 & 1649 & $(4935,14775)$ & $(3172,47655)$ & $(2050,266357)$ \\
\hline \multicolumn{7}{|c|}{ Inner orbit $\left(\min \chi^{2}=2.256, \chi^{2}\right.$ at highest probability $\left.=2.974\right)$} \\
\hline$a(\mathrm{au})$ & 11.2 & 16.6 & 7.2 & $(8.5,17.9)$ & $(6.5,39.8)$ & $(5.0,127.7)$ \\
\hline$e$ & 0.214 & 0.333 & 0.310 & $(0.064,0.456)$ & $(0.009,0.727)$ & $(0.001,0.910)$ \\
\hline$i(\mathrm{deg})$ & 147.4 & 119.8 & 127.0 & $(131.0,162.8)$ & $(115.2,173.6)$ & $(102.3,178.5)$ \\
\hline$\omega(\operatorname{deg})$ & 79.1 & 148.6 & 256.2 & $(25.6,148.7)$ & $(3.9,176.0)$ & $(0.2,179.8)$ \\
\hline$\Omega(\operatorname{deg})$ & 79.7 & 278.9 & 272.8 & $(33.1,128.8)$ & $(5.9,173.4)$ & $(0.4,179.6)$ \\
\hline$T_{0}$ (years) & 2029 & 2027 & 2032 & $(2018,2056)$ & $(2012,2172)$ & $(2010,2952)$ \\
\hline$P$ (years) & 74 & 138 & 39 & $(49,151)$ & $(33,499)$ & $(22,2863)$ \\
\hline
\end{tabular}

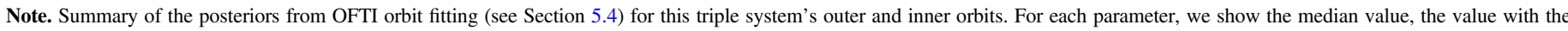
lowest $\chi^{2}\left(x_{\chi^{2}, \min }\right)$, the most likely value $\left(x_{\mathrm{P}, \max }\right)$, and three confidence intervals (C.I.).

Table 13

OFTI Fit Summary for Triple System HD 142245

\begin{tabular}{|c|c|c|c|c|c|c|}
\hline$x$ & Median & $x_{\chi^{2}, \text { min }}$ & $x_{\mathrm{P}, \max }$ & $68 \%$ C.I. & $95 \%$ C.I. & $99.7 \%$ C.I. \\
\hline \multicolumn{7}{|c|}{ Outer orbit $\left(\min \chi^{2}=21.154, \chi^{2}\right.$ at highest probability $\left.=21.337\right)$} \\
\hline$a(\mathrm{au})$ & 273.2 & 309.5 & 111.9 & $(182.0,556.9)$ & $(140.6,1694.7)$ & $(115.1,6011.4)$ \\
\hline$e$ & 0.543 & 0.568 & 0.711 & $(0.211,0.785)$ & $(0.032,0.918)$ & $(0.002,0.982)$ \\
\hline$i(\operatorname{deg})$ & 120.4 & 118.3 & 116.2 & $(107.3,139.7)$ & $(97.7,162.8)$ & $(91.6,175.7)$ \\
\hline$\omega(\operatorname{deg})$ & 85.8 & 268.6 & 161.2 & $(38.4,139.8)$ & $(6.0,173.9)$ & $(0.4,179.6)$ \\
\hline$\Omega(\operatorname{deg})$ & 91.3 & 207.6 & 163.9 & $(24.2,168.6)$ & $(2.5,177.8)$ & $(0.1,179.9)$ \\
\hline$T_{0}$ (years) & 3060 & 4428 & 2312 & $(2573,5746)$ & $(2343,23231)$ & $(2034,167483)$ \\
\hline$P$ (years) & 2747 & 3351 & 712 & $(1493,8005)$ & $(1012,42425)$ & $(749,285552)$ \\
\hline \multicolumn{7}{|c|}{ Inner orbit $\left(\min \chi^{2}=6.811, \chi^{2}\right.$ at highest probability $\left.=8.294\right)$} \\
\hline$a(\mathrm{au})$ & 6.4 & 44.1 & 5.1 & $(5.1,8.7)$ & $(4.4,15.1)$ & $(3.9,43.0)$ \\
\hline$e$ & 0.440 & 0.924 & 0.705 & $(0.210,0.619)$ & $(0.033,0.774)$ & $(0.002,0.897)$ \\
\hline$i(\mathrm{deg})$ & 39.5 & 64.5 & 52.7 & $(21.8,53.9)$ & $(8.3,64.3)$ & $(1.9,74.6)$ \\
\hline$\omega(\mathrm{deg})$ & 97.2 & 133.8 & 90.1 & $(38.9,145.9)$ & $(5.5,174.5)$ & $(0.4,179.6)$ \\
\hline$\Omega(\mathrm{deg})$ & 75.5 & 33.4 & 15.0 & $(37.3,115.6)$ & $(7.7,170.7)$ & $(0.5,179.5)$ \\
\hline$T_{0}$ (years) & 2012 & 2013 & 2012 & $(2011,2021)$ & $(2010,2030)$ & $(2010,2060)$ \\
\hline$P$ (years) & 16 & 289 & 11 & $(11,25)$ & $(9,58)$ & $(8,279)$ \\
\hline
\end{tabular}

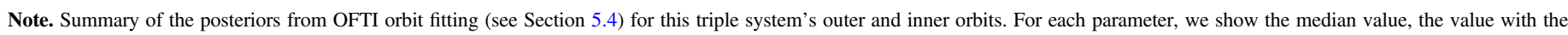
lowest $\chi^{2}\left(x_{\chi^{2}, \min }\right)$, the most likely value ( $\left.x_{\mathrm{P}, \max }\right)$, and three confidence intervals (C.I.). 
Table 14

OFTI Fit Summary for Triple System HD 207832

\begin{tabular}{|c|c|c|c|c|c|c|}
\hline$x$ & Median & $x_{\chi^{2}, \min }$ & $x_{\mathrm{P}, \max }$ & $68 \%$ C.I. & $95 \%$ C.I. & $99.7 \%$ C.I. \\
\hline \multicolumn{7}{|c|}{ Outer orbit $\left(\min \chi^{2}=3.760, \chi^{2}\right.$ at highest probability $\left.=4.301\right)$} \\
\hline$a(\mathrm{au})$ & 171.5 & 158.0 & 60.8 & $(116.4,299.0)$ & $(86.9,684.2)$ & $(68.8,2131.0)$ \\
\hline$e$ & 0.713 & 0.701 & 0.680 & $(0.567,0.809)$ & $(0.359,0.900)$ & $(0.122,0.966)$ \\
\hline$i(\mathrm{deg})$ & 36.2 & 30.6 & 43.8 & $(18.5,53.9)$ & $(6.8,65.0)$ & $(1.6,72.1)$ \\
\hline$\omega(\operatorname{deg})$ & 64.0 & 232.3 & 20.4 & $(23.1,133.7)$ & $(4.0,175.8)$ & $(0.3,179.8)$ \\
\hline$\Omega(\operatorname{deg})$ & 67.9 & 229.8 & 42.1 & $(41.3,104.3)$ & $(9.9,168.0)$ & $(0.7,179.4)$ \\
\hline$T_{0}$ (years) & 3925 & 3765 & 2357 & $(3007,6644)$ & $(2604,18356)$ & $(2057,92862)$ \\
\hline$P$ (years) & 2083 & 1900 & 456 & $(1159,4821)$ & $(743,16659)$ & $(522,91976)$ \\
\hline \multicolumn{7}{|c|}{ Inner orbit $\left(\min \chi^{2}=7.067, \chi^{2}\right.$ at highest probability $\left.=7.376\right)$} \\
\hline$a(\mathrm{au})$ & 6.7 & 21.9 & 5.0 & $(4.5,12.1)$ & $(3.4,26.3)$ & $(2.8,81.1)$ \\
\hline$e$ & 0.305 & 0.538 & 0.597 & $(0.093,0.613)$ & $(0.013,0.847)$ & $(0.001,0.958)$ \\
\hline$i(\mathrm{deg})$ & 65.9 & 75.5 & 69.5 & $(54.2,75.7)$ & $(37.7,82.6)$ & $(15.2,87.5)$ \\
\hline$\omega(\mathrm{deg})$ & 61.2 & 204.7 & 63.1 & $(28.1,141.0)$ & $(4.8,175.1)$ & $(0.3,179.7)$ \\
\hline$\Omega(\operatorname{deg})$ & 105.2 & 285.3 & 275.9 & $(89.9,120.8)$ & $(50.6,135.9)$ & $(7.8,170.0)$ \\
\hline$T_{0}$ (years) & 2026 & 2236 & 2024 & $(2019,2051)$ & $(2011,2160)$ & $(2010,2852)$ \\
\hline$P$ (years) & 39 & 231 & 25 & $(22,95)$ & $(14,302)$ & $(10,1639)$ \\
\hline
\end{tabular}

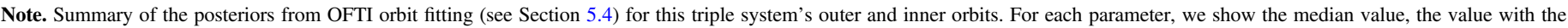
lowest $\chi^{2}\left(x_{\chi^{2}, \min }\right)$, the most likely value $\left(x_{\mathrm{P}, \max }\right)$, and three confidence intervals (C.I.).

Table 15

OFTI Fit Summary for Triple System KELT-4A

\begin{tabular}{|c|c|c|c|c|c|c|}
\hline$x$ & Median & $x \chi^{2}$, min & $x_{\mathrm{P}, \max }$ & $68 \%$ C.I. & $95 \%$ C.I. & 99.7\% C.I. \\
\hline \multicolumn{7}{|c|}{ Outer orbit $\left(\min \chi^{2}=0.039, \chi^{2}\right.$ at highest probability $\left.=0.900\right)$} \\
\hline$a(\mathrm{au})$ & 425.1 & 478.9 & 369.1 & $(312.4,725.5)$ & $(239.2,1657.8)$ & $(193.5,5227.0)$ \\
\hline$e$ & 0.406 & 0.561 & 0.229 & $(0.145,0.660)$ & $(0.021,0.846)$ & $(0.001,0.951)$ \\
\hline$i(\mathrm{deg})$ & 32.7 & 5.8 & 41.2 & $(16.6,50.6)$ & $(6.2,66.8)$ & $(1.4,80.3)$ \\
\hline$\omega(\mathrm{deg})$ & 89.0 & 63.3 & 289.7 & $(29.0,150.8)$ & $(4.2,175.8)$ & $(0.3,179.7)$ \\
\hline$\Omega(\mathrm{deg})$ & 114.7 & 41.8 & 169.8 & $(18.9,163.6)$ & $(2.5,177.6)$ & $(0.1,179.9)$ \\
\hline$T_{0}$ (years) & 2683 & 2408 & 2501 & $(2421,3736)$ & $(2122,12556)$ & $(2018,70154)$ \\
\hline$P$ (years) & 5467 & 6377 & 4279 & $(3443,12198)$ & $(2304,42183)$ & $(1673,236009)$ \\
\hline \multicolumn{7}{|c|}{ Inner orbit $\left(\min \chi^{2}=0.001, \chi^{2}\right.$ at highest probability $\left.=0.180\right)$} \\
\hline$a(\mathrm{au})$ & 7.4 & 7.9 & 4.6 & $(5.5,12.5)$ & $(4.7,27.8)$ & $(4.2,85.2)$ \\
\hline$e$ & 0.865 & 0.460 & 0.894 & $(0.568,0.969)$ & $(0.335,0.990)$ & $(0.135,0.990)$ \\
\hline$i(\mathrm{deg})$ & 97.8 & 96.1 & 111.0 & $(74.9,119.0)$ & $(28.3,155.9)$ & $(6.9,174.1)$ \\
\hline$\omega(\mathrm{deg})$ & 48.8 & 16.8 & 1.8 & $(13.7,163.9)$ & $(2.0,178.0)$ & $(0.1,179.9)$ \\
\hline$\Omega(\operatorname{deg})$ & 109.5 & 106.5 & 113.1 & $(89.5,127.8)$ & $(44.7,151.1)$ & $(2.5,177.6)$ \\
\hline$T_{0}$ (years) & 2012 & 2010 & 2012 & $(2011,2013)$ & $(2010,2053)$ & $(2010,2318)$ \\
\hline$P$ (years) & 19 & 21 & 9 & $(12,42)$ & $(9,138)$ & $(8,739)$ \\
\hline
\end{tabular}

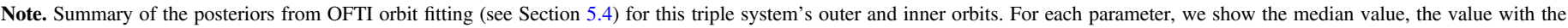
lowest $\chi^{2}\left(x_{\chi^{2}, \min }\right)$, the most likely value ( $\left.x_{\mathrm{P}, \max }\right)$, and three confidence intervals (C.I.).

Table 16

OFTI Fit Summary for Triple System WASP-12

\begin{tabular}{|c|c|c|c|c|c|c|}
\hline$x$ & Median & $x \chi^{2}, \min$ & $x_{\mathrm{P}, \max }$ & $68 \%$ C.I. & 95\% C.I. & 99.7\% C.I. \\
\hline \multicolumn{7}{|c|}{ Outer orbit $\left(\min \chi^{2}=19.894, \chi^{2}\right.$ at highest probability $\left.=20.116\right)$} \\
\hline$a(\mathrm{au})$ & 512.6 & 432.2 & 122.0 & $(322.7,990.3)$ & $(222.8,2992.2)$ & $(155.2,13293.9)$ \\
\hline$e$ & 0.531 & 0.202 & 0.858 & $(0.173,0.856)$ & $(0.025,0.980)$ & $(0.001,0.990)$ \\
\hline$i(\mathrm{deg})$ & 67.9 & 74.6 & 76.3 & $(40.1,94.8)$ & $(15.9,133.6)$ & $(3.9,167.0)$ \\
\hline$\omega(\operatorname{deg})$ & 92.4 & 141.6 & 344.8 & $(32.5,148.9)$ & $(4.7,175.4)$ & $(0.3,179.7)$ \\
\hline$\Omega(\mathrm{deg})$ & 73.8 & 69.4 & 72.8 & $(46.9,126.8)$ & $(8.3,171.7)$ & $(0.5,179.5)$ \\
\hline$T_{0}$ (years) & 5230 & 7474 & 2409 & $(3412,12532)$ & $(2609,57434)$ & $(2051,530896)$ \\
\hline$P$ (years) & 7364 & 5874 & 811 & $(3675,19784)$ & $(2102,103755)$ & $(1219,967232)$ \\
\hline \multicolumn{7}{|c|}{ Inner orbit $\left(\min \chi^{2}=0.106, \chi^{2}\right.$ at highest probability $\left.=0.600\right)$} \\
\hline$a(\mathrm{au})$ & 40.7 & 30.5 & 11.2 & $(25.8,79.3)$ & $(18.0,251.4)$ & $(12.9,1166.0)$ \\
\hline$e$ & 0.521 & 0.736 & 0.976 & $(0.169,0.853)$ & $(0.024,0.979)$ & $(0.001,0.990)$ \\
\hline$i(\mathrm{deg})$ & 77.9 & 64.7 & 84.7 & $(44.2,117.0)$ & $(17.5,153.0)$ & $(4.4,173.2)$ \\
\hline$\omega(\operatorname{deg})$ & 99.1 & 299.5 & 194.0 & $(35.9,148.4)$ & $(5.1,175.0)$ & $(0.3,179.7)$ \\
\hline$\Omega(\mathrm{deg})$ & 128.6 & 19.2 & 164.8 & $(26.5,167.9)$ & $(2.8,177.5)$ & $(0.2,179.8)$ \\
\hline$T_{0}$ (years) & 2131 & 2086 & 2034 & $(2068,2374)$ & $(2032,4005)$ & $(2011,22015)$ \\
\hline$P$ (years) & 245 & 158 & 35 & $(124,666)$ & $(72,3764)$ & $(43,37519)$ \\
\hline
\end{tabular}

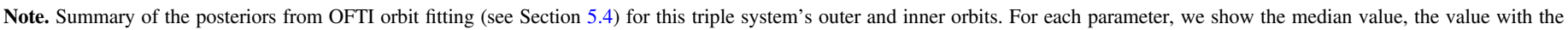
lowest $\chi^{2}\left(x_{\chi^{2}, \min }\right)$, the most likely value $\left(x_{\mathrm{P}, \max }\right)$, and three confidence intervals (C.I.). 


\section{References}

Adams, E. R., Dupree, A. K., Kulesa, C., \& McCarthy, D. 2013, AJ, 146, 9 Allen, C., Poveda, A., \& Herrera, M. A. 2000, A\&A, 356, 529

Astropy Collaboration, Robitaille, T. P., Tollerud, E. J., et al. 2013, A\&A, 558, A 33

Ballard, S., \& Johnson, J. A. 2016, ApJ, 816, 66

Baraffe, I., Chabrier, G., Allard, F., \& Hauschildt, P. H. 1998, A\&A, 412, 403

Batygin, K., Bodenheimer, P. H., \& Laughlin, G. P. 2016, ApJ, 829, 114

Bean, J. L., McArthur, B. E., Benedict, G. F., et al. 2007, AJ, 134, 749

Beaugé, C., \& Nesvorný, D. 2012, ApJ, 751, 119

Bechter, E. B., Crepp, J. R., Ngo, H., et al. 2014, ApJ, 788, 2

Becker, J. C., \& Adams, F. C. 2016, MNRAS, 455, 2980

Bergfors, C., Brandner, W., Daemgen, S., et al. 2013, MNRAS, 428, 182

Blunt, S. C., Nielsen, E., De Rosa, R. J., et al. 2017, AJ, 229 arXiv:1703.10653

Bodenheimer, P., Hubickyj, O., \& Lissauer, J. J. 2000, Icar, 143, 2

Boley, A. C., Contreras, A. P. G., \& Gladman, B. 2016, ApJL, 817, L17

Borsa, F., Scandariato, G., Rainer, M., et al. 2015, A\&A, 578, A64

Bowler, B. P. 2016, PASP, 128, 102001

Brogi, M., Snellen, I. A. G., de Kok, R. J., et al. 2012, Natur, 486, 502

Bryan, M. L., Knutson, H. A., Howard, A. W., et al. 2016, ApJ, 821, 89

Buhler, P. B., Knutson, H. A., Batygin, K., et al. 2016, ApJ, 821, 26

Butler, R. P., Wright, J. T., Marcy, G. W., et al. 2006, ApJ, 646, 505

Chatterjee, S., Ford, E. B., Matsumura, S., \& Rasio, F. A. 2008, ApJ, 686, 580

Clanton, C., \& Gaudi, B. S. 2014, ApJ, 791, 91

Collins, K. A., Kielkopf, J. F., \& Stassun, K. G. 2017, AJ, 153, 78

Cumming, A., Butler, R. P., Marcy, G. W., et al. 2008, PASP, 120, 531

Da Silva, R., Udry, S., Bouchy, F., et al. 2007, A\&A, 473, 323

Dawson, R. I., \& Chiang, E. 2014, Sci, 346, 212

Dawson, R. I., Murray-Clay, R. A., \& Johnson, J. A. 2015, ApJ, 798, 66

de Rosa, R. J., Nielsen, E. L., Blunt, S. C., et al. 2015, ApJL, 814, L3

Desidera, S., Carolo, E., Gratton, R., et al. 2011, A\&A, 533, A90

Duquennoy, A., \& Mayor, M. 1991, A\&A, 248, 485

Eastman, J. D., Beatty, T. G., Siverd, R. J., et al. 2016, AJ, 151, 45

Eggenberger, A., Udry, S., Chauvin, G., et al. 2007, A\&A, 474, 273

Eggenberger, A., Udry, S., \& Mayor, M. 2004, A\&A, 417, 353

Evans, D. F., Southworth, J., Maxted, P. F. L., et al. 2016, A\&A, 589, A58

Fabrycky, D., \& Tremaine, S. 2007, ApJ, 669, 1298

Fischer, D. A., Howard, A. W., Laughlin, G. P., et al. 2014a, in Protostars and Planets VI, ed. H. Beuther et al. (Tucson, AZ: Univ. Arizona Press), 715

Fischer, D. A., Marcy, G. W., Butler, R. P., Vogt, S. S., \& Apps, K. 1999, PASP, 111, 50

Fischer, D. A., Marcy, G. W., \& Spronck, J. F. P. 2014b, ApJS, 210, 5

Foreman-Mackey, D. 2016, The Journal of Open Source Software, 24, 1

Foreman-Mackey, D., Hogg, D. W., Lang, D., \& Goodman, J. 2013, PASP, 125,306

Fressin, F., Torres, G., Charbonneau, D., et al. 2013, ApJ, 766, 81

Ghezzi, L., Cunha, K., Smith, V. V., et al. 2010, ApJ, 720, 1290

Giguere, M. J., Fischer, D. A., Howard, A. W., et al. 2012, ApJ, 744, 4

Ginski, C., Mugrauer, M., Seeliger, M., et al. 2016, MNRAS, 457, 2173

Ginski, C., Mugrauer, M., Seeliger, M., \& Eisenbeiss, T. 2012, MNRAS, 421, 2498

Ginski, C., Mugrauer, M., Seeliger, M., \& Löhne, T. 2013, A\&A, 559, A71

Goldreich, P., \& Tremaine, S. 1980, ApJ, 241, 425

Haghighipour, N., Butler, R. P., Rivera, E. J., Henry, G. W., \& Vogt, S. S. 2012, ApJ, 756, 91

Haisch, K., Lada, E., \& Lada, C. 2001, AJ, 553, L153

Hamers, A. S. 2017, ApJL, 835, L24

Han, I., Lee, B. C., Kim, K. M., et al. 2010, A\&A, 509, A24

Hernández, J., Calvet, N., Hartmann, L., et al. 2009, ApJ, 707, 705

Howard, A. W., Johnson, J. A., W. Marcy, G., et al. 2010, ApJ, 721, 1467

Husser, T.-O., Wende-von Berg, S., Dreizler, S., et al. 2013, A\&A, 553, A6

Jofré, E., Petrucci, R., Saffe, C., et al. 2015, A\&A, 574, A50

Johansen, A., Davies, M. B., Church, R. P., \& Holmelin, V. 2012, ApJ, 758, 39

Johns-Krull, C. M., McLane, J. N., Prato, L., et al. 2016, ApJ, 826, 206

Johnson, J. A., Clanton, C., Howard, A. W., et al. 2011, ApJS, 197, 26

Johnson, J. A., Marcy, G. W., Fischer, D. A., et al. 2006, ApJ, 647, 600

Kaib, N. A., Raymond, S. N., \& Duncan, M. 2013, Natur, 493, 381

Kass, R. E., \& Raftery, A. E. 1995, J. Am. Stat. Assoc., 90, 773
Kipping, D. M. 2013, MNRAS, 434, L51

Knutson, H. A., Fulton, B. J., Montet, B. T., et al. 2014, ApJ, 785, 126

Kraus, A. L., Ireland, M. J., Hillenbrand, L. A., \& Martinache, F. 2012, ApJ, 745,19

Kraus, A. L., Ireland, M. J., Huber, D., Mann, A. W., \& Dupuy, T. J. 2016, AJ, 152,8

Lagrange, A.-M., Desort, M., Galland, F., Udry, S., \& Mayor, M. 2009, A\&A, 495,335

Latham, D. W., Bakos, G. Á, Torres, G., et al. 2009, ApJ, 704, 1107

Lin, D. N. C., Bodenheimer, P., \& Richardson, D. C. 1996, Natur, 380, 606

Lin, D. N. C., \& Papaloizou, J. 1986, ApJ, 309, 846

Lithwick, Y., \& Wu, Y. 2014, PNAS, 111, 12610

Lodieu, N., Pérez-Garrido, A., Béjar, V. J. S., et al. 2014, A\&A, 569, A120

Ma, B., Ge, J., Wolszczan, A., et al. 2016, AJ, 152, 112

Macintosh, B., Graham, J. R., Barman, T., et al. 2015, Sci, 350, 64

Mancini, L., Southworth, J., Ciceri, S., et al. 2013, A\&A, 551, A11

Mason, B. D., Hartkopf, W. I., Wycoff, G. L., et al. 2004, AJ, 128, 3012

Mason, B. D., Wycoff, G. L., Hartkopf, W. I., Douglass, G. G., \& Worley, C. E. 2001, AJ, 122, 3466

Mayer, L., Wadsley, J., Quinn, T., \& Stadel, J. 2005, MNRAS, 363, 641

Meschiari, S., Laughlin, G., Vogt, S. S., et al. 2011, ApJ, 727, 117

Montet, B. T., Bowler, B. P., Shkolnik, E. L., et al. 2015, ApJL, 813, L11

Montet, B. T., Crepp, J. R., Johnson, J. A., Howard, A. W., \& Marcy, G. W. 2014, ApJ, 781, 28

Morton, T. D., \& Winn, J. N. 2014, ApJ, 796, 47

Moutou, C., Loeillet, B., Bouchy, F., et al. 2006, A\&A, 458, 327

Moutou, C., Mayor, M., Bouchy, F., et al. 2005, A\&A, 439, 367

Moutou, C., Mayor, M., Lo Curto, G., et al. 2011, A\&A, 527, A63

Mugrauer, M., \& Ginski, C. 2015, MNRAS, 450, 3127

Mulders, G. D., Pascucci, I., \& Apai, D. 2015, ApJ, 814, 130

Naoz, S., Farr, W. M., Lithwick, Y., Rasio, F. A., \& Teyssandier, J. 2013, MNRAS, 431, 2155

Naoz, S., Farr, W. M., \& Rasio, F. A. 2012, ApJL, 754, L36

Ngo, H., Knutson, H. A., Hinkley, S., et al. 2015, ApJ, 800, 138

Ngo, H., Knutson, H. A., Hinkley, S., et al. 2016, ApJ, 827, 8

Patience, J., White, R. J., Ghez, A. M., et al. 2002, ApJ, 581, 654

Peek, K. M. G., Johnson, J. A., Fischer, D. A., et al. 2009, PASP, 121, 613

Perryman, M., Hartman, J., Bakos, G. Á, \& Lindegren, L. 2014, ApJ, 797, 14

Perryman, M. A. C., Lindegren, L., Kovalevsky, J., et al. 1997, A\&A, 323, L49

Petrovich, C. 2015, ApJ, 805, 75

Petrovich, C., \& Tremaine, S. 2016, ApJ, 829, 132

Petrovich, C., Tremaine, S., \& Rafikov, R. 2014, ApJ, 786, 101

Pichardo, B., Sparke, L. S., \& Aguilar, L. A. 2005, MNRAS, 359, 521

Piskorz, D., Knutson, H. A., Ngo, H., et al. 2015, ApJ, 814, 148

Pollack, J. B., Hubickyj, O., Bodenheimer, P., et al. 1996, Icar, 124, 62

Raghavan, D., Henry, T. J., Mason, B. D., et al. 2006, ApJ, 646, 523

Raghavan, D., McAlister, H. A., Henry, T. J., et al. 2010, ApJS, 190, 1

Rameau, J., Nielsen, E. L., Rosa, R. J. D., et al. 2016, ApJL, 822, L29

Roberts, L. C., Oppenheimer, R., Crepp, J. R., et al. 2015, AJ, 150, 103

Roberts, L. C., Turner, N. H., ten Brummelaar, T. A., Mason, B. D., \& Hartkopf, W. I. 2011, AJ, 142, 175

Sahlmann, J., Ségransan, D., Queloz, D., et al. 2011, A\&A, 525, A95

Santos, N. C., Israelian, G., \& Mayor, M. 2004, A\&A, 415, 1153

Santos, N. C., Mayor, M., Naef, D., et al. 2002, A\&A, 392, 215

Santos, N. C., Sousa, S. G., Mortier, A., et al. 2013, A\&A, 556, A150

Schlaufman, K. C., \& Winn, J. N. 2016, ApJ, 825, 62

Service, M., Lu, J. R., Campbell, R., et al. 2016, PASP, 128, 095004

Sousa, S. G., Santos, N. C., Mortier, A., et al. 2015, A\&A, 576, A94

Southworth, J. 2012, MNRAS, 426, 1291

Spalding, C., \& Batygin, K. 2016, ApJ, 830, 5

Storch, N. I., Anderson, K. R., \& Lai, D. 2014, Sci, 345, 1317

Takeda, G., Ford, E. B., Sills, A., et al. 2007, ApJS, 168, 297

Tanaka, H., Takeuchi, T., \& Ward, W. R. 2002, ApJ, 565, 1257

Torres, G. 1999, PASP, 111, 169

Torres, G., Fischer, D. A., Sozzetti, A., et al. 2012, ApJ, 757, 161

Triaud, A. H. M. J., Lanotte, A. A., Smalley, B., \& Gillon, M. 2014, MNRAS, 444,711

Udry, S., Mayor, M., Naef, D., et al. 2000, A\&A, 356, 590

van Leeuwen, F. 2007, A\&A, 474, 653

Wagner, K., Apai, D., Kasper, M., et al. 2016, Sci, 353, 673

Wang, J., Fischer, D. A., Barclay, T., et al. 2015a, ApJ, 815, 127 
Wang, J., Fischer, D. A., Xie, J.-W., \& Ciardi, D. R. 2014, ApJ, 791, 111

Wang, J., Fischer, D. A., Xie, J.-W., \& Ciardi, D. R. 2015b, ApJ, 806, 248

Wittenmyer, R. A., Horner, J., Tuomi, M., et al. 2012, ApJ, 753, 169

Wittenmyer, R. A., Wang, S., Horner, J., et al. 2013, ApJS, 208, 2

Wittrock, J. M., Kane, S. R., Horch, E. P., et al. 2016, AJ, 152, 149

Wizinowich, P. 2013, PASP, 125, 798

Wöllert, M., \& Brandner, W. 2015, A\&A, 579, A129
Wöllert, M., Brandner, W., Bergfors, C., \& Henning, T. 2015, A\&A, 575, A23 Wu, Y., \& Lithwick, Y. 2011, ApJ, 735, 109

Wu, Y., \& Murray, N. 2003, ApJ, 589, 605

Yelda, S., Lu, J. R., Ghez, A. M., et al. 2010, ApJ, 725, 331

Zucker, S., Mazeh, T., Santos, N. C., Udry, S., \& Mayor, M. 2004, A\&A, 426, 695

Zuckerman, B. 2014, ApJL, 791, L27 